\title{
WestVirginiaUniversity
}

THE RESEARCH REPOSITORY @ WVU

Graduate Theses, Dissertations, and Problem Reports

2020

\section{Neuromechanical Tuning for Arm Motor Control}

Russell Lee Hardesty Jr

West Virginia University, rlhardesty@mix.wvu.edu

Follow this and additional works at: https://researchrepository.wvu.edu/etd

Part of the Biomechanics Commons, Computational Neuroscience Commons, Motor Control

Commons, and the Systems Neuroscience Commons

\section{Recommended Citation}

Hardesty, Russell Lee Jr, "Neuromechanical Tuning for Arm Motor Control" (2020). Graduate Theses, Dissertations, and Problem Reports. 7984.

https://researchrepository.wvu.edu/etd/7984

This Dissertation is protected by copyright and/or related rights. It has been brought to you by the The Research Repository @ WVU with permission from the rights-holder(s). You are free to use this Dissertation in any way that is permitted by the copyright and related rights legislation that applies to your use. For other uses you must obtain permission from the rights-holder(s) directly, unless additional rights are indicated by a Creative Commons license in the record and/ or on the work itself. This Dissertation has been accepted for inclusion in WVU Graduate Theses, Dissertations, and Problem Reports collection by an authorized administrator of The Research Repository @ WVU.

For more information, please contact researchrepository@mail.wvu.edu. 
Graduate Theses, Dissertations, and Problem Reports

2020

Neuromechanical Tuning for Arm Motor Control

Russell Lee Hardesty Jr

Follow this and additional works at: https://researchrepository.wvu.edu/etd

Part of the Biomechanics Commons, Computational Neuroscience Commons, Motor Control Commons, and the Systems Neuroscience Commons 


\title{
Neuromechanical Tuning for Arm Motor Control
}

\author{
Russell Hardesty
}

Dissertation submitted to the School of Medicine at West Virginia University

In partial fulfillment of the requirements for the degree of

Doctor of Philosophy in Neuroscience

\author{
Valeriya Gritsenko, PhD, Chair \\ Sergiy Yakovenko, PhD \\ Jennifer Collinger, $\mathrm{PhD}$ \\ Nicholas Brandmeir, MD \\ Kostantinos Sierros, PhD
}

Neuroscience Graduate Program

Morgantown, WV

2020

Keywords: computational neuroscience, motor control, musculoskeletal modeling, neuromechanics 


\title{
Abstract \\ Neuromechanical Turning in Arm Motor Control
}

\author{
Russell Hardesty
}

Movement is a fundamental behavior that allows us to interact with the external world. Its importance to human health is most evident when it becomes impaired due to disease or injury. Physical and occupational rehabilitation remains the most common treatment for these types of disorders. Although therapeutic interventions may improve motor function, residual deficits are common for many pathologies, such as stroke. The development of novel therapeutics is dependent upon a better understanding of the underlying mechanisms that govern movement.

Movement of the human body adheres to the principles of classic Newtonian mechanics. However, due to the inherent complexity of the body and the highly variable repertoire of environmental contexts in which it operates, the musculoskeletal system presents a challenging control problem and the onus is on the central nervous system to reliably solve this problem. The neural motor system is comprised of numerous efferent and afferent pathways with a hierarchical organization which create a complex arrangement of feedforward and feedback circuits. However, the strategy that the neural motor system employs to reliably control these complex mechanics is still unknown.

This dissertation will investigate the neural control of mechanics employing a "bottom-up" approach. It is organized into three research chapters with an additional introductory chapter and a chapter addressing final conclusions. Chapter 1 provides a brief description of the anatomical and physiological principles of the human motor system and the challenges and strategies that may be employed to control it. Chapter 2 describes a computational study where we developed a musculoskeletal model of the upper limb to investigate the complex mechanical interactions due to muscle geometry. Muscle lengths and moment arms contribute to force and torque generation, but the inherent redundancy of these actuators create a high-dimensional control problem. By characterizing these relationships, we found mechanical coupling of muscle lengths which the nervous system could exploit. Chapter 3 describes a study of muscle spindle contribution to muscle coactivation using a computational model of primary afferent activity. We investigated whether these afferents could contribute to motoneuron recruitment during voluntary reaching tasks in humans and found that afferent activity was orthogonal to that of muscle activity. Chapter 4 describes a study of the role of the descending corticospinal tract in the compensation of limb dynamics during arm reaching movements. We found evidence that corticospinal excitability is modulated in proportion to muscle activity and that the coefficients of proportionality vary in the course of these movements. Finally, further questions and future directions for this work are discussed in the Chapter 5. 


\section{Acknowledgements}

I would like to thank and acknowledge the many individuals who have helped me in my graduate career.

First, I would like to thank my advisor, Dr. Valeriya Gritsenko for the guidance and support she has provided me. Her patience and commitment to both my graduate education and development as a scientist has enabled me to grow tremendously over the last few years. Under her tutelage, I have learned vital skills including, but not limited to, experimental design, scientific rigor, critical thinking, and scientific communication.

I would like to thank all my committee members: Dr. Sergiy Yakovenko, Dr. Jennifer Collinger, Dr. Nicholas Brandmeir, and Dr. Kostantinos Sierros. Their invaluable input and feedback greatly shaped both my dissertation work and my individual development.

I would also like to thank my fiancé, Abigail Myers. Her unending support and compassion remain the greatest gifts that graduate school has given me.

Thank you to my laboratory colleagues, specifically Dr. Matthew Boots, Trevor Moon, Dr. Anton Sobinov, Brad Pollard, Lisa Kogan, Ariel Thomas, Dr. Erienne Olesh, Neha Lal, Sarah Freeman, and all others. You have all been outstanding colleagues but more importantly you have been and continue to be outstanding people and friends.

Thank you to our collaborators, specifically the RNEL laboratory at the University of Pittsburgh, as well as all of our funding sources. Thank you also to the West Virginia University Graduate program, in particular our Neuroscience program directors: Dr. Richard Dey, Dr. Bernard Schruers, and Dr. Randy Nelson.

Finally, a very special thank you to all of my family and friends who have supported me through this endeavor. I would not have been able to purse my Ph.D without you. In particular, thank you to my mother who, even though she did not live to see it, instilled the necessary work ethic and stubbornness that enabled me to pursue my career ambitions. 


\section{Table of Contents}

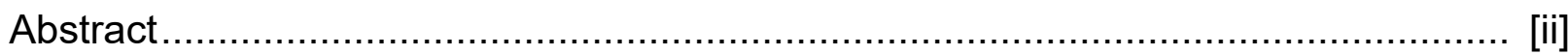



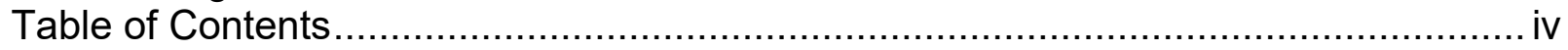

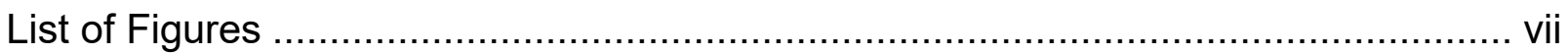

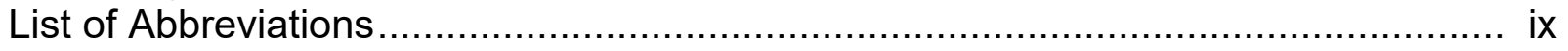

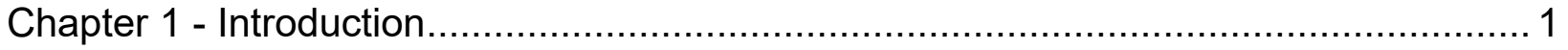

The Social and Clinical Relevance of Movement ...................................................... 1

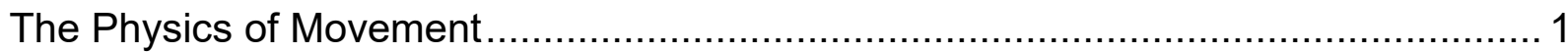

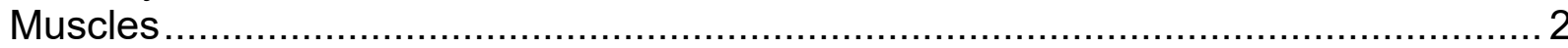



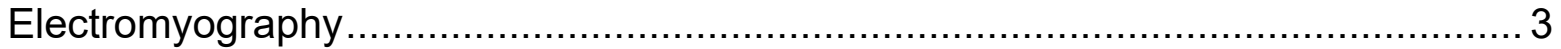

Mathematical Descriptions of Muscle Force ...................................................... 4

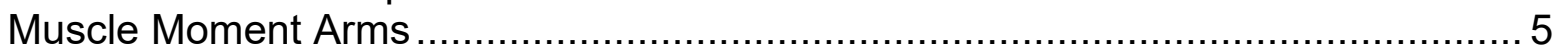

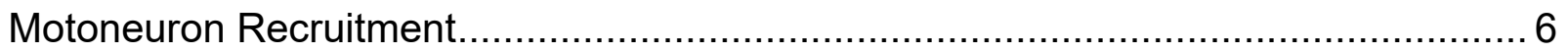

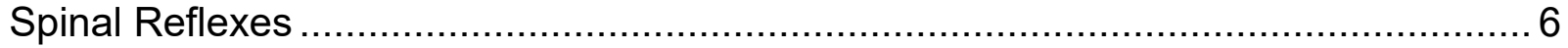

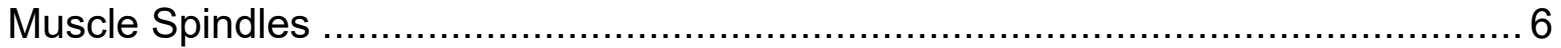

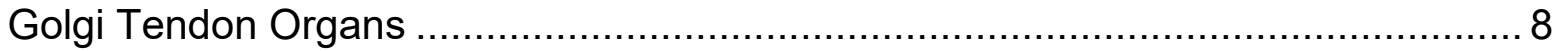





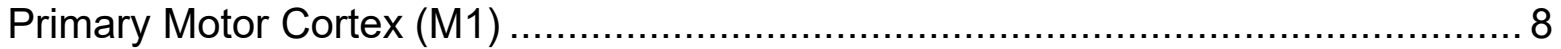

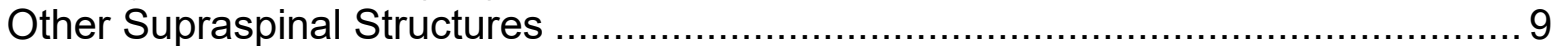

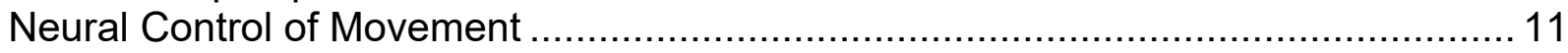

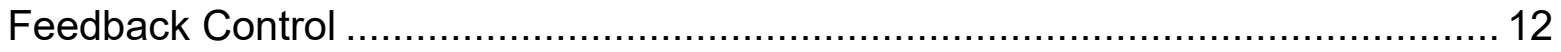

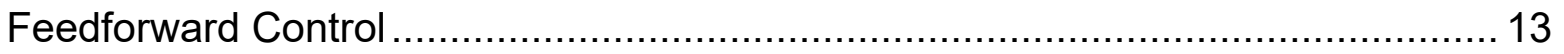

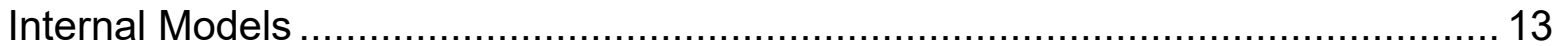

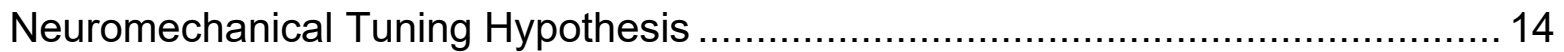

Dissertation Summary ........................................................................... 14

Chapter 2 - Biomechanical constraints underlying motor primitives derived from the

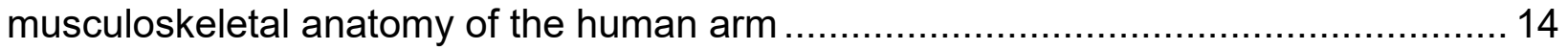

Abstract 


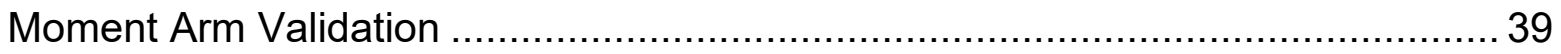

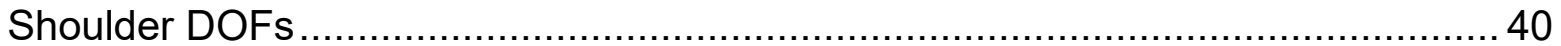

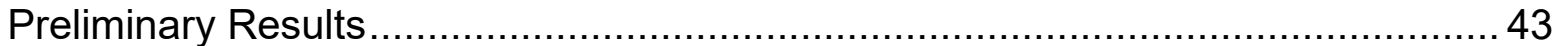

Chapter 3 - Computational evidence for nonlinear feedforward modulation of fusimotor

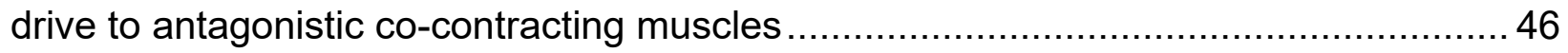

Abstract

Chapter 4 - Corticospinal Excitability is Modulated During Dynamically-distinct

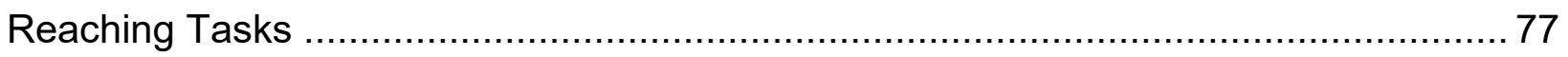

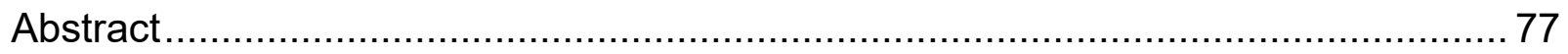

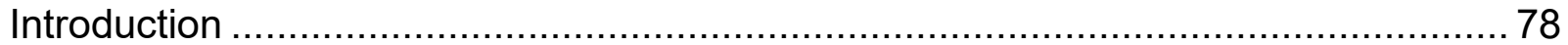

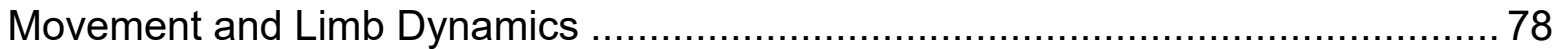

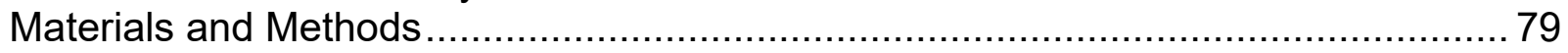

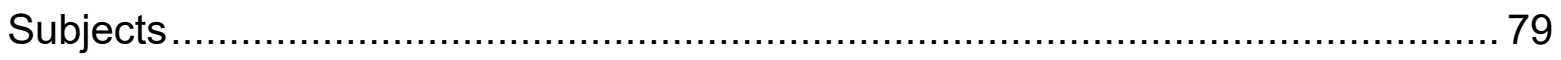

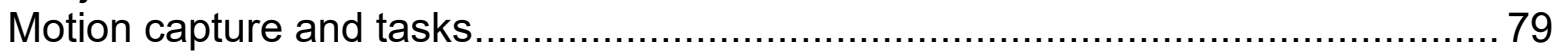

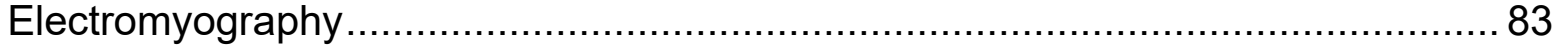

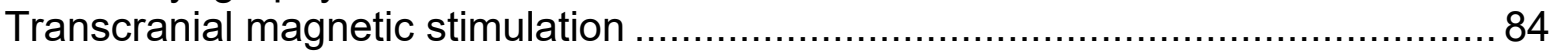

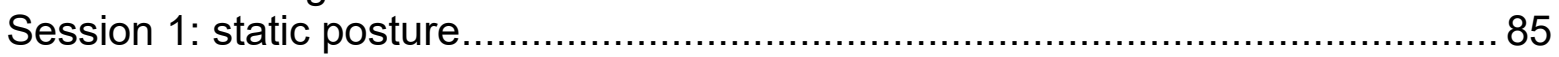

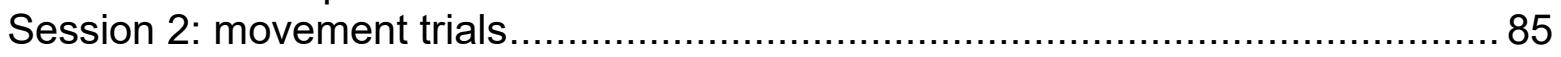







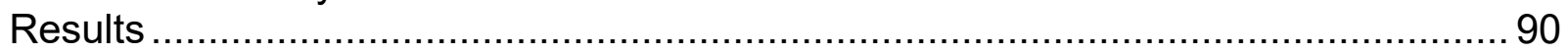

Corticospinal Excitability During Posture Maintenance ....................................... 90

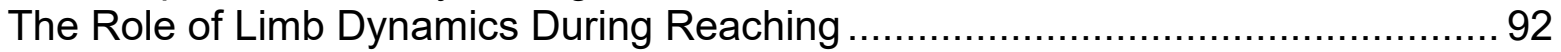

Corticospinal Excitability During Movement.................................................... 94

The Role of Limb Dynamics in Corticospinal Excitability ........................................ 98

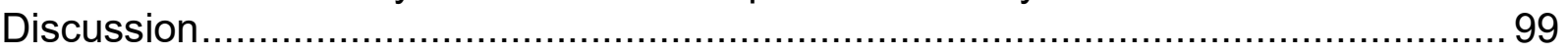

Chapter 5 - Discussion and Future Directions ....................................................... 101

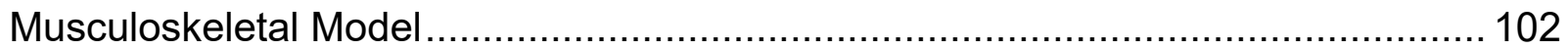

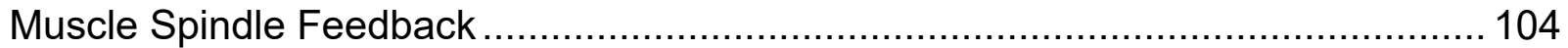

Descending Corticospinal Contribution .......................................................... 105 


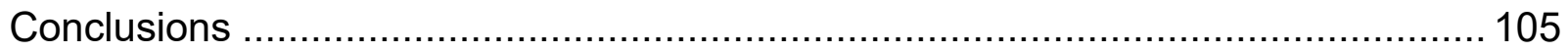

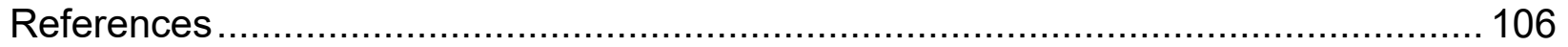




\section{List of Figures}

Figure 1-1: Schematic of HZL muscle model. ......................................................... 5

Figure 1-2: Hierarchical organization of motor system ........................................... 10

Figure 2-1: Illustration of the model and local coordinate systems............................ 18

Figure 2-2: The difference between $\mathrm{r} 2$ values for the correlations between muscle

lengths as a function of the number of selected postures ......................................... 20

Figure 2-3: Examples of muscle lengths for the pronator teres, a single 2-DOF muscle originating on the humerus and attaching on the radius, in two subjects .................... 24

Figure 2-4: Examples of the correlations between muscle lengths in a single subject .. 27

Figure 2-5: Hierarchical clustering methodology and examples for two subjects .......... 28

Figure 2-6: Reliability of clustering across subjects............................................... 30

Figure 2-7: Mean hierarchical clustering across all subjects ..................................... 31

Figure 2-8: Example of changes in moment arms as a function of posture .................. 40

Figure 2-9: Coordinates of the shoulder joint. .................................................... 41

Figure 2-10: Example of moment arm validation............................................... 42

Figure 2-11: Qualitative assessment of moment arm quality.................................. 43

Figure 2-12: Hierarchical clustering of shoulder muscle moment arms...................... 45

Figure 3-1: Illustrations of the experimental setup and arm models. .......................... 51

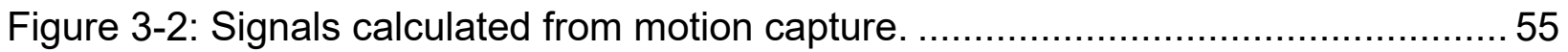

Figure 3-3: Normalized EMG, co-contraction, and la profiles for muscles spanning the shoulder.

Figure 3-4: Normalized EMG, co-contraction, and la profiles for muscles spanning the elbow and wrist. 65

Figure 3-5: The relationships between EMG and la profiles per task in a representative individual. 67

Figure 3-6: Comparison between muscle length and la clustering. 68

Figure 3-7: The consistency of hierarchical clustering between EMG and la within and

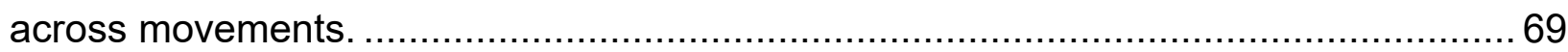

Figure 3-8: Fusimotor-based changes in the clustering of la profiles. ........................ 72

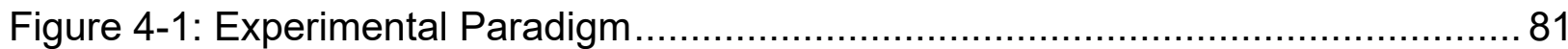






Figure 4-3: Postural corticospinal excitability in BicL............................................... 91

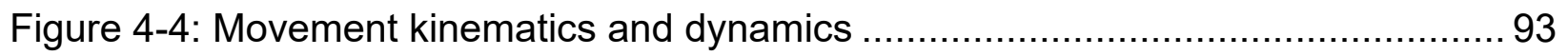

Figure 4-5: BicL \& TriLo corticospinal excitability during movement ............................ 94

Figure 4-6: BicL MEP variance explained by EMG ................................................. 96

Figure 4-7: Multi-muscle MEP magnitude and gain................................................ 97 


\section{List of Abbreviations}

A.U.: $\quad$ Arbitrary Units

B: $\quad$ Fowlkes-Mallow Index

BIC: $\quad$ Bayesian Information Criterion

CNS: Central Nervous System

CPG: $\quad$ Central Pattern Generator

DOF: Degree of Freedom

ECoG: Electrocorticography

EEG: $\quad$ Electroencephalography

EMG: $\quad$ Electromyography

F: $\quad$ Force

GTO: $\quad$ Golgi Tendon Organ

HVE: Heterogeneous Variance Explained

HZL: He-Zajac-Levine

IN: Interneuron

IRB: Institutional Review Board

$\mathrm{L}: \quad$ Length

LED: $\quad$ Light Emitting Diode

M1: $\quad$ Primary Motor Cortex

MEP: $\quad$ Motor Evoked Potential

MUAP: $\quad$ Motor Unit Action Potential

NNMF: Non-negative Matrix Factorization

PCA: $\quad$ Principal Component Analysis

pM: $\quad$ Premotor cortex

r: Pearson correlation coefficient

R: Moment Arm

RMS: $\quad$ Root Mean Square 
RMT: $\quad$ Resting Motor Threshold

RT: $\quad$ Reaction Time

SD: $\quad$ Standard Deviation

TMS: Transcranial Magnetic Stimulation

V: $\quad$ Velocity

VR: $\quad$ Virtual Reality 


\section{Chapter 1 - Introduction \\ The Social and Clinical Relevance of Movement}

Movement is among the most fundamental of human behaviors. It provides the means to interact with our environment and to execute our decisions. It should be no surprise then that a properly functioning motor system is critical to an individual's quality of life. This

observation is perhaps most evident in circumstances where the motor system has become impaired, such as after a stroke or spinal cord injury. Indeed, previous studies have shown that motor deficits have a detrimental effect on numerous metrics of quality of life, including physical, economic, social, sexual, and psychological circumstances (Cholewa et al., 2017; Lo Buono et al., 2017; Martinez-Martin, 2017; Wit et al., 2017; Duzgun Celik et al., 2018; Ramos-Lima et al., 2018; Gendre et al., 2019). Physical and occupational rehabilitation remains the primary treatment for these disorders and while these methods can improve motor function, residual deficits are common (Kelly et al., 2018). Furthermore, motor deficits result in significant societal costs. The indirect costs of stroke alone are estimated to be $\$ 126.4$ billion from 2012 to 2013 (Benjamin et al., 2017). Because of these high costs, both to the individual and the society, there is a need for novel treatments of these disorders. Importantly-, a better understanding of the underlying mechanisms that govern movement are necessary to accomplish this aim.

\section{The Physics of Movement}

At its core, human movement can be defined as a classical mechanics problem. The human body is comprised of over 600 bony segments joined together by over 200 diverse joints with each joint having one or more rotational degrees of freedom (DOF). The motion of a specific segment can therefore be described by the second Newtonian law of rotational motion (Newton, 1713):

$$
\sum \vec{\tau}=I \vec{\alpha}
$$

Here, $\mathrm{T}$ is net torque and $\alpha$ is the resulting angular acceleration of the segment with I inertia . Defined by this law, an object experiencing no net force or torque will be at static equilibrium, while objects experiencing some non-zero torque will accelerate. Therefore, 
movement results from all active forces applied by muscle actuators (see Muscle Anatomy and Force Generation), external passive forces (e.g., gravity), and other passive torques (e.g. inertia) (Olesh et al., 2017a). These torques summate to accelerate a limb and generate movement.

$$
\sum \vec{\tau}=\overrightarrow{\tau_{M}}+\overrightarrow{\tau_{P}}
$$

Here, TM is torque due to muscle contraction and TP is torque generated passively due to external forces and the inertia of the limb. Despite the universal robustness of this mathematical description, human movement is complicated by a high number of DOFs and the variety of forces that may act upon a limb. Due to this complexity, there exists an infinite number of kinematic arrangements which will result in the same desired endpoint. This apparent redundancy is referred to as the "motor redundancy problem", first coined by Nikolai Bernstein (Bernstein, 1967). Furthermore, the environmental context of a movement creates external and passive torques that act upon a limb (e.g. the lower limb will experience different forces running on a sandy beach versus running on rigid pavement). Therefore, generating the same kinematic trajectory in a different environmental context always require distinct muscle forces/torques. Finally, one must consider the mechanical variability that would exist between individual persons due to different body sizes and compositions, such as differences in segment mass, the distribution of mass per segment, or inter-joint distances. Additional variability arises from anatomical differences in muscle paths and force production. This high degree of complexity and apparent redundancy creates a difficult control problem that the nervous system must solve.

\section{Muscles}

\section{Muscle Anatomy and Force Generation}

While various forces may act upon a limb, it is muscles that are the physiological actuators. Muscle force production is inherently determined by the underlying physiology and anatomy of muscle tissue. Skeletal muscle is comprised of parallel single-celled muscle fibers which, in turn, consist of tubular myofibrils. These myofibrils have repeating sections called sarcomeres containing overlapping thick and thin filaments - consisting 
of myosin and actin respectively - which form cross-bridges via the myosin heads. Multiple muscle fibers are innervated by a single a-motoneuron originating from the ventral horn of the spinal cord forming a motor unit. Action potentials propagating along the a-motoneuron axons depolarize the presynaptic membrane of the axon terminal and release acetylcholine into the neuromuscular junction opening $\mathrm{Na}^{+}$ion channels on the postsynaptic muscle fiber membrane (motor end plate). As $\mathrm{Na}^{+}$ions enter the cell via ionic diffusion, the muscle fiber depolarizes generating a motor unit action potential (MUAP). The MUAP propagates to the T-tubules which penetrate into the cell resulting in the opening of voltage-gated $\mathrm{Ca}^{2+}$ channels. As the intracellular $\mathrm{Ca}^{2+}$ concentration increases, it binds to the myosin heads causing a conformational change which shortens the sarcomere length (i.e. muscle contraction) and generates force. This process, called the "power stroke" is repeatedly cycled to maintain contractile force over time. These intracellular dynamics are important in describing two characteristics of muscle force generation: the force-length and force-velocity relationships.

Skeletal muscle generates force in a length-dependent manner, termed the force-length relationship. The resulting force generated by muscle contraction is proportional to the overlapping of the thick and thin filaments. At the extremes of the sarcomere length, there is less overlapping, resulting is less cross-bridging and less subsequent force generation. This relationship results in a nonlinear force-length dependency; force generation is largest at an optimal length where cross-bridging is at its highest proportion and decreases at lengths above or below this optimum. Furthermore, force generation is also dependent upon the velocity of contraction. The temporal dynamics of the contraction cycle (myosin binding, $\mathrm{Ca}^{2+}$-mediated phosphorylation, conformational changes, and dephosphorylation) rate-limit the efficiency of force generation. At higher velocities, a smaller proportion of myosin heads will be actively bound to the actin filaments and dephosphorylated at a given time step. Therefore, muscle force decreases as a product of contractile velocity during concentric contractions.

\section{Electromyography}

The synchronous firing of motor units generates a myoelectric potential that can be measured using dipolar electrodes either inserted into the muscles (intramuscular) or 
placed superficially on the skin (surface). This technique, called electromyography or EMG, can record electrical potentials generated by propagating MUAPs which can be interpreted as a proxy for muscle activation. To achieve this aim, EMG signals are commonly rectified to address the polarity due to electrode placement and then filtered to approximate an "envelope" of muscle activity (De Luca et al., 2010). This technique provides a means to experimentally measure the end-effector of the neural motor system, motoneuron activity.

\section{Mathematical Descriptions of Muscle Force}

The absolute magnitude of force that a given muscle generates is a product of the gross muscle architecture and underlying physiology. Several mathematical models have been proposed which aim to generalize force production in terms of specific physiological parameters, e.g. optimal fiber length or passive elasticity. Winters and Stark broadly classified these models into one of three categories, 1) simple $2^{\text {nd }}$ order systems, 2) Hillbased Lumped Parameter Models, and 3) Huxley-Based Distributed-Parameter Models (Winters and Stark, 1987). For brevity, this manuscript will introduce the Hill-type muscle model, with the He-Zajac-Levine (HZL) model as it is widely used and the basis of the data presented in chapter 2.

The Hill-type muscle model describes the transformation of muscle activation to force (Zajac, 1989; He et al., 1991). Motoneuron discharge triggers the release of calcium into the sarcoplasmic reticulum (see Muscle Anatomy and Force Generation) which results in an intermediate state of "active muscle" as described by Hill (Hill, 1938). The dynamics of this transformation, neural excitation $(u)$ to muscle activation $(a)$, is termed excitationcontraction coupling and can be modeled as a simple first-order equation,

$$
\frac{d a(t)}{d t}+\left[\frac{1}{\tau_{a c t}} \cdot(\beta+[1-\beta] u(t))\right] \cdot a(t)=\left(\frac{1}{\tau_{a c t}}\right) \cdot u(t)
$$

where $\beta=\frac{\tau_{\text {act }}}{\tau_{\text {deact }}}$, and $\tau_{\text {act }}$ and $\tau_{\text {deact }}$ are rate constants for muscle activation and deactivation, respectively (Zajac, 1989).

Once muscle fibers enter this activated state, the transformation of muscle activation to contractile force is governed by the mechanical properties of muscle, tendons, and 
connective tissues. The Hill-type muscle model approximates the contraction dynamics due to these mechanical properties (Zajac, 1989). It generally consists of a contractile element, which describes the non-linear transformation of activation to force including the force-length and force-velocity relationships, and a parallel passive element, which describes the viscoelastic properties of muscles (Figure 1-1).

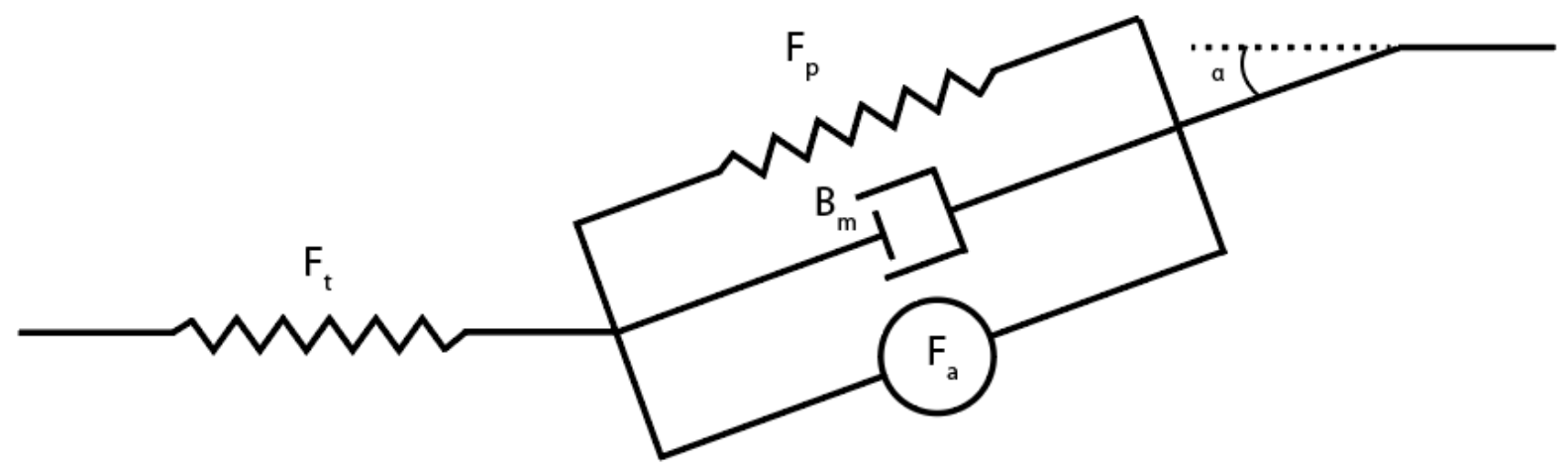

Figure 1-1: Schematic of Hill-type muscle model.

Ft is the passive force due to tendon compliance; $\mathrm{Fp}$ is passive force due to passive tissues inside the muscle, i.e. aponeurosis, membranes, etc; Fa is active force of the contractile element; alpha is pennation angle; is a viscous component due to force velocity relationship

Muscle force is transferred to bony segments via tendons and the mechanical compliance of tendons contribute to the dynamics of force production. An additional passive component may be included in series which accounts for tendon compliance (He et al., 1991). The pairing of excitation-contraction coupling with the Hill-type muscle model provides a mathematical approximation which relates neural excitation to muscle force production.

\section{Muscle Moment Arms}

Movement results from the net summation of torques around a DOF. While the nervous system can directly control the force production of muscles, the torque generated by each muscle is also a product of the distance from that muscle to the center of rotation of each joint that it spans, termed the moment arm (R). Muscle moment arms are therefore 
defined by their physiological path, which can change with the kinematic posture of a limb as muscles slide or shift after a change in posture and during movement. Boots et al. found that dynamic moment arms resulted in significantly different torque production than constant moment arms (Boots et al., 2020). It would logically follow that the nervous system would therefore need to consider the posture-dependent states of moment arms

to produce appropriate muscle forces for a desired movement. Whether dynamic moment arm compensation is the result of a feed-forward approximation, such as an internal model, or sensorimotor feedback is not well understood.

\section{Motoneuron Recruitment}

Muscle contraction is the product of neural discharges from motoneurons located in the ventral horn of the spinal cord which each innervate one or more muscle fibers, termed a motor unit. These motor units are recruited by the nervous system to generate muscle force and subsequent movement. To maintain muscle force production beyond the short time scales of neural action potentials, multiple motor units must be sequentially recruited.. Smaller motoneurons innervate smaller slow-twitch fatigue-resistant fibers, which produce lower forces, while larger motoneurons innervate larger fast-twitch muscles, which generate larger forces but fatigue quicker. As force production increases, motoneurons are recruited in a specific order according to their size where smaller motoneurons are recruited first followed by those innervating fast-twitch/fatigue resistant muscle fibers, and finally fast-twitch/fatigable muscle fibers, termed Henneman's size principle.

\section{Spinal Reflexes}

\section{Muscle Spindles}

In addition to motoneurons, the spinal cord contains significant neural circuitry that can modulate the likelihood of motoneuron action potentials. Amongst the most widely studied are those termed spinal reflexes, which are mediated by afferent pathways in the spinal cord that can synapse directly onto motoneurons. One such pathway originates in muscle spindles. Muscle spindles are mechanotransducers consisting of intrafusal muscle fibers attached to the force-producing extrafusal fibers of a muscle. These structures also 
consist of a primary afferent sensory neuron (la) which project via the dorsal horn onto spinal motoneurons and interneurons in the spinal cord. These afferent projections can form 1) excitatory monosynaptic connections onto motoneurons of the homonymous muscle, 2) excitatory monosynaptic connections onto motoneurons of agonist muscles, or 3) inhibitory connections on motoneurons of antagonist muscles via an interneuron. When a muscle lengthens, the primary afferent is excited resulting in the recruitment of the homonymous muscle and its agonists while the antagonists are inhibited. This structural-functional relationship creates a negative-length feedback loop which can quickly respond to mechanical perturbations. The resulting behavior is referred to as the stretch-reflex, where a sudden lengthening of a muscle causes an immediate contraction of that muscle in response. A recent study has suggested that muscle spindles may also encode characteristics of force, particularly the transient change in muscle force at the onset of muscle lengthening (Blum et al., 2017). Whether muscle spindles simply act as length sensors or they encode a more complex sensory signal, this pathway provides a mechanism to provide sensory information about the state of a muscle back to the nervous system as first hypothesized by Charles Sherrington Indeed muscle spindle afferents project not only to spinal motoneurons but also to spinal interneurons which then have projections to the somatosensory cortex and the cerebellum (Liddell and Sherrington, 1924).

Muscle spindles contain primary (la) afferents and secondary (II) afferents which respond primarily to either changes in the rate of change of muscle length (velocity) or simply changes in muscle length, respectively. Matthews 1959 showed that muscle spindle sensitivity could be modulated in two distinct manners: 1) the afferent sensitivity to changes in length magnitude (static) or 2 ) the sensitivity to changes in muscle length velocity (dynamic) (Matthews, 1959a). This modulation of sensitivity is driven by $\beta$ - and $\gamma$ - motor neurons which innervate the intrafusal muscle fibers of the muscle spindle, called fusimotor neurons. This fusimotor input keeps the intrafusal fibers taut across the range of muscle lengths. However, the role of fusimotor neurons during movement is still poorly understood due, in part, to the difficulty in directly recording from these neurons in moving animals. 


\section{Golgi Tendon Organs}

In addition to muscle spindles, another mechanotransducer of note is the Golgi tendon organs. This sensory organ is located in muscle tendons and its tree-like sensory endings become deformed when force is exerted on the tendon increasing the firing of $\mathrm{lb}$ sensory afferents. These lb afferents project via the dorsal horn onto inhibitory spinal interneurons which then synapse onto spinal motoneurons. This pathway creates a negative-force feedback loop which begins to inhibit motoneuron firing with increasing force. Interestingly, during locomotion, the effect of $\mathrm{Ib}$ afferents is reversed from inhibitory to excitatory creating positive-force feedback, which aids muscle force generation (Grey et al., 2007). However, the effect of the $\mathrm{lb}$ projections during reaching is still unknown. Furthermore, Golgi tendon organs provide sensory information about the state of muscle force and, similar to muscle spindle afferents, Ib afferents have downstream projections to the somatosensory cortex and cerebellum.

\section{Descending Motor Input}

\section{Motor Cortex}

In addition to spinal reflexes, spinal motoneurons are excited by a number of inputs descending from supraspinal areas of the nervous system. Stimulation of regions of the frontal cortex can induce transient muscle contractions implying an anatomical and functional relationship (Penfield and Rasmussen, 1950a; Graziano et al., 2002). Singlecell, electrocorticographic (ECoG), and electroencephalographic (EEG) recordings during movement show a marked increase in neuronal activity in these same regions. Finally, lesions to these areas, either observed clinically or in reduced experimental preparations, lead to severe motor deficits or paralysis(Hoffman and Strick, 1995; Ghika-Schmid et al., 1997).

\section{Primary Motor Cortex (M1)}

The primary motor cortex is located at the precentral gyrus and is widely considered responsible for controlling voluntary movement. It has a somatotopic organization with direct and indirect projections to spinal motoneurons (Leyton and Sherrington, 1917; Lawrence and Kuypers, 1968; Lesser et al., 1998). While its importance in voluntary movement is well established from stimulation and lesion experiments, the precise role 
that it plays in the broader control strategy of the motor system is still a point of controversy (Lawrence and Kuypers, 1968). Single-cell recordings in the primary motor cortex have correlated neuronal firing rates with physical characteristics of movement such as endpoint direction (Georgopoulos et al., 1986), velocity (Moran and Schwartz, 1999), and muscle force (Evarts, 1968). These pyramidal neurons project both to the brainstem and the spinal cord with a small portion synapsing directly onto spinal motoneurons via the corticospinal tract in humans.

\section{Other Supraspinal Structures}

In addition to the cortex, there are a number of other regions in the CNS which contribute to the descending motor pathways. As these are not the focus of the work presented in this dissertation, they will only be briefly introduced. For example, the reticular formation which projects to spinal motoneurons via the reticulospinal tract. This descending input is associated primarily with proximal muscles involved in maintaining posture and balance. Lesions in the reticulospinal tract in macques show an impaired ability to maintain upright posture (Lawrence and Kuypers, 1968). Furthermore, reticulospinal neuronal activity is increased with both timings and magnitudes which correspond to periods of postural adjustments in walking cats (Prentice and Drew, 2001). In addition to the reticular formation, the vestibulospinal tract originates in the vestibular nucleus in the brainstem. The vestibular nucleus integrates sensory input from the semi-circular canals and otolith organs and projects to spinal motoneurons. Similar to the reticulospinal tract, the vestibulospinal tract is primarily associated with maintaining posture, interlimb coordination and balance. Furthermore, the cerebellum receives numerous sensory afferents from the spinal cord and projects to several brainstem nuclei and the cortex. The cerebellum has been shown to be critical for both movement error reduction and motor learning (Kawato et al., 1987; Bastian et al., 1996; Wolpert et al., 1998). Finally, the basal ganglia plays an important role in movement initiation which can clinically observed in the symptoms of Parkinson's patients in the form of hypokinesia (Wichmann et al., 2017). All of these structure, and those previously described, form a complex network of efferent and afferent projections which ultimately integrate at the final common pathway of spinal motoneurons (Figure 1-1). 


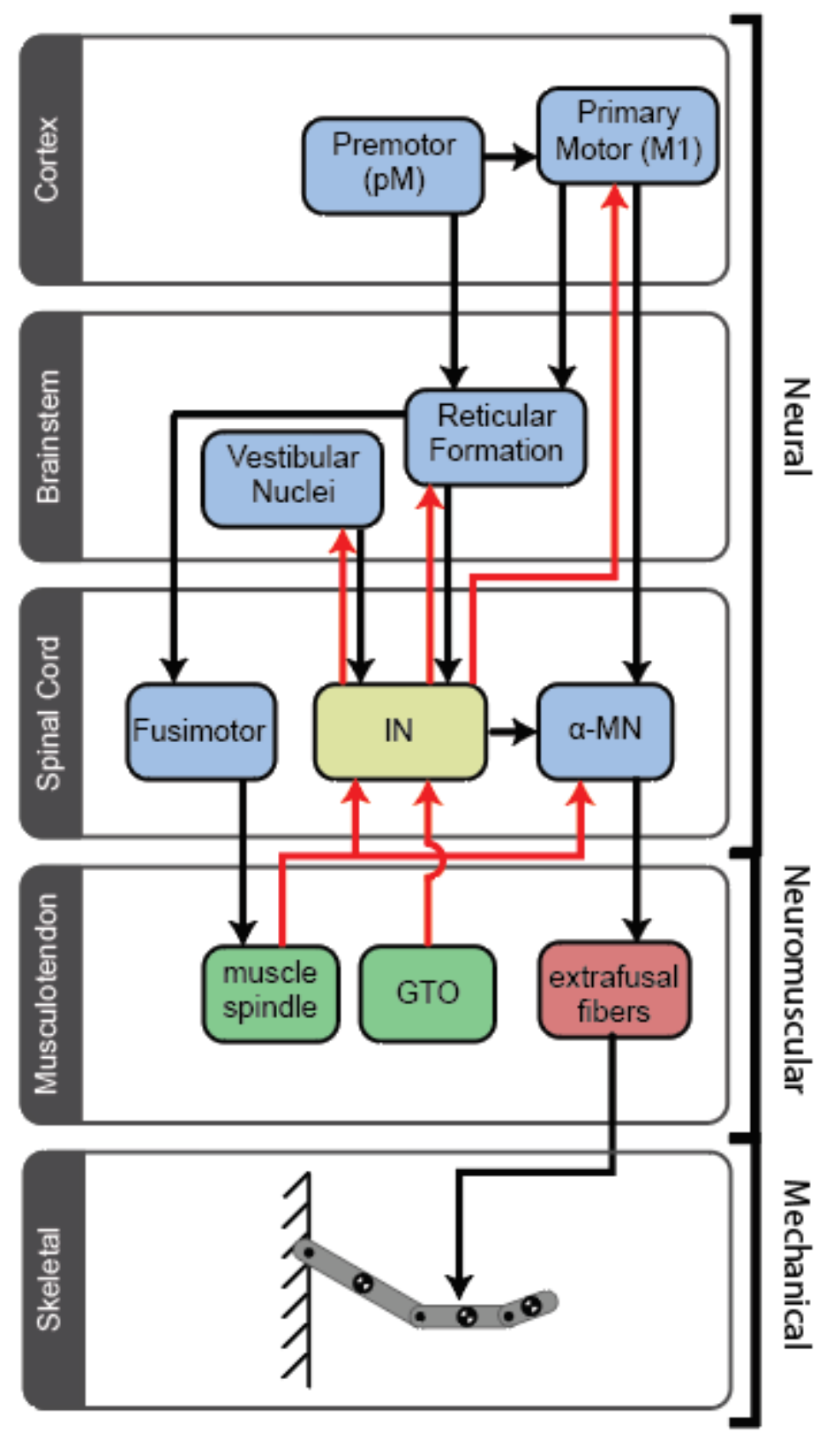

Figure 1-2: Hierarchical organization of motor system

Partial schematic of the efferent (black) and afferent (red) projections within neural motor system. It is important to note that this schematic is not comprehensive of the entire motor system (e.g. it does not include the cerebellum or basal ganglia). 


\section{Neural Control of Movement}

Despite the mechanical complexity and inherent variability of human motion, there are characteristics of movement which seem to be well conserved across individuals. For example, during arm reaching tasks the limb endpoint consistently travels along a linear trajectory between two target locations, which has been observed both in primate and human studies. Additionally, the endpoint velocity consistently follows a bell-shaped curve, where the velocity peak occurs halfway through an intended movement (Hollerbach and Flash, 1982; Dekleva et al., 2018). These invariant characteristics are maintained even when the external or passive torques acting on the limb differ between target locations. These findings suggest that the nervous system must have some means of compensating/controlling for these extrinsic and passive torques (Crevecoeur et al., 2009). Furthermore, if the dynamics of a movement is altered - such as by the application of an external force field - participants will quickly adapt to these novel dynamics, restoring the linear trajectory (Giszter et al., 1993; Shadmehr and Mussa Ivaldi, 1994; Rancourt and Hogan, 2001; Franklin et al., 2003; Pasalar et al., 2006; Leclere et al., 2019). This observation further suggests that the nervous system actively shapes these characteristics of movement and that they are not just incidental occurrences. Therefore, to control the limb, the motor system must generate muscle forces that either compensate or take advantage of these extrinsic dynamics.

To generate appropriate muscle forces for an intended movement, the nervous system must select which muscles to excite and to what extent. This selection process would necessarily require the nervous system to consider the current state of the musculoskeletal system as well as any extrinsic and intrinsic passive forces that may impact movement. This information, both intrinsic and extrinsic, is provided via the various sensory inputs such as vision and proprioception. Sensory feedback must then be integrated and transformed into motor output, i.e., muscle excitation. This transformation involves numerous areas of the central and peripheral nervous system, including the cortex, brainstem, cerebellum, basal ganglia, and spinal cord. Subsequently, the transformation from muscle excitation to kinematic movement is determined by the 
physiological and mechanical properties of muscle as well as the mechanics of the skeletal system and the external environment.

\section{Feedback Control}

Sensory feedback provides a mechanism for error correction by integrating delayed information about the ongoing movement into the motor control strategy. This contribution can most aptly be observed in the presence of error-inducing perturbations. For example, when an external force is applied to the limb that deviates an intended trajectory, sudden changes in muscle length excite compensating muscles via the la and/or lb afferent pathways. The activation of these muscles then quickly corrects the muscle length and force error. In this regard, spinal reflexes act to maintain mechanical homeostasis, correcting for any unexpected limb dynamics.

In addition to providing error correction, sensory feedback can provide a more generalized control strategy by responding to an updated desired state of the musculoskeletal system. In this view, much the same way that a mechanical perturbation may result in muscle contraction via the stretch reflex, different stimuli may evoke a new state, which in turn drives other motor responses to form more complex movements (Sherrington, 1910). For example, the presentation of food or the occurrence of an obstacle would evoke an appropriate motor response to either reach for the food or step over the obstacle. In this framework, sensory feedback drives muscle activation to match the current musculoskeletal state with that of the new desired state. The equilibrium-point hypothesis suggests such a control strategy mediated by muscle spindles (Asatryan and Feldman, 1965). This theory states that joint kinematics are the result of the tuning of agonistantagonist stretch reflex gains to create an equilibrium point $(\lambda)$ for the joint which defines its current state. To then move, a new equilibrium point is selected, and muscle contractions are generated based upon the resulting imbalance between the agonistantagonist muscle spindle feedback. One of the advantages of this hypothesis is that it provides a singular mechanism which integrates both voluntary movement and posture maintenance. 


\section{Feedforward Control}

While the motor system can respond to unexpected perturbations via feedback mechanisms such as spinal reflexes, it can also form preemptive motor commands in anticipation of expected limb dynamics (Gatev et al., 1999; Kagaya and Patek, 2016). This feedforward control strategy enables the motor system to preemptively recruit muscles to compensate for sources of joint torque not directly created by muscle contraction, e.g. passive torques (Hirashima et al., 2003). This approach may be particularly helpful for fast movements that are susceptible to destabilizing feedback delays. Another example is catching a falling object, which requires not only anticipation of the trajectory and position of the object, but also a prediction of the object's weight and the force that it may exert on the limb (Lacquaniti and Maioli, 1989). By preemptively recruiting appropriate muscle forces to not only position the hand but to also stiffen the limb and compensate for impact, the control system can minimize the limb displacement upon impact. In this framework, sensory feedback provides a means of error correction and performance assessment while the feedforward mechanisms execute a motor plan. These predictive processes have been associated with the primary motor cortex (Gritsenko et al., 2011a), brainstem (Prentice and Drew, 2001), supplementary motor cortex (Richard et al., 2017), spinal cord (Prentice and Drew, 2001), and the cerebellum (Shadmehr et al., 2010; Richard et al., 2017).

\section{Internal Models}

The mechanisms of feedforward and feedback control create an integrated control system that can both anticipate expected limb dynamics and respond to unexpected disturbances. One method for achieving this system is by a neural representation of an internal model of limb dynamics (Wolpert and Kawato, 1998; Wolpert et al., 1998; Kawato, 1999). The internal model could predict the expected motor output given a particular input (i.e. motor intent) as well as the expected sensory feedback. During movement execution, this expected sensory feedback is compared to actual feedback. Discrepancies between the expected vs actual feedback are then used to 1) adjust and/or update the motor plan and 2) update the internal model. This process provides a means for both error correction and the acquisition of new movements or novel dynamics. 
Neuromechanical Tuning Hypothesis

The neural strategy that leads to the selection and recruitment of muscles must consider the dynamic characteristics of motoneuron recruitment, muscle force generation, muscle moment arms, and both intrinsic and extrinsic sources of joint torques. The neuromechanical tuning hypothesis states that the mechanical characteristics of movement are embedded within the neural hierarchy (Prochazka and Yakovenko, 2007a). This hypothesis suggests that these properties must be incorporated into the motor system's internal model, which can then be refined or adapted via sensory feedback.

\section{Dissertation Summary}

In this dissertation, I explored the role of limb dynamics and musculoskeletal properties in the formation of motor commands. Chapter 2 describes the development and validation of a musculoskeletal model of the shoulder and the implications that these dynamics may have for muscle recruitment. Chapter 3 describes a computational and experimental study in which I used a mathematical approximation of muscle spindle primary afferent activity to determine whether the monosynaptic feedback pathway could shape motoneuronal recruitment during voluntary movements. Finally, Chapter 4 describes a study of the descending corticospinal contribution to the control of dynamically distinct reaching tasks.

\section{Chapter 2 - Biomechanical constraints underlying motor primitives derived from the musculoskeletal anatomy of the human arm}

(this chapter has been adapted from Gritsenko V, Hardesty RL, Boots MT, Yakovenko S (2016) Biomechanical constraints underlying motor primitives derived from the musculoskeletal anatomy of the human arm. PLOS ONE 11:1-18. The subsection "Additional Model Development" contains both model development and analysis beyond the publication.)

\section{Abstract}

Neural control of movement can only be realized though the interaction between the mechanical properties of the limb and the environment. Thus, a fundamental question is whether anatomy 
has evolved to simplify neural control by shaping these interactions in a beneficial way. This inductive data-driven study analyzed the patterns of muscle actions across multiple joints using the musculoskeletal model of the human upper limb. This model was used to calculate muscle lengths across the full range of motion of the arm and examined the correlations between these values between all pairs of muscles. Musculoskeletal coupling was quantified using hierarchical clustering analysis. Muscle lengths between multiple pairs of muscles across multiple postures were highly correlated. These correlations broadly formed two proximal and distal groups, where proximal muscles of the arm were correlated with each other and distal muscles of the arm and hand were correlated with each other, but not between groups. Using hierarchical clustering, between 11 and 14 reliable muscle groups were identified. This shows that musculoskeletal anatomy does indeed shape the mechanical interactions by grouping muscles into functional clusters that generally match the functional repertoire of the human arm. Together, these results support the idea that the structure of the musculoskeletal system is tuned to solve movement complexity problem by reducing the dimensionality of available solutions.

\section{Introduction}

Movements are the product of interactions between neural control signals and the musculoskeletal dynamics that depend on limb anatomy (Yakovenko, 2011). This complex dynamical system depends on the active and passive forces that arise directly or indirectly from muscle contractions and segmental inertia, and requires complex control by the neural motor system. The skeletal limb structure can simplify the control complexity, for example locomotor dynamics is stabilized by advantageous passive dynamics (Collins, 2005). Musculoskeletal morphology has traditionally been viewed as an additional complexity with redundant characteristics that the central nervous system (CNS) is required to solve (Bernstein, 1967). However, evidence has been mounting for the simplifying role of muscle anatomy through increased stability due to viscoelastic properties, which help resist perturbations (Asatryan and 
Feldman, 1965; Brown and Loeb, 2000; Yakovenko et al., 2004; Prochazka and Yakovenko, 2007a; Valero-Cuevas et al., 2015). These properties may even contribute to shaping the multidimensional and state-dependent control parameter space for volitional movements in the "uncontrolled manifold" theory (Latash et al., 2002). In particular, Kutch and Valero-Cuevas have suggested that muscular anatomy may help reduce the dimensionality of control space through mechanical coupling even in the absence of a common neural command (Kutch and ValeroCuevas, 2012). However, the extent and topography of muscle coupling across more than several muscles has not been previously described. In the current study, we have used an inductive datadriven approach to further test this idea and to quantify the dimensionality reduction accomplished by the mechanical coupling of muscle actions across the physiological range of arm and hand postures using a validated dynamic musculoskeletal model (Thelen and Anderson, 2006; Delp et al., 2007; Saul et al., 2015b).

Muscles have been traditionally classified into agonist and antagonist pairs using their anatomy (Lombard, 1903; Kuo, 2002) or innervation and participation in sensory-evoked actions (Sherrington, 1909, 1910). For example, stimulation of sensory pathways activates ilia-psoas, tibialis anterior, and extensor digitorum longus that together participate in flexion of hip and ankle of the lower limb (Yakovenko et al., 2004). Using this definition, excitation and inhibition patterns give the physiological binary membership of muscles in mutually-opposing functional groups. This idea has been extended further to the concept of motor primitives or synergies, where a smaller subset of grouped muscle actions can accomplish a variety of tasks (Patla, 1985; Bizzi et al., 1991; Giszter et al., 1993). Alternatively, the anatomical joint-based nomenclature can be used to identify muscle actions around specific joints. For example, the biceps brachii and triceps brachii act as antagonists around the elbow, because the former causes elbow flexion, while the latter causes elbow extension. The latter definition does not rely on neural activations and is purely due to the anatomy of muscle origins and insertions on the bone and their moment arms around the 
joints. In this study, our goal was to quantify mechanical coupling that underlies the basic functionality and dimensionality of the musculoskeletal system and represents the lowest hierarchical level of movement control. This coupling constrains neural actions and, thus, bears directly on the concept of motor primitives or synergies.

\section{Methods}

\section{Model}

The musculoskeletal model based on the dynamic upper limb model created by Saul et al. (Saul et al., 2015b) was constructed in OpenSim (version 3.0, Stanford University, Stanford, CA, USA) (Fig. 2-1) and modified in several aspects. Separate bodies for each segment of the hand digits were created to recreate an additional 16 DOFs of the human hand. Metacarpals of digits 2 through 5 (index through little fingers) were modeled as a single body with the inertia of a right rectangular prism. All carpometacarpal joints but the first one were represented by a single wrist joint with 2 DOFs. These corresponded to the rotations between the fused metacarpals $2-5$ and ulna coordinate systems around the x-axis for flexion/extension (Fig. 2-1C). Pronation and supination was achieved by the rotation of radius around ulna as in the published model. The first carpometacarpal joint of the thumb was modeled with 2 DOFs. These corresponded to the rotations between the first proximal phalanx and radius coordinate systems around the $\mathrm{x}$-axis for flexion/extension and around the $Z$ axis for abduction/adduction. A single DOF (flexion/extension) was assigned to all metacarpophalangeal joints corresponding to the rotations around the x-axes of the coordinate systems of the proximal phalanges 2-5 and the corresponding metacarpals (Fig. 2-1C). Phalanges were modeled as cylinders with lengths and radii of a human subject. A single DOF (flexion/extension) was assigned to all proximal and distal interphalangeal joints. The axes of rotations of all joints of the arm, with the exception of pronation/supination of the forearm, were adjusted to correspond to Euler angles between adjacent body Cartesian coordinate systems (Fig. 2-1C) to maximize the utility of this analysis for forward and inverse dynamics, where the 
motion is described in terms of changes in joint Euler angles caused by muscle and inertial torques. The total number of model DOFs, including the arm and hand, was 23. The list of abbreviated names of musculotendinous actuators included in the model and the muscles they represent is in Table 1. Two intrinsic hand muscles, the Opponens Pollicis (OP) and Flexor Pollicis Brevis (FPB), were added to the published model, with their origin and insertion points estimated from Gray's anatomy [23].



Figure 2-1: Illustration of the model and local coordinate systems.

(A) and (B) Musculotendinous paths from anatomical origins to insertions on the skeleton are illustrated with red lines with selected labels. (C) Coordinate systems for each segment are illustrated with the colorcoded cartesian exes in red, yellow, and green for $x-, y$ - and z-axes respectively. Euler angles around these axes represent joint angles. The illustrated posture of the model corresponds to all joint angels at zero. The local coordinate systems are shown only for thumb and index finger. The coordinate systems of the other digits follow the orientation of the coordinate systems for the index finger.

\section{Human subjects}

This research was approved by the West Virginia University Institutional Review Boards (IRBs) for Protection of Human Research Subjects (protocol number 1311129283A004). Informed written consent was obtained on the forms approved by IRBs from 10 healthy young human subjects. The subjects were 5 males and 5 females of mean age $26.2 \pm 6.2$ (standard deviation, SD) years, mean weight $77.5 \pm 14.1 \mathrm{~kg}$, and mean height $1.74 \pm 0.04 \mathrm{~m}$. In addition to participant height and weight, the lengths of all major arm segments represented as individual bodies in our 
model were measured (Table 1). These measurements were used to scale the model (subject 0 ) to the dimensions of each individual (subjects 1-10). Each of the model segments and origins and insertions of all muscles were scaled proportionally to the length of each subject's segment (Delp et al., 2007).

\section{Analysis of mechanical coupling}

We calculated musculotendinous lengths (referred to as muscle lengths) across the full range of motion of the arm using MATLAB (MathWorks Inc.) pipeline tools of OpenSim by permuting postures through all joint excursion combinations within the physiological range of motion in $20 \%$ increments. The obtained muscle length data about each of the DOFs at each posture for each muscle of each individually scaled model were then passed through a regression analysis to explore the relationships between muscle lengths for each subject. In this analysis, the correlation coefficients $(r)$ for muscle lengths between all pairs of muscles across all postures were calculated. Due to computational limitations associated with the multidimensional datasets, a random selection of up to 10,000 postures to describe all possible arm and hand state variations was used for the mechanical coupling analysis (see below). Postures when both shoulder abduction and flexion angles were above 90 degrees were excluded from the analysis due to limitations of a gimbal joint. All correlations between muscle lengths were done using 1,000 postures randomly selected from the full dataset. This number of postures was selected because the residual unexplained variance $\left(1-r^{2}\right)$ at this and higher numbers of postures approached zero (Fig. 2-2). 


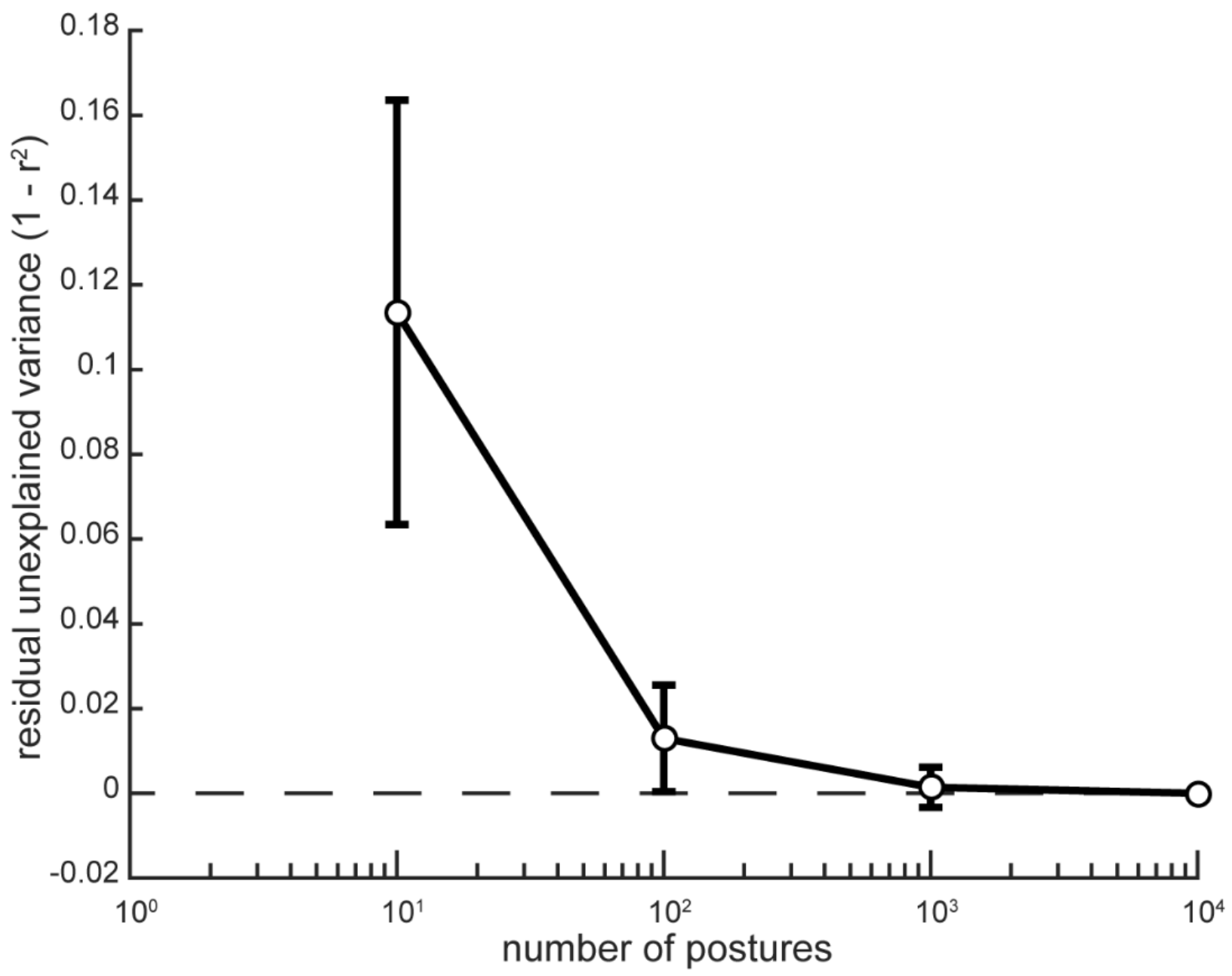

Figure 2-2: The difference between $r 2$ values for the correlations between muscle lengths as a function of the number of selected postures

Error bars show standard deviations around the mean.

The agonistic and antagonistic relationships between the muscles of each subject were quantified using hierarchical clustering of the muscle length correlation matrix in MATLAB. Hierarchal clustering was applied to all muscles and separately to only distal muscles. The criterion for inclusion into distal (hand-related) or proximal (shoulder-related) clusters was the level of muscle length correlation between the muscle of interest and either the muscles spanning the shoulder joint or the muscles spanning the wrist joint in all subjects. For clustering, the correlation matrix was transformed into the heterogeneous variance explained (HVE) as described next. The transformation ensured that agonist muscle pairs grouped together, i.e. had small distance values 
in proportion to shared variance, whereas antagonist muscles appeared relatively far apart, i.e. had larger distance values. Agonist muscles were characterized by positive r-values, and antagonists were characterized by negative $r$-values. The coefficient of determination $\left(r^{2}\right)$ was used in the HVE equation as the measure of shared variance between the changes in lengths of muscle pairs. The HVE for agonists was thus set to be equal to $\left(1-r^{2}\right)$, while the HVE for antagonists was equal to $\left(1+r^{2}\right)$. This resulted in agonist muscle pairs with large positive r-values being defined by short distances close to 0 , while antagonist muscle pairs with large negative $r$ values were defined by long distances close to 2 . Zero or insignificant correlations were defined by intermediate distances close to 1 . Hierarchical clustering was applied using the linkage function with unweighted average distance method to the HVE matrix to identify between 2 and 20 clusters in each subject. The reliability of clustering was evaluated based on the number of muscles that did not fall into the same cluster across subjects. Trivial results with single-muscle clusters were excluded from the reliability analysis.

Unless otherwise stated, all data is referenced by mean \pm SD.

\section{Results}

The musculoskeletal model comprised 52 musculotendinous actuators (model muscles) that spanned 23 DOFs. Of the 52 actuators, 26 represented compartments of 7 muscles, e.g. 3 triceps actuators representing long, lateral, and medial heads of the triceps brachii. Thus, the model represented the anatomical arrangement of 33 individual muscles. There were 15 actuators that spanned only the shoulder joint (3 DOFs), 3 actuators that spanned both the shoulder and elbow (4 DOFs) joints, 6 actuators that spanned only the elbow joint (2 DOFs due to flexion-extension and pronation-supination), and 8 actuators that spanned both the elbow and wrist (3 DOFs, not including pronation/supination) joints, with the remaining 20 actuators spanning the wrist and at least 1 finger joint. Thus, most muscles were associated with several DOFs. For example, the length of the pronator teres depends on the angles of forearm pronation/supination and elbow 
flexion/extension shown in Figure 3. The lengths of the actuators changed non-linearly as a function of the DOFs they controlled, as do their moment arms (Sartori et al., 2012). This implies that a constant activation of a given muscle results in a different contribution of that muscle to the net joint torque when the arm is held at different postures or throughout the motion. These nonlinearities are the result of complex anatomical paths that the muscles take as they wrap around each joint, particularly joints with multiple DOFs. 
Musculotendinous length of pronator teres muscle
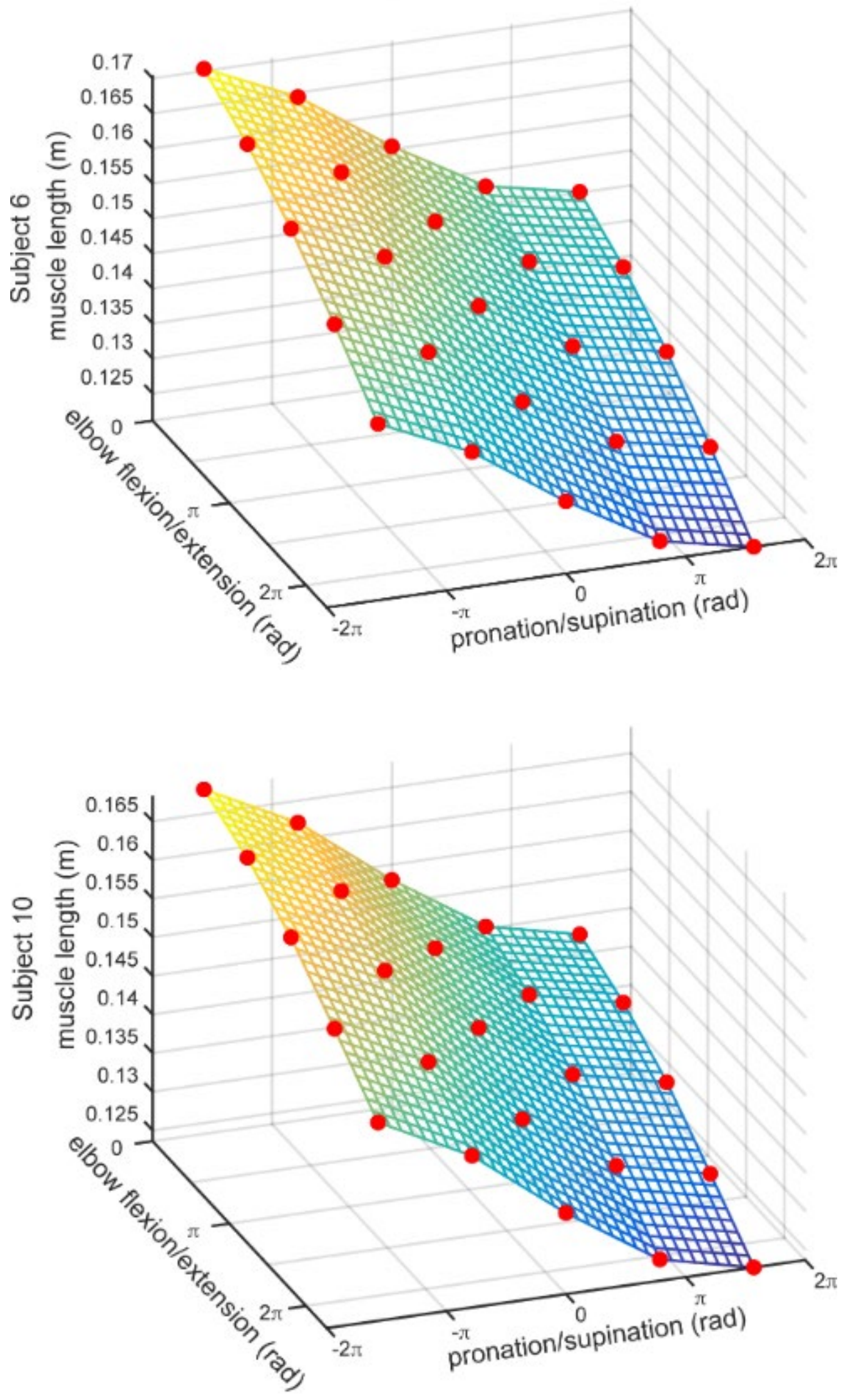
Figure 2-3: Examples of muscle lengths for the pronator teres, a single 2-DOF muscle originating on the humerus and attaching on the radius, in two subjects

The data points (circles) correspond to muscle lengths throughout the physiological range of motion for each DOF.

The action of each musculotendinous actuator in the model depends on its attachment to the bones and the path it takes around the joint. These data are based on human anatomical data (Saul et al., 2015b). To investigate the effect of individual skeletal proportions on mechanical coupling, the lengths of arm segment were scaled to the values from each of 10 human subjects. This changed the values for muscle lengths associated with each arm posture. The skeletal proportions across subjects varied with SD, ranging from $5 \%$ to $27 \%$ of the average segment length (Table 2). However, the relationships between muscle lengths were highly stable across subjects, as described in detail in the following sections.

As expected, the muscle lengths across muscles were highly correlated in agonistic or antagonistic fashion (Fig. 2-4A). Positive correlations indicate that the muscle length increases or decreases together, representing agonistic action across multiple arm postures (Fig. 2-4B). Here, the method is limited to the examination under the isometric condition that does not take into account dynamics or history-dependent muscle properties (Joyce and Rack, 1969; Rack and Westbury, 1974; Gillard et al., 2000). Negative correlations indicate coincident increase of one muscle length while the other is decreased, representing antagonistic action. Not surprisingly, the lengths of all actuators representing compartments of the same muscle were highly correlated (bright yellow squares around the unity line in Fig. 2-4A). Surprisingly, however, most of the muscles showed strong correlations that broadly formed two large clusters, where proximal muscles of the arm were correlated with each other and distal muscles of the arm and hand were correlated with each other, but not as much with the proximal cluster. For example, the length of LATD_M was highly correlated with that of PECM_C $\left(r^{2}=0.594\right)$, but the correlation with the distal cluster was minimal $\left(r^{2}=0.004\right.$ with FDP5). Similarly, the length of ED5 was highly correlated with that of ED_M $\left(r^{2}=0.793\right)$, but the correlation with the proximal cluster was minimal $\left(r^{2}=0.004\right.$ with 
LATD_C). This is the first time the agonistic and antagonistic actions of muscles have been quantified across the whole workspace of the human arm. 
A Correlation matrix for musculotendinous lengths of all muscles

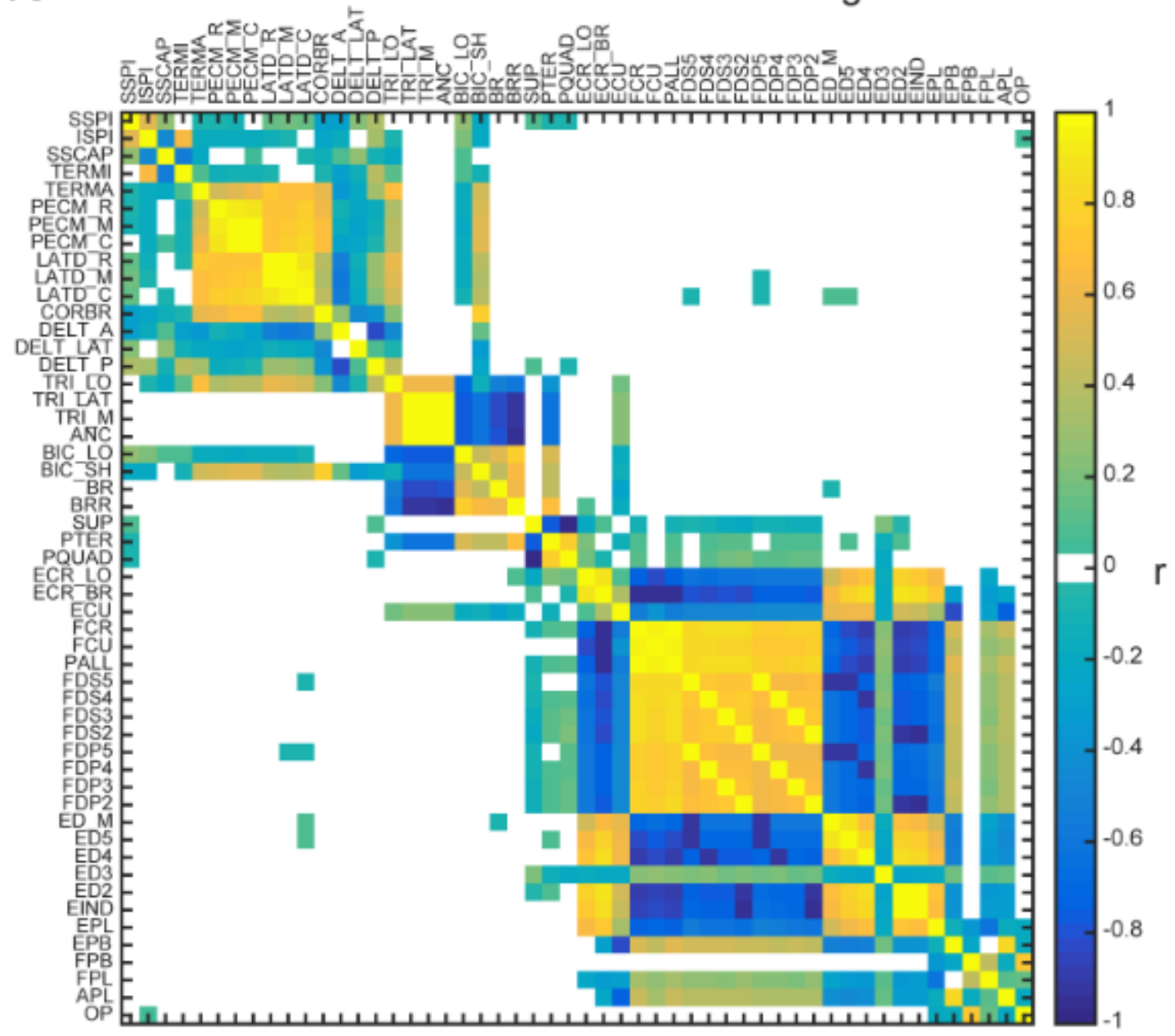

B Histogram of signicant Pearson correlation coefficents




Figure 2-4: Examples of the correlations between muscle lengths in a single subject

Only significant correlations are plotted $(p<0.05)$. (A) Pearson correlation coefficient $(r)$ between muscle lengths of all muscle pairs. Blue colors indicate negative correlations; yellow colors indicate positive correlations. (B) Histogram of $r$-values for each subject across all muscle pairs. The bar plots are binned with 0.2 increments, and only significant values were included in the analysis.

The hierarchical clustering analysis of muscle lengths quantitatively identified muscle groups at multiple levels of detail. The first 2 clusters in all subjects represented broadly flexor and extensor actions across all joints or DOFs (Fig. 2-5C, dark blue and red clusters emanating from the center). However, two groups were insufficient for the consistent classification of all muscles across subjects. Some muscles may be classified differently for different subsets of subjects. For example, the subgroup that contains latissimus dorsi and pectoralis major was clustered either with extensors in 5 out of 11 subjects or with flexors in the rest of the subjects (see Fig. 2-5C, subgroup marked * in two different subjects). Note that the composition of this subgroup remained unchanged. The separate analysis of distal musculature showed the same pattern of clusters as the analysis of all muscles. For example, the same subgroup consisting of thumb muscles remained unchanged in both analyses (see Fig. 2-5C, subgroup marked ${ }^{\wedge}$ in the same subject). 

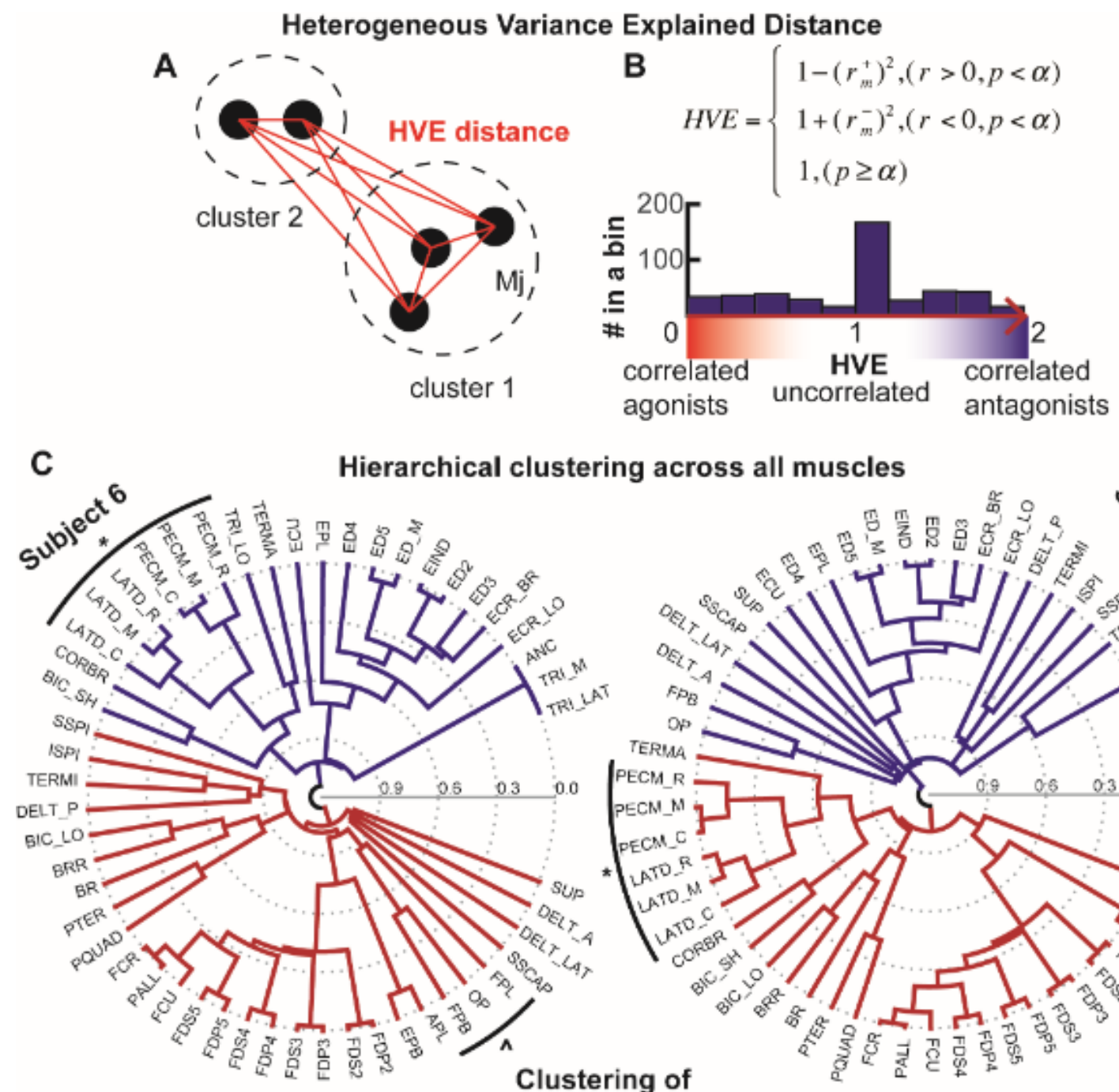

ing across all muscles


only distal muscles

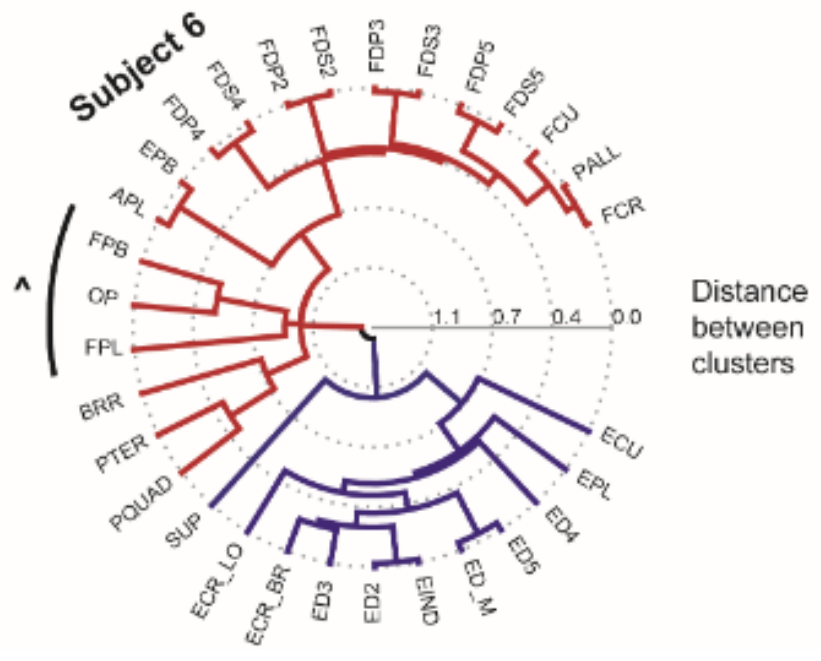

Figure 2-5: Hierarchical clustering methodology and examples for two subjects 
(A) Geometric illustration of heterogenous variance explained (HVE). HVE distance is determined by the correlations of musculotendon length between muscle pairs determined by the equation in (B). (B) The equation for calculating HVE distance. The negative regressions $\left(r^{-}\right)$indicate opposite or antagonistic actions of muscle pairs, when the positive ones $\left(\mathrm{r}^{+}\right)$correspond to the synergistic or agonistic actions. Insert shows a histogram of HVE values for one subject across all muscle pairs. (C) Examples of hierarchical clustering for individual subjects. Clustering across all muscles is shown in the top two polar dendrograms. The bottom plot shows clustering across only the distal muscles for one of the subjects. Lines emanating from the center indicate the distance between muscle clusters calculated from HVE. The main agonistantagonist division can be established using a high clustering threshold (2 clusters with dark red and dark blue lines), and further subdivisions are revealed by the progressive lowering of the threshold. Example matching clusters are marked by outside brackets with * ${ }^{*}{ }^{\wedge}$.

The consistency of muscle cluster assignment across subjects changes as a function of the number of clusters selected in the analysis (Fig. 2-6). The number of unclassified muscles was generally high when muscles were divided into 3 to 8 clusters, which means less consistent clusters across subjects (Fig. 2-6A). This followed by a plateau of 9 to 13 more consistent clusters, in which the same muscle groups were identified across subjects. Further subdivision into more than 13 clusters generated increasingly more trivial results with single-muscle clusters, which is evidenced by increasing normalized number of unclassified muscles (Fig. 2-6A, right plot). When the inclusion threshold for cluster assignment across subjects was increased from $50 \%$ (muscle belongs to the same cluster in $50 \%$ of subjects) to $100 \%$ (muscle belongs to the same cluster in all subjects), the number of unclassified muscles changed for the different numbers of clusters. All muscles were classified into the same clusters in at least half of all subjects when 2 or $9-16$ clusters were selected (Fig. 2-6A, dark blue line). The increase in the inclusion threshold to $100 \%$, i.e. the muscle had to belong to the same cluster across all subjects, increased the peak number of unclassified muscles from 15 to 30 (Fig. 2-6A red line on left plot). The most reliable number of clusters, based on the minimal number of unclassified muscles across all thresholds, was 11 (Fig. 2-6A, black arrows). Normalizing the number of unclassified muscles to cluster size did not change this estimate (Fig. 2-6A, right). Similar trends were seen in the reliability of clustering of distal muscles (Fig. 2-6B). Here, the most reliable number of clusters was 6 (Fig. 2-6B, black arrows). This analysis identified the minimum number of reliable clusters, which are illustrated on the mean polar dendrogram across all subjects in Figure 2-7. These clusters of muscles that span 
multiple joints represent the simplest actions that can be accomplished through mechanical coupling.

\section{A Clustering across all muscles}
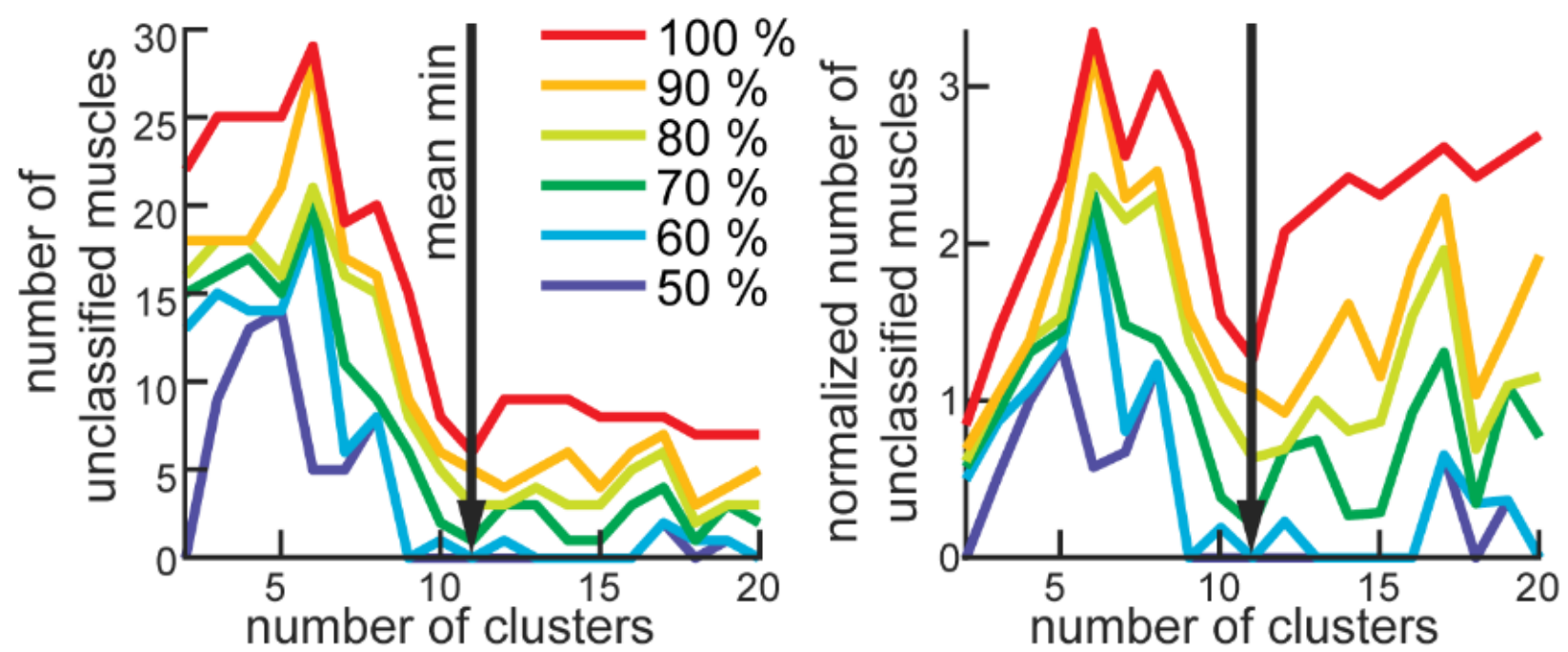

B Clustering across only distal muscles
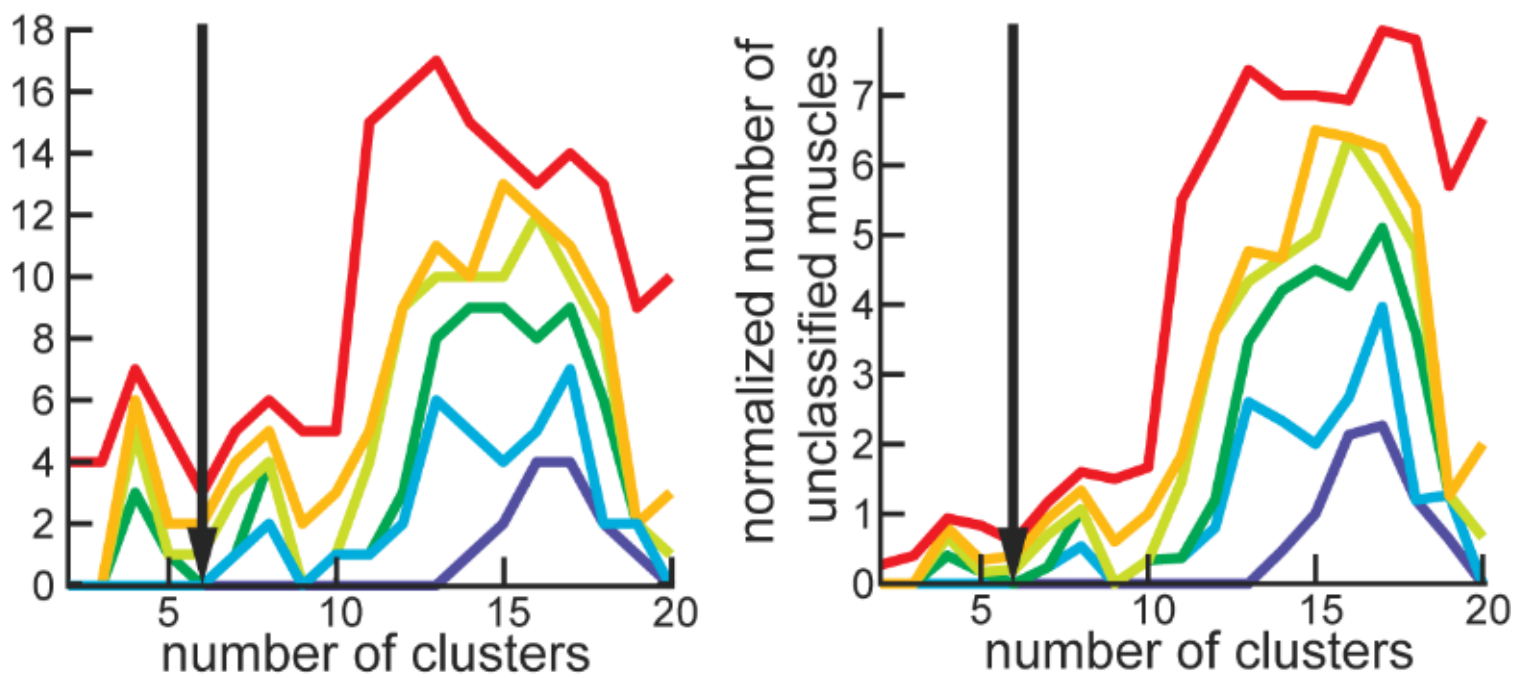

Figure 2-6: Reliability of clustering across subjects

(A) The average number of unclassified muscles is shown as a function of the number of clusters. Each colored line corresponds to the level of stringency for the variability in classification across subjects, e.g. $100 \%$ stringency corresponds to the same classification in all subjects. The right panel shows the same values normalized to the average number of muscles in all clusters. (B) The same analysis as in A for distal muscles only. Vertical black arrow indicates the nontrivial minimum for the number of clusters (11 clusters for all and 6 clusters for distal muscles), which represents the most reliable number of muscle clusters. 


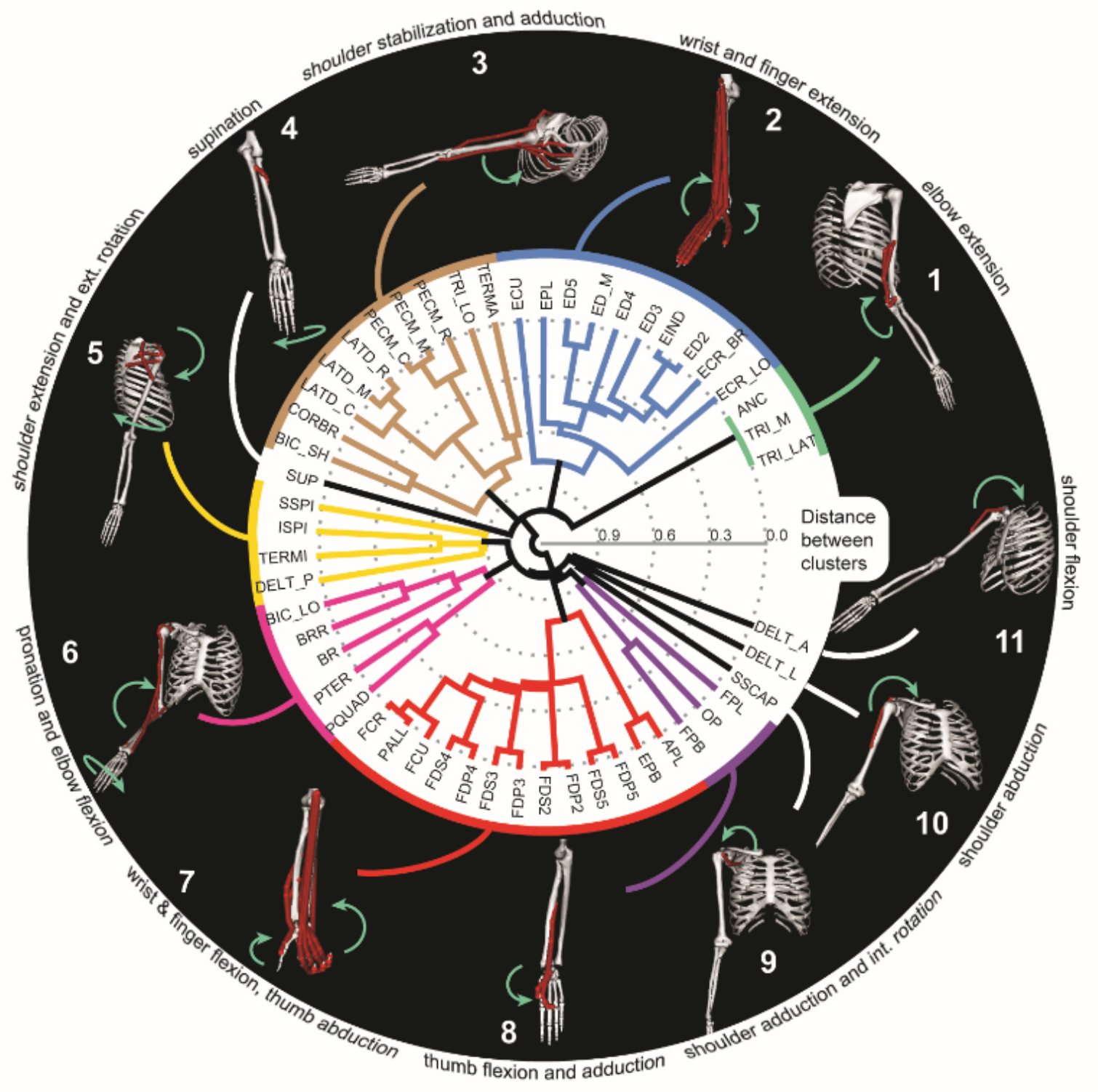

Figure 2-7: Mean hierarchical clustering across all subjects

The polar dendrogram illustrates hierarchical clustering as described in Fig. 2-5C. Inserts along the perimeter illustrate the directions of motion (green arrows) produced by the activation of muscles in the model shown in Fig. 2-1. Only muscles that belong to the corresponding cluster are shown on each insert.

\section{Discussion}

In this work, we have described for the first time the low-dimensional structure of agonistic or antagonistic mechanical actions, termed the mechanical coupling, of major arm and hand muscles 
across their physiological range of motion. We demonstrated that a low-dimensional structure emerges even from the musculoskeletal anatomy without the presence of common neural feedforward or feedback signals (Fig. 2-7). We found that there exists an optimal range for the number of clusters that reliably group muscles according to actions (Fig. 2-6). Thus, these results may help us address the unresolved controversies associated with the definition of motor primitives by detailing the lowest level in the bottom-up organization of the motor control system. This mechanical coupling between muscles defines the natural repertoire of actions that the musculoskeletal system can produce in presence of inertial and gravitational forces, external perturbations, and neural control signals. Therefore, our results provide further evidence to support the idea that musculoskeletal anatomy helps to reduce the dimensionality of control space through the mechanical coupling (Asatryan and Feldman, 1965; Brown and Loeb, 2000; Yakovenko et al., 2004; Prochazka and Yakovenko, 2007a; Kutch and Valero-Cuevas, 2012; Valero-Cuevas et al., 2015)

One prevalent theoretical explanation of how the nervous system resolves limb control problems is based on the idea of motor primitives, i.e. groups of muscles sharing the same common source of neural activation (Bizzi et al., 1991; Giszter et al., 1993; Tresch et al., 1999; d'Avella et al., 2003a). Inherent in this concept is the idea that motor primitives reduce the complexity of neural control signals by enabling the production of any movement from a smaller selection of control actions (Bizzi et al., 1991; Giszter et al., 1993). However, the theory of motor primitives, or synergies, defined this way has recently come under increased scrutiny due to the indivisible interaction and mutual dependency between neural control of muscle activations and biomechanics of the resulting movement (Tresch and Jarc, 2009). These interactions and dependencies may emerge in the synergy analyses when limb movement engages sensory feedback from mechanically coupled muscle groups (Valero-Cuevas et al., 2015) or, alternatively, constitute evidence for common feedforward drive within neural code (Overduin et al., 2015; Rana 
et al., 2015; Yakovenko and Drew, 2015). The common neural drive would also originate if the neural networks are embedding movement dynamics for processing motor commands. The concept of central pattern generators (CPG) in the spinal cord, in particular, is a representative example of low-dimensional neural processing for rhythm generation that is coupled to mechanical oscillations between limbs and the environment to produce locomotion (Prochazka and Yakovenko, 2007a). Also, the evolving predominant view is that neural processing can be represented by a dynamical system acting through available neuromuscular elements to generate appropriate signals for desired movements (Shenoy et al., 2013). Taken together, neural activity within the hierarchical CNS contains the representation of downstream processing that may reflect the low-dimensional representations of targeted mechanisms resulting in neural signals consistent with the idea of common drive.

The neuromechanical tuning may be used to redefine motor primitives in terms of individual actions being controlled. The hierarchal structure of both the neural motor system and the mechanical coupling implies that the control complexity can be broken down into specific actions produced by common signals to muscle groups at different levels of the identified mechanical coupling hierarchy. Then, CPGs in the spinal cord, which are modeled as a dynamical system (Yakovenko, 2011), could be viewed as neural motor primitives that are entrained with the inverted pendulum oscillator formed by the mechanical interactions of limbs with the ground (Taga et al., 1991a; Full and Koditschek, 1999; Prochazka and Yakovenko, 2007a). Because the entraining originates in the sensors associated with muscles, the musculoskeletal organization has bearing on this unit of control. The CPG generates antagonistic activity that results in gross mechanical oscillatory actions through interactions between antagonistic groups of muscles (Yakovenko et al., 2002). The CPGs are also thought to contribute to arm motor control (Taga et al., 1991a; Full and Koditschek, 1999; Prochazka and Yakovenko, 2007a; Zehr et al., 2007; Drew and Marigold, 2015). The antagonistic groups observed in our analysis as the first two clusters in 
the mechanical coupling diagram may reflect the same concept (Fig. 2-5C). When dexterous movements are required, e.g. to step over obstacles during locomotion or reaching, the gross CPG motor primitive must be fractioned into smaller components specific to the task (Yakovenko and Drew, 2015). In our analysis, this would be equivalent to following the polar dendrogram from the center with gross representations to periphery with fractured fine representations (Fig. 2-7). The neighboring fine motor primitives in our analysis could be combined to represent functional movements. Defensive limb movements can be generated by three combinations of 10,5 , and 6 groups; feeding movement can arise from the recruitment of all groups in 6 - 8; and the manipulation movements can be generated by four combinations 2,6 , and 11 , followed by 7 for grasping. While these combinations are qualitatively similar to those observed in response to the long-train intracortical microstimulation of the motor cortex (Graziano et al., 2002; Graziano and Aflalo, 2007; Stepniewska et al., 2009), the link between neural activity and the composition of coupled muscle groups remains to be tested in future studies.

Another result in this study is the salient separation between muscle motor primitives of proximal and distal arm joints. This is unexpected, because the subsets of proximal and distal muscles span the same elbow joint and contribute to pronation/supination DOF. Only sparse correlations between the pairs of muscles spanning primarily proximal and primarily distal joints are present in our study (Fig. 2-4). This result indicates that the anatomical arrangement of muscles is consistent with the idea of two distinct control targets: proximal arm and distal hand groups. Coincidentally, the spatiotemporal separation between the activation of proximal and distal muscles is present in goal-directed reaching movements that are traditionally separated into two phases: gross arm motion to transport the hand to the desired location and fine hand motion to manipulate objects. It has also been suggested that these phases are controlled separately by the nervous system (Jeannerod et al., 1998; Kawato and Samejima, 2007). Such muscle organization and the possible separation within neural control pathways may be the result of 
evolutionarily-driven expansion of distal musculature to enable the increased dexterity of object manipulation characteristic of primates. The spatiotemporal separation of muscle activity during limb transfer, generally controlled by proximal muscles, and limb placement, generated by distal musculature, is also evident in the regulation of evolutionarily connected phases of reaching movement and precise modifications in quadruped stepping. Moreover, these separate temporal phases are correlated to the activity of distinct corticospinal circuits (Yakovenko and Drew, 2015).

Our analysis uses the incidence of length excursions in different postures as a measure of functional similarity in muscle actions. The analysis is based on sampling representative postures within the physiological range of motion (ROM); yet, this posture space may not be functionally homogenous. It included both likely and unlikely joint configurations based on the frequency of observing their representation in daily use (Ingram et al., 2008; Howard et al., 2009). For these subsets of joint configurations there may exist distinct relationships within subsets of muscles. The method of uniform sampling used here may not capture the coupling or uncoupling among the muscle pairs within these subsets of likely and unlikely postures. Then there may also be a subset of muscles with changing relationships within different postures. Because these muscle pairs would have low correlations in our analysis, the only groups that could be affected would be those associated with the weak relationships between antagonistic muscles acting on scapular (groups $3 \& 5$ of Fig.2-7). Fig. 2-4 shows that these are the only large groups with r-values within medium to low correlations, i.e. between -0.5 and 0.5 values, that may be affected. It is tempting to speculate that the proximal arm muscles may change their functional affiliation based on the familiarity with task. This could be reflected in different biomechanical advantages or affordances that influence movement planning (Cos et al., 2014). This question will be addressed in the future research. In the presented analysis, the correlations across postures indicate the shared dependence on joint constraints to define functionally similar muscles over the full physiological ROM that includes all possible limb postures with the exclusion of extremes. 
Several methods are commonly used to derive motor primitives from muscle activity, and all rely on extracting shared signal redundancy among neural discharge and/or muscle activity (d'Avella et al., 2003a; Tresch et al., 2006; Cheung et al., 2009; Yakovenko et al., 2011; Krouchev and Drew, 2013; Yakovenko and Drew, 2015). Cumulatively, these studies support the idea that muscle motor primitives are reflected in the neural activity; however, the confounding factors may offer alternative explanations for coupled activity (Kutch et al., 2008; Kutch and Valero-Cuevas, 2012; Valero-Cuevas et al., 2015). The mechanical coupling derived from the correlations of muscle lengths across physiological postures qualitatively matches the groups observed in the decomposition analyses. For example, the biceps long, brachioradialis, brachialis, and pronator teres are in the same muscle group 6 (Fig. 2-7) and are also part of the W1 synergy identified with time-varying synergy analysis (d'Avella et al., 2006). Similarly, the teres major and latissimus dorsi are part of a single muscle group 3 and posterior deltoid is a part of an adjacent group 5 identified through the mechanical coupling analysis (Fig. 2-7) and are also part of the W5 synergy identified with time-varying synergy analysis (d'Avella et al., 2006). This result is consistent with observations that the underlying musculoskeletal dynamics can constrain the space of neural commands to a low-dimensional subspace identified with decomposition methods (Kutch and Valero-Cuevas, 2012). Thus, the existence of the mechanical coupling of muscles generally agrees with the findings of alternative methods.

In conclusion, our analysis of arm and hand muscles is a quantitative description of the functional organization within the musculoskeletal system that contributes to the concept of motor primitives. The organization of movement derived from the musculoskeletal architecture offers a novel perspective on the motor control problem solved by CNS. 


\section{Tables}

Table 1: The abbreviations of muscles included in the analyses.

\begin{tabular}{|c|c|c|c|}
\hline $\begin{array}{l}\text { Muscle } \\
\text { Abbreviation }\end{array}$ & Muscle Name & $\begin{array}{l}\text { Muscle } \\
\text { Abbrevia } \\
\text { tion }\end{array}$ & Muscle Name \\
\hline DELT_A & Deltoid (anterior) & FCR & Flexor Carpi Radialis \\
\hline DELT_L(AT) & Deltoid (lateral) & FCU & Flexor Carpi Ulnaris \\
\hline DELT_P & Deltoid (posterior) & PALL & Palmaris Longus \\
\hline SSPI & Supraspinatus & PTER & Pronator Teres \\
\hline ISPI & Infraspinatus & PQUAD & Pronator Quadratus \\
\hline SSCAP & Subscapularis & FDS5 & Flexor Digitorum Superficialis (5th digit) \\
\hline TERMI & Teres Minor & FDS4 & Flexor Digitorum Superficialis (4th digit) \\
\hline TERMA & Terer Major & FDS3 & Flexor Digitorum Superficialis (3rd digit) \\
\hline PECM_R & Pectoralis Major (rostral) & FDS2 & Flexor Digitorum Superficialis (2nd digit) \\
\hline PECM_M & Pectoralis Major (medial) & FDP5 & Flexor Digitorum Profundus (5th digit) \\
\hline PECM_C & Pectoralis Major (caudal) & FDP4 & Flexor Digitorum Profundus (4th digit) \\
\hline LATD_R & Latissimus Dorsi (rostral) & FDP3 & Flexor Digitorum Profundus (3rd digit) \\
\hline LATD_M & Latissimus Dorsi (medial) & FDP2 & Flexor Digitorum Profundus (2nd digit) \\
\hline LATD_C & Latissimus Dorsi (caudal) & ED5 & Extensor Digitorum (5th digit) \\
\hline CORBR & Coracobrachialis & ED4 & Extensor Digitorum (4th digit) \\
\hline TRI_LO & Triceps (long) & ED3 & Extensor Digitorum (3rd digit) \\
\hline TRI_LAT & Triceps (lateral) & ED2 & Extensor Digitorum (2nd digit) \\
\hline TRI_M & Triceps (medial) & ED_M & Extensor Digitorum Minimi \\
\hline ANC & Anconeus & EIND & Extensor Indicis \\
\hline SUP & Supinator & EPL & Extensor Pollicis Longus \\
\hline BIC_LO & Biceps Brachii (long) & EPB & Extensor Pollicis Brevis \\
\hline BIC_SH & Biceps Brachii (short) & FPL & Flexor Pollicis Longus \\
\hline $\mathrm{BR}$ & Brachialis & APL & Abductor Pollicis Longus \\
\hline BRR & Brachioradialis & OP & Opponens Pollicis \\
\hline ECR_LO & Extensor Carpi Radialis Longus & FPB & Flexor Pollicis Brevis \\
\hline ECR_BR & Extensor Carpi Radialis Brevis & ECU & Extensor Carpi Ulnaris \\
\hline
\end{tabular}


Table 2: The summary of anthropometric measurements. All distance measurements, unless indicated otherwise in brackets, were made between the estimated centers of joint rotation.

\begin{tabular}{|c|c|c|}
\hline Segment name & Length (m) & Length ( $\%$ of subject height) \\
\hline Thorax & $0.217 \pm 0.032$ & $12.5 \pm 1.9$ \\
\hline $\begin{array}{l}\text { Shoulder (between clavicle } \\
\text { and scapula acromial tip) }\end{array}$ & $0.194 \pm 0.016$ & $11.2 \pm 0.8$ \\
\hline Humerus & $0.279 \pm 0.026$ & $16.1 \pm 1.4$ \\
\hline Ulna & $0.262 \pm 0.014$ & $15.1 \pm 0.8$ \\
\hline Radius & $0.262 \pm 0.014$ & $15.1 \pm 0.8$ \\
\hline $\begin{array}{l}\text { Hand (mean metacarpal } \\
\text { length of phalanges 2-5) }\end{array}$ & $0.085 \pm 0.009$ & $4.9 \pm 0.5$ \\
\hline First metacarpal & $0.046 \pm 0.009$ & $2.7 \pm 0.5$ \\
\hline First proximal phalanx & $0.0369 \pm 0.004$ & $2.1 \pm 0.3$ \\
\hline First distal phalanx & $0.0276 \pm 0.004$ & $1.6 \pm 0.2$ \\
\hline Second proximal phalanx & $0.046 \pm 0.005$ & $2.7 \pm 0.2$ \\
\hline Second middle phalanx & $0.028 \pm 0.003$ & $1.6 \pm 0.1$ \\
\hline Second distal phalanx & $0.023 \pm 0.002$ & $1.3 \pm 0.1$ \\
\hline Third proximal phalanx & $0.048 \pm 0.009$ & $2.8 \pm 0.5$ \\
\hline Third middle phalanx & $0.033 \pm 0.004$ & $1.9 \pm 0.2$ \\
\hline Third distal phalanx & $0.024 \pm 0.002$ & $1.4 \pm 0.1$ \\
\hline Forth proximal phalanx & $0.043 \pm 0.010$ & $2.5 \pm 0.0$ \\
\hline Forth middle phalanx & $0.031 \pm 0.005$ & $1.8 \pm 0.2$ \\
\hline Forth distal phalanx & $0.023 \pm 0.002$ & $1.3 \pm 0.0$ \\
\hline Fifth proximal phalanx & $0.035 \pm 0.008$ & $2 \pm 0.4$ \\
\hline Fifth middle phalanx & $0.023 \pm 0.006$ & $1.3 \pm 0.3$ \\
\hline Fifth distal phalanx & $0.012 \pm 0.003$ & $1.1 \pm 0.2$ \\
\hline
\end{tabular}

\section{Acknowledgments}

The authors wish to thank Drs. Robert Gaunt, Lee Fischer, and Anton Sobinov for their contributions to the discussion of analysis in this study. 


\section{Additional Model Development}

As previously described, the state of muscle lengths determines muscle force production due to the force-length relationship. However, it is torque that ultimately acts around a joint and generates movement. Torque generation relates to not only the muscle forces but also muscle paths. Similar to muscle lengths, muscle moment arms are posture dependent. For example, as the elbow is flexed, the distance between the elbow joint and the biceps muscle increases, which in turn increases the torque that is generated with the same amount of muscle force. Therefore, musculoskeletal models must have accurate representation of muscle geometry to properly approximate the mechanical interactions of musculoskeletal system and torque generation. The following sections will briefly describe our process for validating moment arms in the musculoskeletal model and the current state of this model development.

\section{Moment Arm Validation}

We evaluated the anatomical accuracy of our musculoskeletal model by comparing simulated musculotendon moment arms with published measurements from the literature (Ackland et al., 2008; Quental et al., 2012; Folgado et al., 2013; Boots et al., 2020). Moment arms were simulated using the OpenSim software. A physiological coordinate system was added to the shoulder joint to allow comparison of simulated moment arms to values measured in the literature (see Shoulder DOFs). Each DOF was permuted across the defined range of motion uniformly at a sampling rate of 9 postures per DOF. Musculotendon moment arms were acquired for each DOF that a muscle crossed for each iteration (Fig. 2-8). Moment arm profiles were then interpolated using a polynomial fitting procedure described in Sobinov, 2019 (Sobinov et al., 2019). These polynomials approximated the musculotendon moment arms as a function of model posture. 


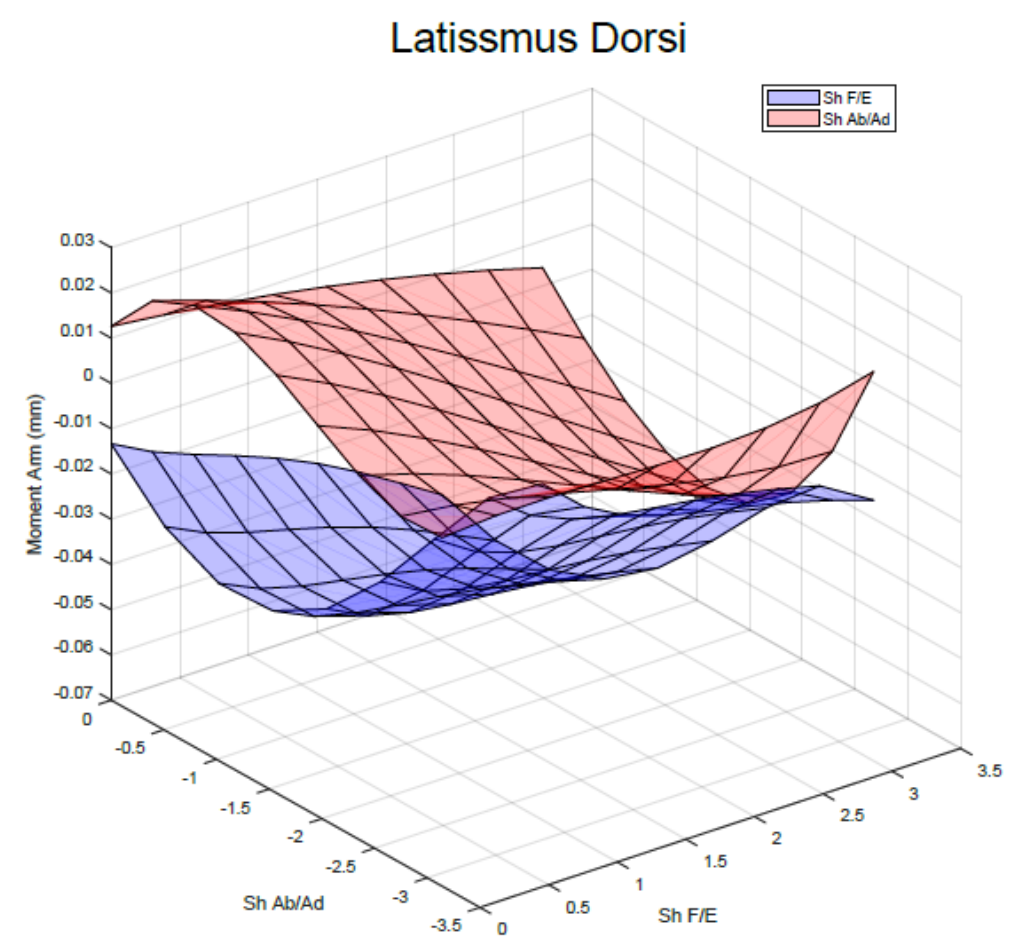

Figure 2-8: Example of changes in moment arms as a function of posture

To validate the physiological accuracy, we evaluated the polynomial approximations using the same postures that were used during moment arm measurements in the literature (Ackland et al., 2008; Quental et al., 2012; Folgado et al., 2013). The simulated moment arms were then compared to the published measurements by calculating the root mean square (RMS) normalized to the range of motion. Additionally, moment arm profiles were qualitatively assessed by classifying discordant moment arm profiles based upon the error that was induced. Boots et al, 2020 provides a more comprehensive description of the validation procedure along with results for the elbow, wrist, and hand (Boots et al., 2020). The remainder of this chapter will describe the validation of muscles spanning the shoulder.

\section{Shoulder DOFs}

The shoulder joint consists of three bony segments, each of which are comprised of three rotational and three translational DOFs (Fig. 2-9). The complex movement of these segments influence the dynamics of muscles spanning the shoulder because they alter 
and direct the muscle paths in a posture-dependent manner. Therefore, to accurately represent muscle lengths and moment arms, it is necessary to adequately emulate the behavior of all three of these segments across their respective range of motion.



Figure 2-9: Coordinates of the shoulder joint.

The local coordinate systems of the three bony segments that comprise the shoulder joint are shown. Each body can move along 3 rotational DOFs and 3 translational DOFs.

The Saul model previously addressed this complexity by applying several kinematic constraints which would drive the movement of the scapula and clavicle based upon the elevation of the humerus, such that $\left[\theta_{\text {scapula }}, \theta_{\text {clavicle }}\right]=f\left(\theta_{\text {humerus }}\right)$ (Saul et al., 2015b). To implement this method, a non-Euler set of coordinate rotations were used to accommodate the limitations of these constraints. To validate the musculotendon paths of shoulder muscles to values published in the literature, the shoulder coordinates were first transformed into the Euler coordinates (flexion/extension, abduction/adduction, internal/external rotation). The simulated moment arms were acquired as previously described using the non-Euler coordinate system to articulate the joint. The matrix of postures was then transformed into the Euler defined coordinate system prior to polynomial fitting. This transformation was achieved by first converting the model's axis- 
angle representation into a quaternion representation. The coordinates were then rotated using the Hamilton product.

$$
q^{\prime}=q_{1} q_{2}
$$

Here $q_{1}$ and $q_{2}$ are the first and second rotation respectively. Finally, the newly rotated quaternion coordinate was converted into Euler angles corresponding to flexion/extension, abduction/adduction, and internal/external rotation.

Moment arm profiles were plotted relative to the reference dataset created from the published values. Then, the differences between published and simulated moment arm values were iteratively changed by modifying muscle path in OpenSim to reduce the discrepancy (Fig. 2-10).
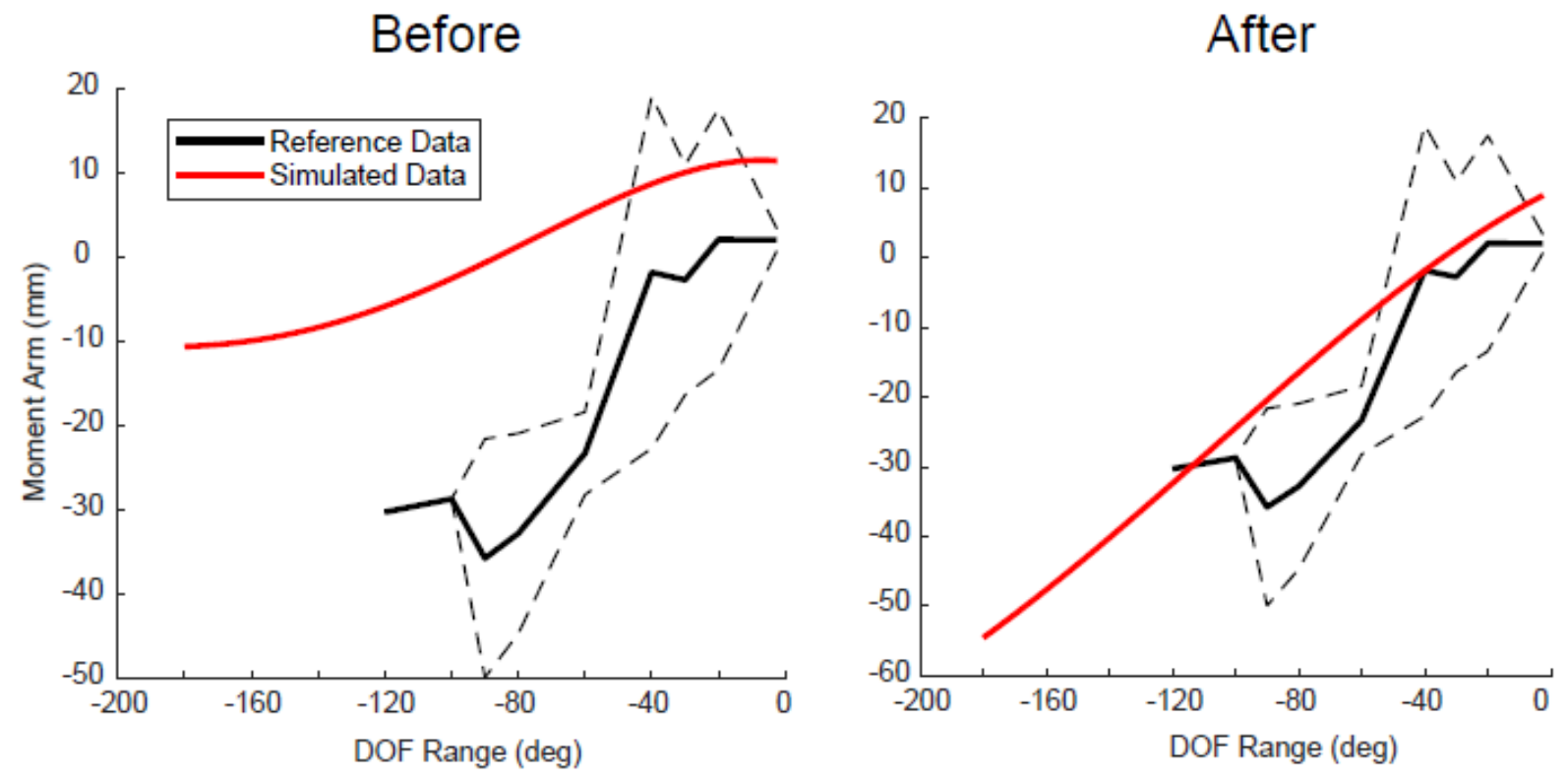

Figure 2-10: Example of moment arm validation.

The development and validation of shoulder muscle moment arms is ongoing, but the current status of the model is described using the aforementioned qualitative metric in Fig. 2-11. Moment arms around internal and external rotation are not included as experimental measurements have not yet been found in the literature. 

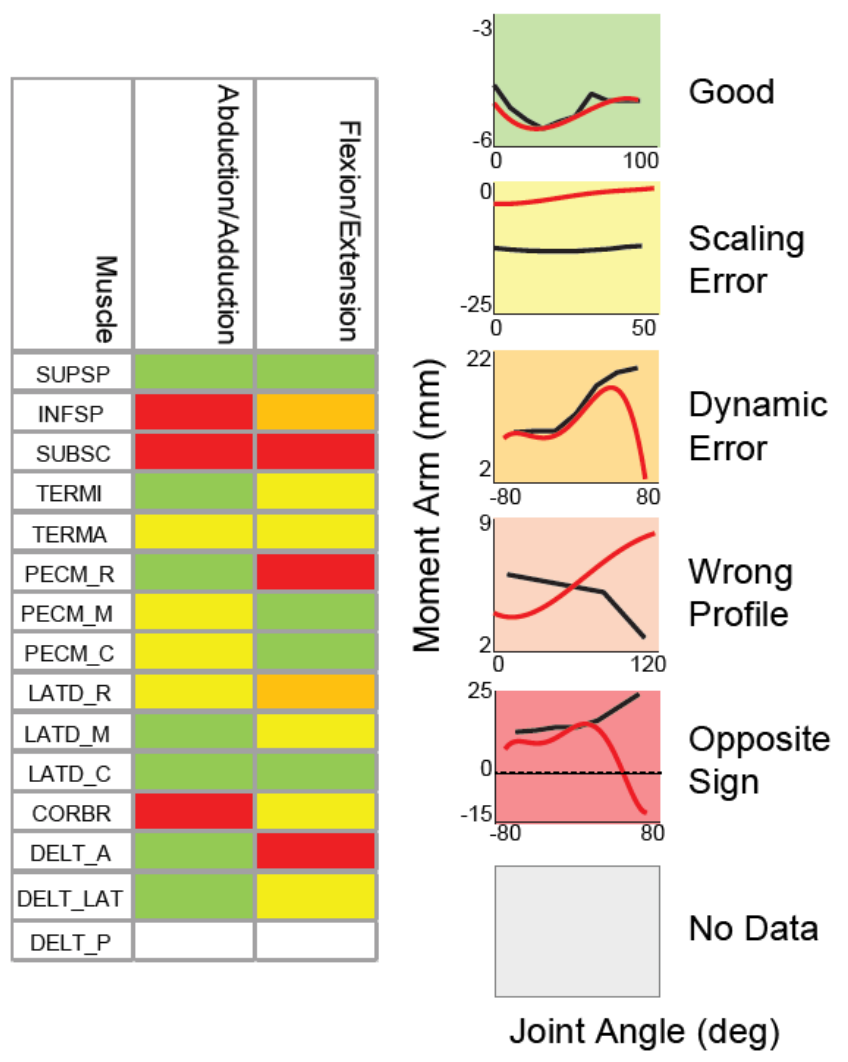

Figure 2-11: Qualitative assessment of moment arm quality.

\section{Preliminary Results}

The validated musculotendon moment arms were analyzed using the hierarchical clustering of HVE previously described in Methods. Shoulder muscle moment arms cluster into two distinct clusters based upon their action around each DOF (Fig. 2-12). This result is consistent with moment arm relationships at the elbow, wrist, and hand (Boots et al., 2020). The correlation of muscle moment arms with similar function means that moment arms change together in different postures, they do so as a functional group, e.g. if the moment arm of the anterior deltoid in increasing so too are the moment arms of other shoulder flexors. It is noteworthy that these clusters are not conserved across 
DOF, i.e. a shoulder flexor is not always an abductor nor is a shoulder extensor. Further model validation will confirm the robustness of these relationships. 

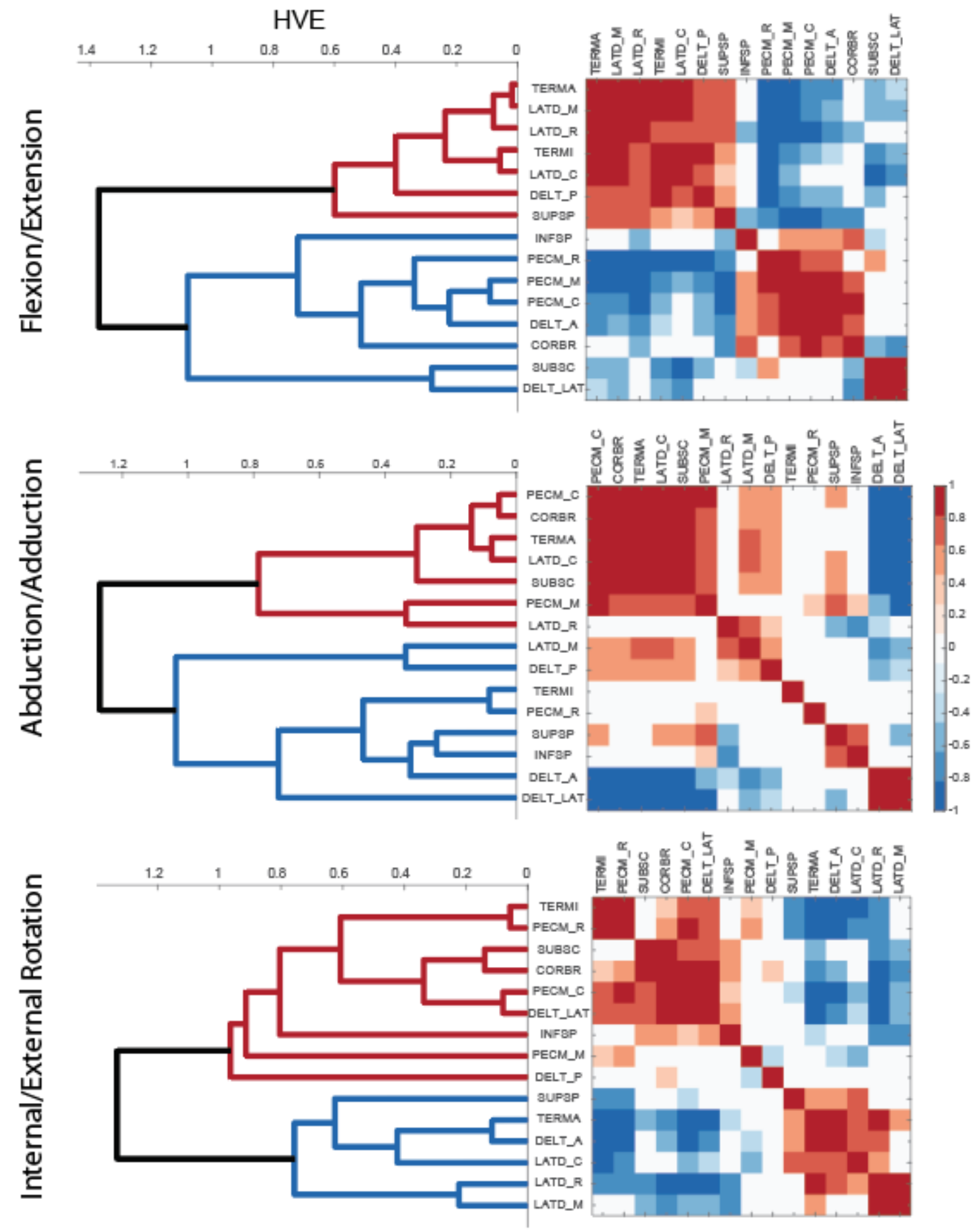

Figure 2-12: Hierarchical clustering of shoulder muscle moment arms.

Shoulder muscle moment arms cluster as distinct agonist-antagonist pairs. 


\section{Chapter 3 - Computational evidence for nonlinear feedforward modulation of fusimotor drive to antagonistic co-contracting muscles}

(this chapter has been directly adapted from Hardesty RL, Boots MT, Yakovenko S, Gritsenko V (2020) Computational evidence for nonlinear feedforward modulation of fusimotor drive to antagonistic co-contracting muscles. Scientific Reports 10:10625.)

\section{Abstract}

The sensorimotor integration during unconstrained reaching movements in the presence of variable environmental forces remains poorly understood. The objective of this study was to quantify how much the primary afferent activity of muscle spindles can contribute to shaping muscle coactivation patterns during reaching movements with complex dynamics. To achieve this objective, we designed a virtual reality task that guided healthy human participants through a set of planar reaching movements with controlled kinematic and dynamic conditions that were accompanied by variable muscle co-contraction. Next, we approximated the la afferent activity using a phenomenological model of the muscle spindle and muscle lengths derived from a musculoskeletal model. The parameters of the spindle model were altered systematically to evaluate the effect of fusimotor drive on the shape of the temporal profile of afferent activity during movement. The experimental and simulated data were analyzed with hierarchical clustering. We found that the pattern of co-activation of agonistic and antagonistic muscles changed based on whether passive forces in each movement played assistive or resistive roles in limb dynamics. The reaching task with assistive limb dynamics was associated with the most muscle co-contraction. In contrast, the simulated la afferent profiles were not changing between tasks and they were largely reciprocal with homonymous muscle activity. Simulated physiological changes to the fusimotor drive were not sufficient to reproduce muscle co-contraction. These results largely rule out the static set and $\alpha-\gamma$ coactivation as the main types of fusimotor drive that transform the monosynaptic la afferent feedback into task-dependent co-contraction of antagonistic muscles. We speculate that another type of nonlinear transformation of la afferent signals that is 
independent of signals modulating the activity of a motoneurons is required for la afferentbased co-contraction. This transformation could either be applied through a complex nonlinear profile of fusimotor drive that is not yet experimentally observed or through presynaptic inhibition.

\section{Introduction}

Movement is the product of interactions between neural signals and the musculoskeletal dynamics that depends on limb anatomy(Nishikawa et al., 2007a; Prochazka and Yakovenko, 2007b; Yakovenko, 2011; Gritsenko et al., 2016). The motor control problem is then solved within a system with coupled neural and mechanical dynamical elements(Schöner and Kelso, 1988; Taga et al., 1991b; Ting et al., 2015a). Therefore, the relationship between neural signals driving muscle contraction and the resulting motion is nonlinear. Muscle contractions generate forces that sum into active moments defined by the agonistic or antagonistic relationships between the muscle's moment arms around a given axis of rotation of the joint. The components of these forces that sum to zero moment, such as forces produced by balanced co-contraction of antagonistic muscles, define joint stiffness and viscosity. The remaining unbalanced moments are often termed muscle torques; they produce motion. In this bottom-up reasoning, muscle contractions represent the output of the central nervous system (CNS) that also reflects the mechanical properties of the limb being moved by these muscles. For example, muscle torques derived from motion capture share a large amount of variance with muscle activity profiles during reaching movements in certain directions(Olesh et al., 2017b), while in other directions co-contraction defines the muscle activity profiles more than muscle torques. Joint stiffness is the product of co-contraction of antagonistic muscles. However, without the knowledge of the moment arms and motor unit recruitment of these muscles, it is often difficult to estimate experimentally joint stiffness from surface electromyography. Some studies estimate co-contraction using "wasted contraction", i.e. the minimal value of estimated muscle recruitment between antagonistic muscles(Thoroughman and Shadmehr, 1999; Gribble et al., 2003; Darainy and Ostry, 2008). Other studies measure joint stiffness more directly with perturbations(De Serres and Milner, 1991; Damm and Mclntyre, 2008; Wong et al., 2009). 
These studies found that both co-contraction and stiffness change with task demands, so that the co-contraction increases in the novel, precise, or demanding tasks and the resulting stiffness depends on limb dynamics. The joint and, possibly, whole arm stiffness is thought to be a controlled parameter in the CNS ensuring movement stability(Milner and Franklin, 2005; Franklin et al., 2007). An important question in motor control is how the co-contraction of antagonistic muscles that modulate stiffness with stability is produced.

It is well established that at the lowest level of the CNS, the primary afferents (la) from muscle spindles can increase the activity of the homonymous muscle and its agonists, thus increasing their stiffness, through the homonymous monosynaptic reflex(Angel et al., 1996). It is also well established that the same la feedback through an interneuron can inhibit the activity of the antagonistic muscle, contributing to the reciprocal muscle activation during locomotion(Hongo et al., 1966; Lundberg, 1969). The contribution of these pathways to muscle activation can be modulated via the activity of dynamic and static $\gamma$ motoneurons that change the profile of activity of the la afferent(Boyd, 1985). During movement, the dynamic fusimotor action changes mainly the velocity sensitivity of the la afferents, while the static fusimotor action changes mainly the length sensitivity of the la afferents(Matthews, 1959b; Prochazka, 2011). How exactly the activity of $\gamma$ motoneurons changes during reaching movements in humans is unknown (for the reviews of afferent recording studies see(Prochazka, 2011; Macefield and Knellwolf, 2018)). However, the effect the fusimotor drive has on shaping the muscle spindle output can be broadly classified based on whether the fusimotor drive is constant or changing during movement. The former is defined as the static set, where $\gamma$ motoneuron activity remains constant during a given movement, but its level changes between different movement types adjusting muscle spindle sensitivity to the anticipated demands of the task(Prochazka et al., 1985; Prochazka, 1986). Alternatively, the la feedback could be coupled to the ongoing motor activity via $\alpha-\gamma$ coactivation, where the sensitivity of muscle spindles is maintained during muscle shortening by coupling the activity of $\gamma$ motoneurons to the activity of $\alpha$ motoneurons(Granit, 1970; Hagbarth, 1993). The fusimotor drive provided by $\beta$ motoneurons, which innervate both extrafusal and intrafusal muscle fibers(Kakuda et al., 1998), can also modulate la afferent activity. 
However, in our correlative study, the effect of $\beta$ motoneurons is indistinguishable from the effect of $\alpha-\gamma$ coactivation. Given such complex and flexible la feedback that could be transmitted through the mono- and disynaptic pathways, the role that it plays in cocontraction and ultimately limb stiffness is unknown. It has been suggested the cocontraction of antagonistic muscles can be modulated by descending signals through the concurrent fusimotor drive, e.g. C command in lambda-model(Feldman, 1966). A pathological change in the strength of the monosynaptic connection of la afferents to $\alpha$ motoneurons is also implicated in spasticity, a condition that is characterized by abnormal co-contraction of antagonistic muscles(Brown, 1994). The question arises whether the common fusimotor drive to muscle spindles in antagonists can contribute significantly to their co-contraction through the monosynaptic la feedback under normal conditions, such as during reaching. Answering this question will help constrain the space of possible solutions for descending neural control signals.

The current methods of directly observing primary afferent firing in humans, such as microneurography, are limited in the number of observable signals and the types of behaviors these observations can be made under. In presence of these limitations, the experimentally validated models of primary afferents(Prochazka and Gorassini, 1998) and the musculoskeletal anatomy of the arm(Saul et al., 2015a) used together can provide unique insight into the transformation through the motoneuron pool. The computational approach enables a holistic computational estimation of the la afferent activity from multiple muscles during reaching movements in humans. Here, we used the model of muscle spindle with the two types of fusimotor drive, static set and $\alpha-\gamma$ coactivation, to address the question of la afferent contribution to the co-contraction of multiple muscles during reaching movements. The movements were selected based on the roles of passive forces, assistive or resistive, during reaching that were expected to be accompanied by different patterns of muscle co-contraction. We then used a mathematical model of la afferent with two parameters that define the sensitivity of muscle spindle to muscle length and velocity changes(Prochazka and Gorassini, 1998). We changed these parameters across tasks according to the experimental observations that informed the two types of fusimotor drive. We took advantage of the linearizing properties of the motoneuron pool in transforming synaptic drive into neural command to the 
muscles(Farina et al., 2014). We used electromyography (EMG) to estimate the ensemble activity of the motoneuron pool(Hoffer et al., 1987; De Luca and Hostage, 2010; Farina et al., 2014). During reaching without heavy objects, the maximal EMG in arm muscles is estimated to be low, $5-10 \%$ of maximal voluntary contraction (Tagliabue et al., 2015; Aurbach et al., 2020). At that range, the relationship between EMG and the recruitment of motoneuron pool is largely linear (De Luca and Hostage, 2010). This justified employing a hierarchical clustering analysis to quantify the linear relationships between time-varying muscle activity (EMG), including co-contraction, and simulated la afferent activity. We expected that the activity of co-contracting antagonistic muscles will positively correlate with the activity of their la afferents shaped by static set and $\alpha-\gamma$ coactivation, which would be evident from observing these signals in the same clusters.

\section{Materials and Methods}

Experimental design and human participants

We recruited 9 healthy adults ( 5 males, 4 females; age, $24.3 \pm 1.8$ years; weight, $76.3 \pm 14.5$ kilograms) to perform reaching movements to visual targets in a virtual reality (VR, Oculus Rift, developer kit 2). All procedures were approved by the West Virginia University Institutional Review Board (IRB). All methods were performed in accordance with the IRB guidelines and regulations; informed consent was obtained from all individuals prior to their participation in the study. All data analysis and simulations were performed in Matlab (MathWorks, RRID:SCR_001622). 

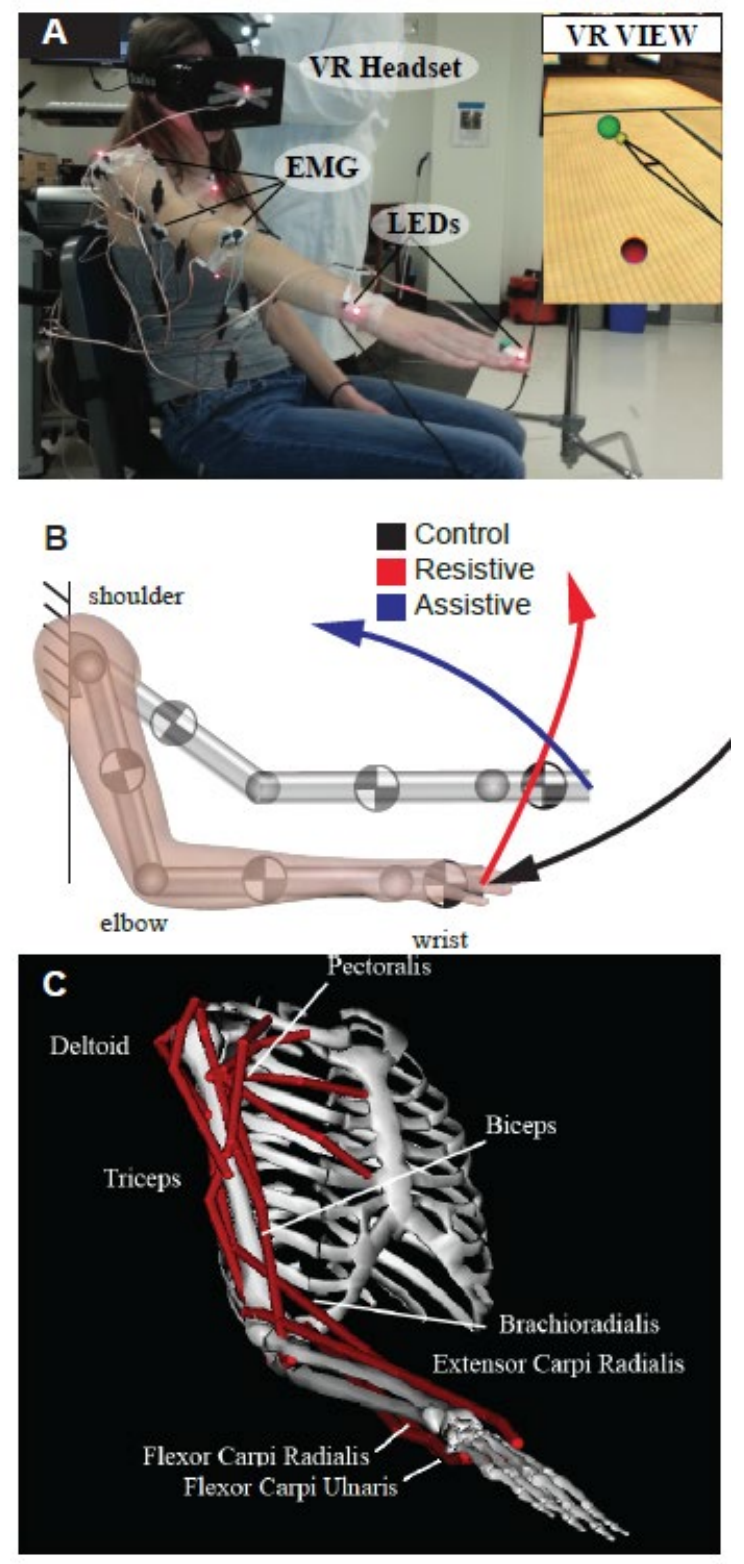

Figure 3-1: Illustrations of the experimental setup and arm models.

Oculus DK1 is shown, but all data collection occurred with Oculus DK2 headset. A. Annotated photo of the setup; insert shows participant's monocular view. Reaching target is in green, origin target is in red. Yellow sphere shows the location of individual's fingertip and the black lines outline the major arm segments for visual feedback of arm location in VR. B. Colored lines show the fingertip trajectories of each of the three tasks. Arrows indicate the direction of motion toward the reaching target. The grey blocks show the locations and orientations of local coordinate systems used to obtain joint torques from motion capture. Circles with black and white quarters indicate the locations of the centers of mass and the orientations of local coordinate systems. C. Illustration of the OpenSim model used to derive muscle lengths for the calculations of la afferent discharge. Red lines show the anatomical paths of each muscle from which EMG signals were recorded during experiments. 
Participants performed three reaching tasks in VR (Fig. 3-1A). Pairs of visual targets defined the starting and goal target locations for each task (Fig. 3-1B). The virtual environment provided two distinct advantages: 1 ) it allowed target locations to be quickly calculated and scaled based upon an individual participant's proportions and 2) it provided visual guidance to constrain movement trajectories without physically interacting with the participant, i.e. altering limb dynamics. To minimize inter-subject variability in angular kinematics, the locations of virtual targets were derived using planar trigonometry based on the lengths of individual's arm and forearm segments and displayed relative to the subject's shoulder location in VR. This resulted in the same shoulder, elbow, and wrist angles at the start and end of each movement across participants. The pairs of starting and goal visual targets were shown in a random sequence to minimize bias. The cue to move was the change of target color from red to green. Trunk motion was restricted with straps, wrist was instructed to be kept at neutral palm down (Fig. 3-1A). Each task was repeated 24 times. At the beginning of each 60 -trial block, the virtual target positions were re-calibrated to the participant's shoulder location.

The tasks were based on planar pointing movements selected for their diverse dynamical contexts. The Control movement (Fig. 3-1B, black) was largely passive with the arm being lowered with gravity. The Resistive movement (Fig. 3-1B, red) was accompanied by increasing gravitational load at the shoulder and resistive interaction torques between the shoulder and elbow(Gritsenko et al., 2011b). Finally, the Assistive movement (Fig. 3-1B, blue) was accompanied by decreasing gravitational load at the shoulder and assistive interaction torques between the shoulder and elbow. The dynamical contexts were identified based on inverse simulations in Simulink (RRID:SCR_014744) with a mechanical planar model of the arm(Olesh et al., 2017b) that predicted shoulder and elbow torques for a given linear trajectory between an arbitrary set of starting and ending postures.

During the performance of each task, we recorded the kinematics of the shoulder, elbow, and wrist joints and electromyography (EMG) of 12 muscles that span those joints. The recorded muscles were the anterior and posterior deltoids ( $A D$ and PD, respectively), pectoralis major (Pec), teres major (TM), biceps brachii long and short heads (BicL and BicS, respectively), triceps brachii lateral and long heads (TriLa and TriLo, respectively), 
brachioradialis $(\mathrm{Br})$, extensor carpi radialis $(\mathrm{ECR})$, flexor carpi radialis $(\mathrm{FCR})$, and flexor carpi ulnaris (FCU). These muscle abbreviations are used consistently throughout the manuscript and figures. Motion capture data were recorded at 480 hertz $(\mathrm{Hz})$ using an Impulse system (PhaseSpace), and EMG signals were recorded at $2000 \mathrm{~Hz}$ with an MA400-28 system (MotionLab Systems). Nine LED markers were placed on bony landmarks of the arm and trunk (Cervical Vertebrae 7, Xiphoid Process, Sternoclavicular Joint, Acromial Edge, Acromioclavicular Joint, Lateral Olecranon Process, Radioulnar Joint, Styloid Process, and the Distal Phalanges Head). The start and end of each movement was defined by finding a local maximum in the $3^{\text {rd }}$ derivative of the vector distance profile of the wrist and elbow LED markers. The motion capture data were used to derive joint angles by fitting local coordinate systems into the markers defining each major segment and deriving Euler angles between them using linear algebra(Robertson et al., 2013b). The EMG was processed consistent with SENIAM recommendations, it was high-pass filtered at $10 \mathrm{~Hz}$, rectified, and low-pass filtered at $20 \mathrm{~Hz}$. The resulting EMG profiles were time-normalized between onset and offset of each movement, averaged per task, and amplitude-normalized to the maximum across all tasks per participant. Co-contraction was calculated as "wasted contraction"(Thoroughman and Shadmehr, 1999; Gribble et al., 2003; Darainy and Ostry, 2008) between normalized EMG profiles of pairs of antagonists defined as follows, AD-PD, Pec-TM, BicL-TriLo, BicS-TriLa, Br-TriLa, FCR-ECR, and FCU-ECR.

\section{Primary afferent model}

To estimate the sensory contribution from muscle spindles during movement, we used Prochazka's model of primary afferent discharge(Prochazka, 1999), which offers a clear parametrization of static and dynamic responses. The spindle model relates afferent firing rate $(l a)$ to the time-varying muscle length $(I)$ and its rate of change $(v)$ as follows:

$$
I a(v, l)=A v^{0.5}+B l+C
$$

where the constant parameters $(A=65, B=200$, and $C=10)$ were validated empirically to reflect human microneurography data(Malik et al., 2016).

The changes of musculotendon length during movement were calculated in OpenSim (RRID:SCR_002683) using a modified musculoskeletal model of the human 
arm(Saul et al., 2015a) (Fig. 3-1C). This model was adjusted for each individual using segment lengths to scale model's segments and move proportionally the origin and insertion of each simulated muscle. Muscle lengths were simulated by driving the adjusted model with the mean angular trajectories for each task and participant. This resulted in temporal profiles of muscle length $(l)$ in units of meters and its derivative $(v)$ in units of meters per second for each movement per participant. Muscle lengths profiles used in the la model were converted to the rest-length units based on the minimal and maximal muscle lengths observed across all the possible postures of the OpenSim model in Gritsenko et al.(Gritsenko et al., 2016). The rest length was defined as half the length between the maximal and minimal muscle length values(Yakovenko et al., 2004). The muscle shortening/lengthening velocity profiles used in the la model were converted to the rest length per second units. The parameter space of $A$ and $B$ variations was explored in the context of variable fusimotor drive. To simulate a change in the dynamic fusimotor drive, we varied the velocity coefficient $A$; to simulate a change in the static fusimotor drive, we varied the length coefficient $B$. The following parameter ranges were explored: $A \in[33200]$ and $B \in[50400]$, which resulted in 4 models of static set referred to below as follows: V33-L50, V33-L400, V200-L50, V200-L400, where V stands for velocity coefficient and $\mathrm{L}$ stands for muscle length coefficient. This also served as a sensitivity analysis of the two parameters of the la model.

Separately, we approximated $\alpha-\gamma$ coactivation that affects both the dynamic and static fusimotor drive using EMG profiles which transformed equation (1) as follows:

$$
I a(a, v, l)=a \cdot 65 v^{0.5}+a \cdot 200 l+10
$$

where $a$ is the normalized mean EMG profile for a given task and participant. The model in (2) is referred to below as EMG-coupled la model.

The time-varying primary afferent profiles calculated with Eq. (1) and (2) are referred to below as la profiles. For the regression analysis described below, the la profiles were amplitude-normalized to the maximum across all tasks per participant to obtain unitless values. 
Verifying task dynamics


Figure 3-2: Signals calculated from motion capture.

Thick lines show normalized mean trajectories for each movement across all participants, shaded areas show standard deviations across participants. Only rotatum signals calculated for shoulder flexion/extension degree of freedom included in the following analyses are shown. Movement phase represents normalized duration of each movement with 0 indicating the start of movement (vertical onset line) and 1 indicating the end of movement. A. joint angles; B. joint angular velocity; C. muscle torques.

A mechanical model of a human upper-limb(Olesh et al., 2017b) was used to compute joint torques from joint angles inferred from motion capture. The mechanical model described above was expanded to comprise three segments and five degrees of freedom, including the shoulder (flexion/extension, abduction/adduction, internal/external rotation), elbow (flexion/extension), and wrist (flexion/extension). The height and weight of each individual were used with anthropometric tables(Winter, 2009a) to estimate the lengths and cylindrical inertias of the arm, forearm, and hand segments (Fig. 3-1B). To calculate active torques that result from muscle action, mean angular trajectories from each individual and task were used to drive the subject-specific model in inverse dynamic simulations (Fig. 3-2). The motion defined by our tasks was in the vertical plane. Participants showed minimal out-of-plane motion as measured by angular trajectories about shoulder abduction/adduction and internal/external rotation degrees of freedom. 
Therefore, only muscle torques about the shoulder flection/extension degree of freedom was included in the analysis described below.

To describe the limb dynamics of each task, the three muscle torques about shoulder, elbow, and wrist were then used to calculate the following parameters. 1) The postural torque change was calculated as the difference between muscle torques averaged over $100 \mathrm{~ms}$ prior to onset and following the offset of movement. These postural torques are produced to maintain the arm in starting and final postures against the force of gravity. 2) The peak torque change in acceleration phase was calculated as the maximal change in torque between the start of movement to its halfway point. The first half of movement was used to ascertain the amount of muscle force that is required to start the motion, thought to represent largely feedforward activation. 3) The mechanical muscle work was calculated by integrating a product between muscle torques and angular velocity as described in(Winter, 2009a). When the direction of action matches between muscle torque and angular velocity, as indicated by the same sign (both positive or both negative), the mechanical muscle work is positive. This means that agonist muscle contractions about the corresponding degree of freedom are concentric and they are actively producing the motion. When the direction of action is opposite between muscle torque and angular velocity, as indicated by opposite signs, the mechanical muscle work is negative. This means that agonist muscle contractions about the corresponding degree of freedom are eccentric and the motion is produced by passive torques, such as gravity, interaction torques, etc. In our tasks, the wrist joint does not move, therefore the mechanical muscle work about the wrist is zero. This means that the muscle torque about the wrist reflects the isometric contraction of wrist and hand muscles that is required to stabilize the joint at a constant angle.

Analysis

To quantify the common and distinct features in EMG and la profiles and to compare them to features obtained from muscle lengths we used hierarchical clustering. The relationships between the normalized averaged EMG and la profiles were characterized by a matrix of Pearson's correlation coefficients $(r)$. To reduce the probability of Type I errors, the $\alpha$ for determining the significance of $r$ values was adjusted using the two-stage Benjamini, Krieger, and Yekutieli procedure for controlling false detection rate(Benjamini 
et al., 2006). The correlation matrix was then transformed into the heterogeneous variance explained (HVE) as follows:

$$
H V E=\left\{\begin{array}{c}
1-r^{2}, \mid r>0, p<\alpha \\
1+r^{2}, \mid r<0, p<\alpha \\
1, \mid p \geq \alpha
\end{array}\right.
$$

The HVE transforms the large positive $r$ values that are characteristic of agonistic relationships into short distances close to 0 and the large negative $r$ values corresponding to antagonistic relationships into long distances close to 2. To identify synergistic relationships between EMG and la, we applied hierarchical clustering to an unbiased HVE distance matrix using the linkage function with an unweighted average distance method(Gritsenko et al., 2016). The goodness of fit of the clustering model was assessed using the cophenetic correlation coefficient, which quantified how faithfully the hierarchical cluster tree represented the dissimilarities among observations. The magnitude of this value should be very close to 1 for a high-quality solution. As a result of this analysis, the strongly and positively correlated signals will be labeled belonging to the same cluster, and we will be able to assess the degree of similarity between these clusters based on the strength of the positive and negative correlations between them. This approach is advantageous in examining the correlation structure while still distinguishing between positive and negative correlations. Hierarchal cluster analysis has captured the relationship between EMG and la signals with high precision, as evidenced by high cophenetic coefficient of $0.81 \pm 0.044$, mean and standard deviation across participants.

Clusters were compared using the Fowlkes-Mallows index $\left(B_{k}\right)$ to assess cluster similarity between separate hierarchical cluster trees(Fowlkes and Mallows, 1983). The Fowlkes-Mallows index represents a normalized number of common elements between clusters from different trees at the same cluster height. For example, $B_{2}$ indicates that the hierarchical trees were compared at the height, where only 2 clusters occur. Here, we explored $k=[2, \ldots, n]$, where $n$ is half the number of signals being included in the 
hierarchical clustering. Thus, for two cluster trees with arbitrarily numbered clusters $i=1$, $\ldots, k$ and $j=1, \ldots, k$ we can use the number of objects between the ith cluster of one tree and $j$ th cluster of the other tree $\left(m_{i j}\right)$ to calculate the index as follows:

$$
B_{k}=\frac{T_{k}}{\sqrt{P_{k} \cdot Q_{k}}},
$$

where

$$
\begin{gathered}
T_{k}=\sum_{i=1}^{k} \sum_{j=1}^{k} m_{i j}^{2}-n, \\
P_{k}=\sum_{i=1}^{k}\left(\sum_{j=1}^{k} m_{i j}\right)^{2}-n, \\
Q_{k}=\sum_{j=1}^{k}\left(\sum_{i=1}^{k} m_{i j}\right)^{2}-n,
\end{gathered}
$$

At each cluster division, the index is calculated such that $0 \leq B_{k} \leq 1$, where $B_{k}=1$ indicates two identical clusters and provides a means to compare the multi-muscle clustering of EMG and la signals. A further benefit of the Fowlkes-Mallows index is that it approaches 0 with an increasing number of data points, making it less sensitive to spurious correlations than the commonly used Rand index (Fowlkes and Mallows, 1983).

In an earlier study(Gritsenko et al., 2016), we quantified the synergistic relationships between muscles based on their anatomy using the same musculoskeletal model of the arm used here for muscle length measurements. The muscle lengths were calculated over the whole range of physiological joint postures and analyzed using the same hierarchal clustering method described above. Here we selected a subset of muscles recorded in this study and compared the clustering structure of the muscle lengths across all postures in Gritsenko et al.(Gritsenko et al., 2016) study to the clustering structure of simulated la afferent activity. We used the muscle lengths obtained from Gritsenko et al.(Gritsenko et al., 2016) rather than the muscle lengths calculated for the la modeling, 
because the former was calculated over a wider range of postures than the latter. Because the muscle lengths data is part of the Eq. (1), similar clustering structure is expected between muscle lengths and la profiles. These muscle length data were used as one of the controls for the statistical analysis of clustering structure described below.

\section{Statistics}

All values reported in results are means with standard deviations across participants, unless stated otherwise. The shared variance $\left(R^{2}\right)$ between clusters defined by hierarchical clustering was assessed using t-tests. The t-tests were applied to $R^{2}$ values averaged across members of the cluster per participant per task. Individuals were assumed to represent independent samples. The combined $p$-values across participants included in the tables were obtained using the Fisher's combined probability test(Fisher, 1970). Correction for multiple testing was based on Bonferroni adjustment of alpha, the acceptable probability of making type I error(Dunn, 1959).

The statistical comparison of hierarchical clustering between multiple signal modalities was based on permuting the hierarchical clustering trees to estimate the chance of observing spurious correlations. The hierarchal tree for each participant each movement type and each signal modality (la, EMG, muscle length) was randomly permuted 1000 times. Then the Fowlkes-Mallows index $(B)$ was calculated between each of the permuted trees, which resulted in a population of $B$ values that represents the distribution of noise. The distribution of experimental $B$ values across tasks and individuals was compared to the corresponding noise distribution of $B$ values to test the hypothesis that $B_{\text {experimental }} \neq B_{\text {noise. }}$. The $\mathrm{p}$-value for each experimental $\mathrm{B}$ value was determined from the corresponding noise distribution for each individual using the percentile method(Efron and Tibshirani, 1994). The combined p-values across cluster subdivisions included in the supplementary tables were obtained using the Fisher's combined probability test(Fisher, 1970). The significant alpha was set to 0.0056 to adjust for repeating tests across 9 participants. This permutation analysis was applied to test three hypotheses. The $1^{\text {st }}$ hypothesis was that the similarity between la and muscle-length clusters is not spurious. This is a test of the chosen statistical method. We expect to support the $1^{\text {st }}$ hypothesis, because the la and muscle length profiles are not independent, i.e. the former is derived from the latter as described in Eq. 1 . The $2^{\text {nd }}$ hypothesis was 
that the similarity between la and EMG clusters is not spurious. The $3^{\text {rd }}$ hypothesis was that EMG and la clusters change the same way between tasks. Supporting either $2^{\text {nd }}$ or $3^{\text {rd }}$ hypothesis means that the compared trees are similar to a greater extent than is expected by chance, and that the afferent activity clusters comprise the same muscles as the muscles that co-activate in a given task.

The statistical comparison of hierarchical clustering between tasks was based on bootstrapping the $B$ values(Efron and Tibshirani, 1994). The $B$ values for EMG clustering $\left(B_{E M G}\right)$ and la clustering $\left(B_{l a}\right)$ calculated between tasks were resampled with replacement 1000 times. This resulted in two distributions of $45,000 B_{E M G}$ and $B_{l a}$ values for each task pair (Control-Resistive, Control-Assistive, and Resistive-Assistive). These data were used to test the $3^{\text {rd }}$ hypothesis that EMG and la clusters change the same way between tasks. To test this hypothesis, we calculated the difference between the two distributions of $B_{E M G}$ and $B_{l a}$ values across tasks, each comprising 1000 bootstraps per cluster number $(k=2, \ldots, 6)$ per participant $(\mathrm{N}=9)$. The $\mathrm{p}$-value for each task pair was determined from the location of the 0 value in the resulting distribution of differences, which indicated no difference between cluster structures, using the percentile method(Efron and Tibshirani, 1994).

The last set of hypotheses addressed the extent to which the fusimotor drive can shape la afferent discharge and capture muscle co-contraction. The hypothesis for each altered la model was that the similarity between la and EMG clusters is increased by alternative fusimotor drives. To test these hypotheses, the $B$ values were calculated between the hierarchical clustering of la and EMG profiles for each of the models with altered coefficients (V33-L50, V33-L400, V200-L50, V200-L400, and EMG-coupled). The distribution of $B$ values from each of the altered model was subtracted from the corresponding $B$ values based on la profiles from the Prochazka model. The $p$-value for each model with altered coefficients was determined based on the location of the 0 value in the resulting distribution of differences using the percentile method(Efron and Tibshirani, 1994). 


\section{Results}

Participants performed reaching tasks with consistent angular kinematics within the constraints defined by the VR targets. The angular excursions of each joint were similar across individuals for the three tasks (Fig. 3-2A), the angular velocity was most variable across participants in the Assistive task (Fig. 3-2B). Because the individual's movements were not restricted, most participants moved slightly out of the sagittal plane and the experimental angular displacement differed somewhat from those defined by virtual targets (Table 1). In the Control and Assistive tasks, the virtual targets defined joint excursions that required the shoulder and elbow joints to rotate in opposite directions. This caused assistive interaction torques between these joints similar to the Assistive task in Gritsenko et al.(Gritsenko et al., 2011b), which were associated with negative muscle work at the shoulder and positive muscle work at the elbow (Table 1). The sign of work indicates the direction of energy flow. The positive sign of work indicates concentric contractions that transfer energy from muscles to segments, while the negative sign of work indicates eccentric contractions during which the energy from external forces are overpowering the muscle action and doing the work(Winter, 2009a). Thus, in the Control and Assistive tasks, the shoulder motion was largely passive, and the activity of shoulder muscles was compensating for external forces due to gravity and interaction torques. In the Control task, elbow and wrist torques were the lowest across the three tasks (Fig. 32C, black lines). The Assistive task was accompanied by decreasing postural torques in all joints, low acceleration shoulder torques, but high deceleration elbow and wrist torques (Fig. 3-2C, blue lines; Table 1, third column). This shows that in the Assistive task most of the muscle action was to decelerate the limb accelerated primarily by the interaction torques and gravity. In contrast, in the Resistive the joint excursions were such that required the shoulder and elbow to rotate in the same direction, causing resistive interaction torques similar to the Resistive task in(Gritsenko et al., 2011b). Altogether, this caused the opposite pattern of shoulder torques compared to that in the Control and Assistive tasks, while maintaining the same elbow and wrist torques to that in the Assistive task (Fig. 3-2C). In the Resistive task, the mechanical muscle work was always positive, 
indicating that muscle contractions were concentric and that the motion was produced with the least reliance on passive limb dynamics and gravity.

Table 1. Table of task parameters.

\begin{tabular}{|c|c|c|c|}
\hline & Control & Resistive & Assistive \\
\hline $\begin{array}{l}\text { Shoulder target-defined excursion- } \\
\text { (deg) }\end{array}$ & -60 & -45 & -40 \\
\hline experimental excursion (deg) & $-48 \pm 2 E$ & $40 \pm 4 \mathrm{~F}$ & $-35 \pm 5 E$ \\
\hline postural torque change $(\mathrm{Nm})$ & $-2.7 \pm 0.3 \mathrm{~F}$ & $1.3 \pm 0.5 \mathrm{~F}$ & $-3.2 \pm 0.5 \mathrm{~F}$ \\
\hline $\begin{array}{l}\text { peak torque change in acceleration phase } 8 \\
(\mathrm{Nm} / \mathrm{s})\end{array}$ & $8.3 \pm 3.6 \mathrm{E}$ & $9.0 \pm 3.1 \mathrm{~F}$ & $4.5 \pm 1.8 \mathrm{E}$ \\
\hline mechanical muscle work $(\mathrm{J})$ & $-3.80 \pm 0.39$ & $3.05 \pm 0.42$ & $-2.60 \pm 0.23$ \\
\hline $\begin{array}{l}\text { Elbow target-defined excursion } 6 \\
\text { (deg) }\end{array}$ & 60 & 10 & 100 \\
\hline experimental excursion (deg) & $42 \pm 4 \mathrm{~F}$ & $7 \pm 3 \mathrm{~F}$ & $81 \pm 8 \mathrm{~F}$ \\
\hline postural torque change $(\mathrm{Nm})$ & $0.3 \pm 0.2 \mathrm{~F}$ & $-1.0 \pm 0.2 \mathrm{~F}$ & $-0.9 \pm 0.4 \mathrm{~F}$ \\
\hline $\begin{array}{l}\text { peak torque change in acceleration phase } 1 \\
(\mathrm{Nm} / \mathrm{s})\end{array}$ & $1.8 \pm 0.5 \mathrm{~F}$ & $5.2 \pm 2.3 \mathrm{E}$ & $5.3 \pm 3.4 \mathrm{E}$ \\
\hline mechanical muscle work $(\mathrm{J})$ & $0.99 \pm 0.20$ & $0.28 \pm 0.06$ & $1.93 \pm 0.24$ \\
\hline
\end{tabular}




\begin{tabular}{|c|c|c|c|}
\hline & Control & Resistive & Assistive \\
\hline $\begin{array}{ll}\text { Wrist } & \text { target-defined excursion } \\
(\text { deg }) & \end{array}$ & 0 & 0 & 0 \\
\hline experimental excursion (deg) & $0.7 \pm 4.3 \mathrm{~N}$ & $1.4 \pm 3.7 \mathrm{~N}$ & $7.5 \pm 13.3 \mathrm{E}$ \\
\hline postural torque change $(\mathrm{Nm})$ & $0.0 \pm 0.03 \mathrm{~N}$ & $-0.15 \pm 0.05 \mathrm{~F}$ & $-0.14 \pm 0.10 \mathrm{~F}$ \\
\hline $\begin{array}{l}\text { peak torque change in acceleration phase } \\
(\mathrm{Nm} / \mathrm{s})\end{array}$ & $0.3 \pm 0.2 \mathrm{E}$ & $0.8 \pm 0.3 \mathrm{~F}$ & $0.9 \pm 0.6 \mathrm{~F}$ \\
\hline mechanical muscle work $(\mathrm{J})$ & $-0.00 \pm 0.01$ & $0.00 \pm 0.01$ & $-0.01 \pm 0.03$ \\
\hline
\end{tabular}

All joint angles are around flexion/extension axes of rotation for the three major joints included in the analysis. The sign of angles and torques indicates the direction of change of the corresponding measure, positive for increase and negative for decrease. Experimental values are averages with standard deviations across participants. Target-defined excursion is different from the experimental excursion due to out-ofplane arm motion. $\mathrm{F}$ indicates flexion direction of action; $\mathrm{E}$ indicates extension direction of action; $\mathrm{N}$ indicates no change. Negative values of mechanical muscle work imply work done by external forces, e.g. gravity and reaction from distal segments in the mechanical chain. 
A
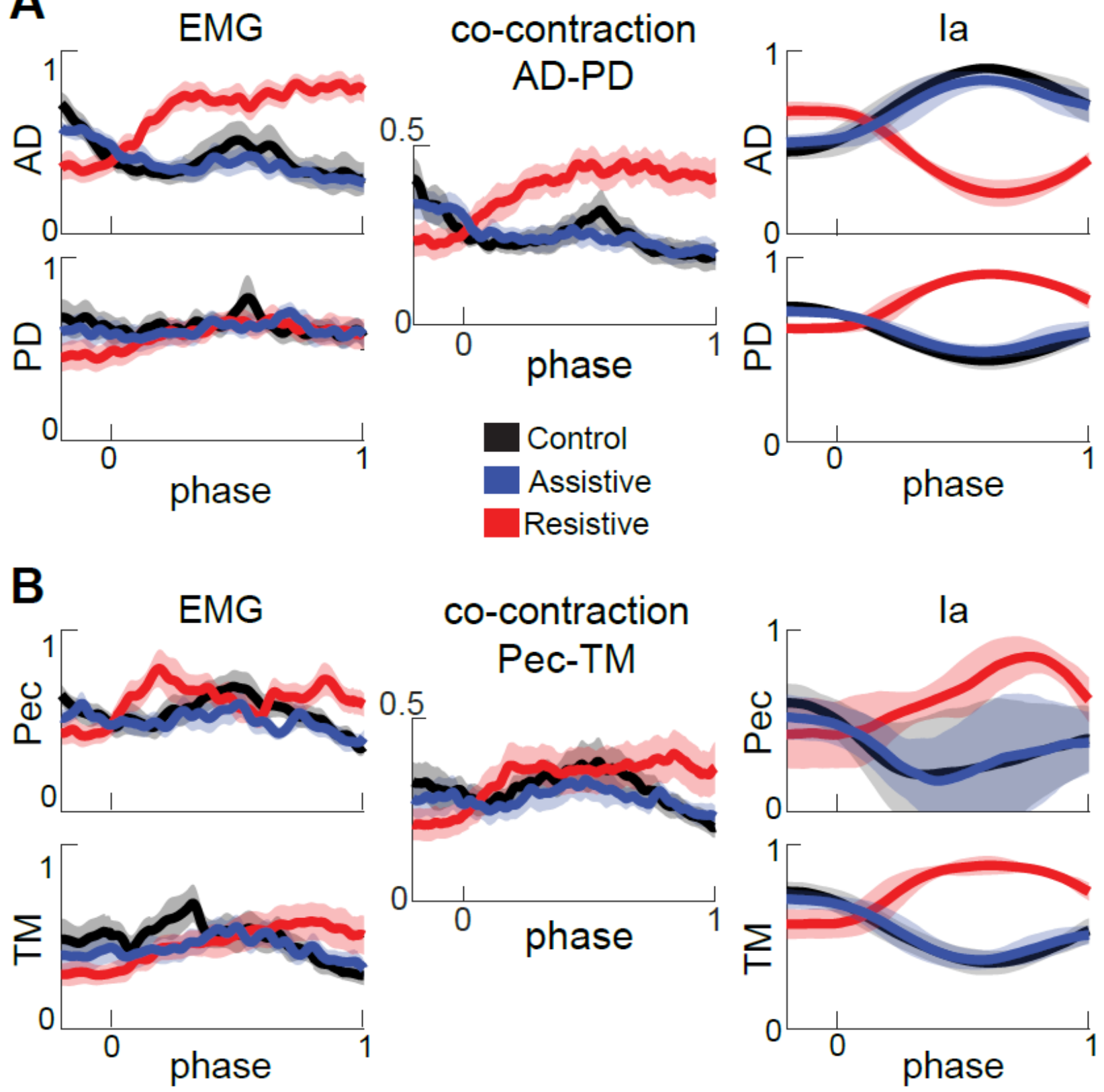

Figure 3-3: Normalized EMG, co-contraction, and la profiles for muscles spanning the shoulder.

Thick lines show averages for each movement across all participants. Shaded areas show the standard error of the mean across participants for EMG and co-contraction signals and standard deviation across participants for la signals. Movement phase represents normalized duration of each movement as in Fig. 3-2. Signals are subdivided into antagonist pairs AD-PD (A), Pec-TM (B), and BicL- TriLo (C). 

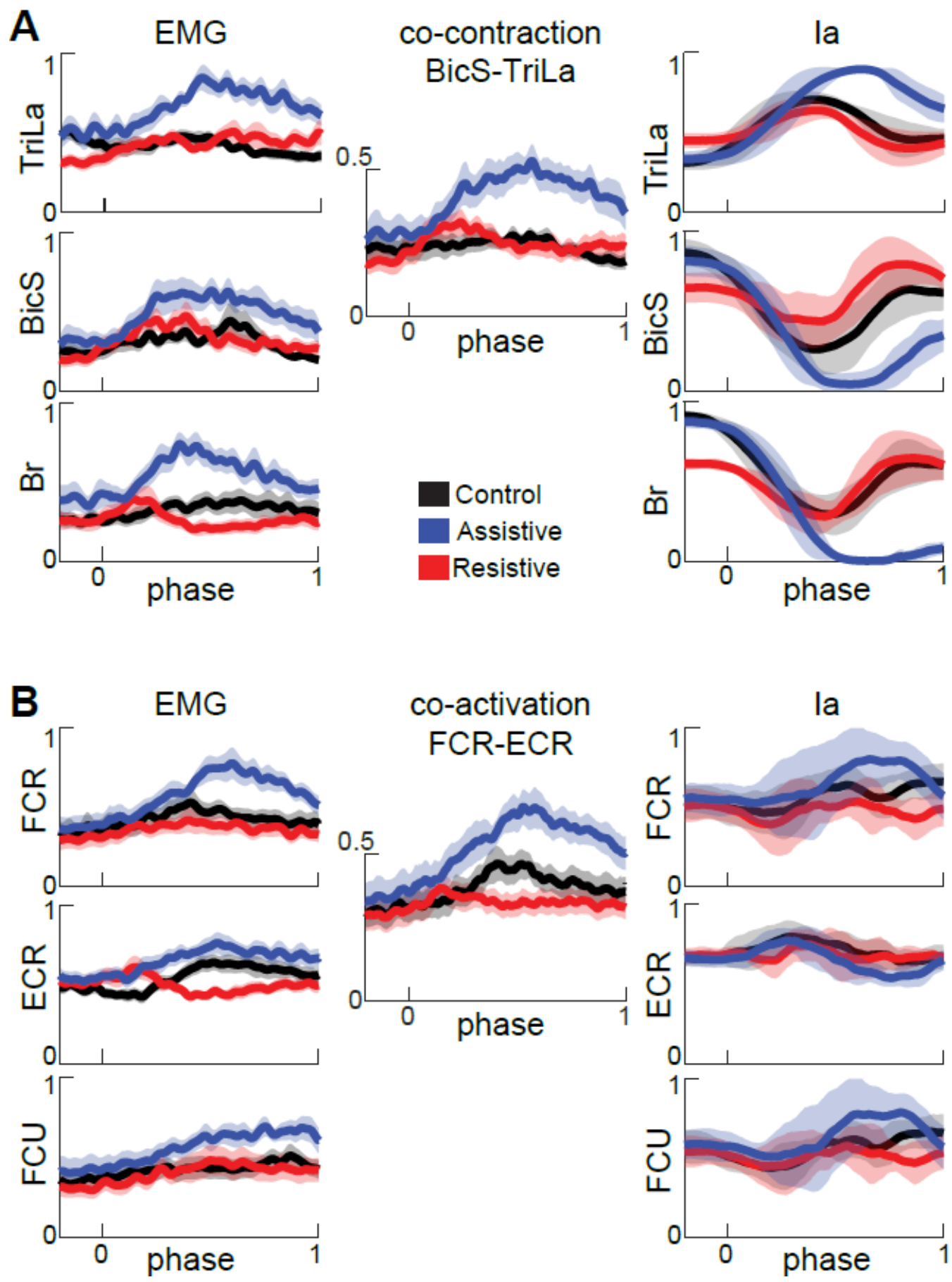

Figure 3-4: Normalized EMG, co-contraction, and la profiles for muscles spanning the elbow and wrist.

Formatting of plots are as in Fig. 3-3. Signals are subdivided into antagonist pairs Br, BicS-TriLa (A) and FCU, FCR-ECR (B). 
The EMG and la profiles varied between tasks, but in a different manner from each other (Fig. 3-3, 3-4). The EMG profiles showed variable levels of co-contraction that changed between tasks and joints. In the Assistive task, in which passive forces assist at the shoulder, the EMG profiles of multiple muscles that span the elbow and wrist joints were ramping up during movement (Fig. 3-3C \& 3-4, blue lines). The co-contraction between pairs of antagonists represented by shared variance between their normalized EMG profiles was the largest in the Assistive task (Supplementary Table S1). Hierarchal cluster analysis has shown that in the Assistive task multiple agonists and antagonists spanning the elbow and wrist (TriLa/TriLo/BicL/BicS/Br/FCR/FCU/ECR) comprised a single EMG cluster, i.e. all these muscles co-activated in this task (Fig. 3-5 Assistive). In the Resistive task with the least reliance on passive dynamics, co-contraction was high in AD-PD (Fig. 3-3A) and low in elbow and wrist muscles (Fig. 3-3C, 3-4; Supplementary Table S1). Hierarchal cluster analysis has shown that in the Resistive task, muscle coactivation was present in two different smaller clusters (Br/FCR/FCU/ECR and TM/AD/PD/TriLa; Fig. 3-5 Resistive), while in the Control task muscle coactivation was present in even smaller clusters (Br/FCR/FCU/ECR, TriLo/TriLa, BicS/BicL, and Pec/TM; Fig. 3-5 Control). Overall this analysis shows that the muscle groups defined by shared variance across EMG profiles are task-dependent and that they consist of both agonistic and antagonistic muscles. 
A

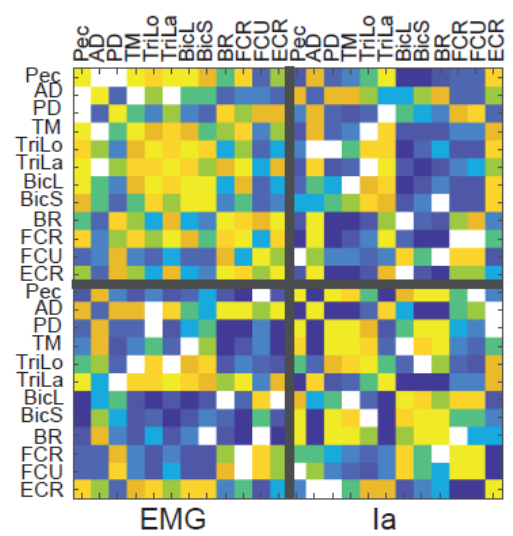

B

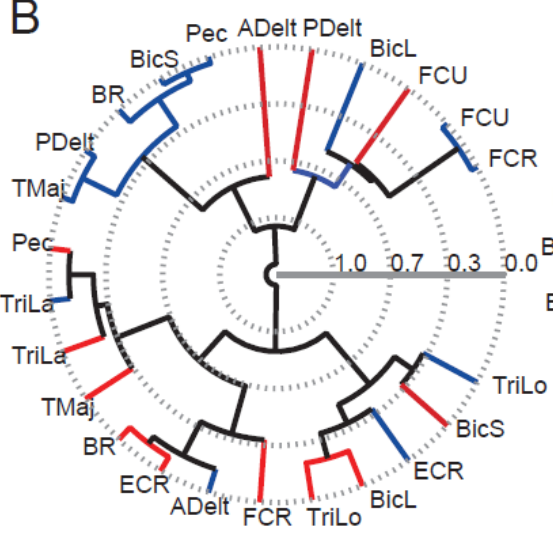

Resistive

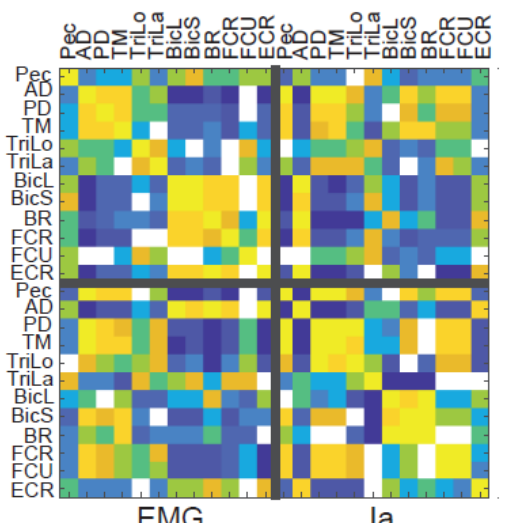

EMG

la

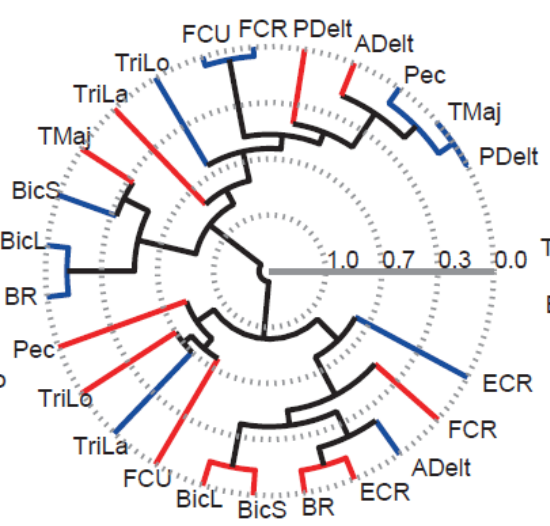

Assistive
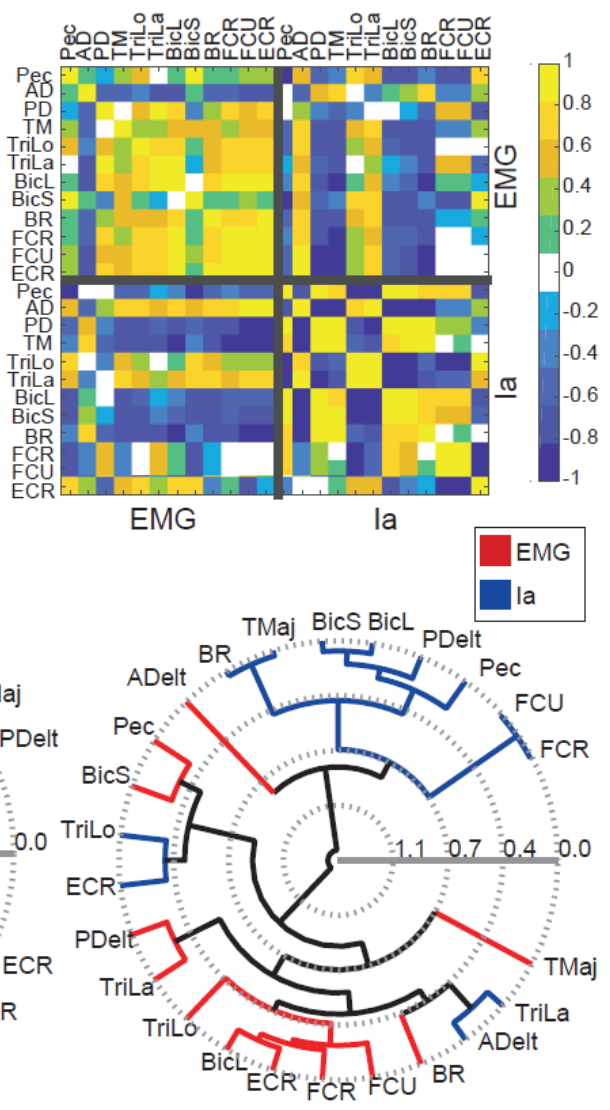

Figure 3-5: The relationships between EMG and la profiles per task in a representative individual.

A. The correlation matrix between normalized EMG and la profiles one participant. B. Hierarchical clustering of the correlation matrix in A. Lines represent the strength of the relationship between each cluster at different cluster subdivisions. 

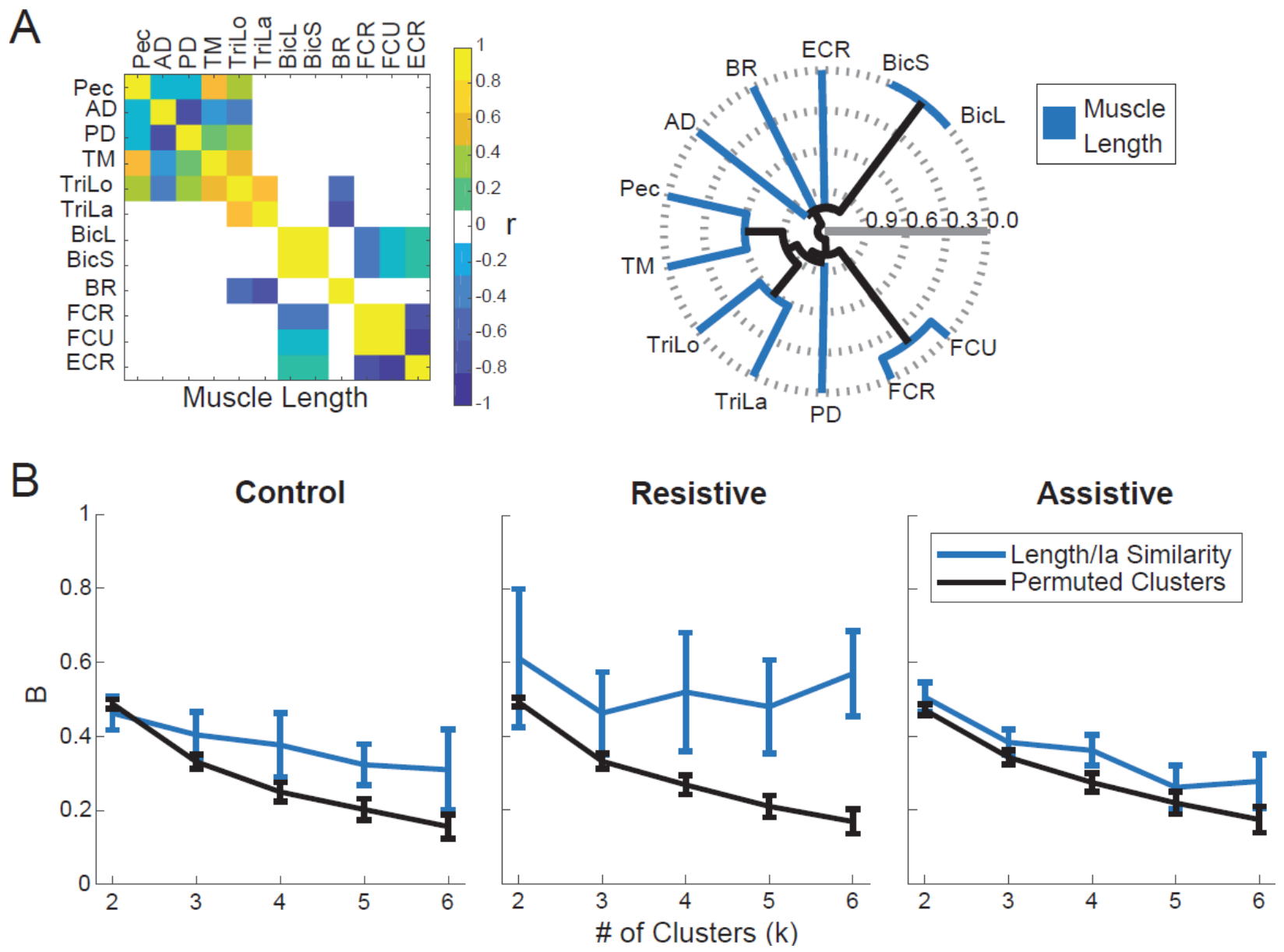

Figure 3-6: Comparison between muscle length and la clustering.

A. The relationships between muscle length profiles per task in a default "average subject" model used in (Gritsenko et al., 2016). The plots are formatted as in Fig. 3-4. B. Fowkles-Mallow Index (B) for the comparison between muscle length and la cluster assignments (blue) and for the comparison between muscle length and permuted la cluster assignments representing random match (black) at different cluster subdivisions. Error bars shows pooled standard deviation across participants.

In contrast to EMG profiles, the la profiles largely reflected the kinematic differences between tasks. For example, the la profiles of muscles spanning only the shoulder reversed in the Resistive task, in which the direction of shoulder excursion reversed relative to Control and Assistive tasks (Fig. 3-2, 3-3A \& B); the la profiles of muscles spanning the elbow largely followed the profiles of elbow excursions (Fig. 3-2, 3-4). The amount of shared variance between la profiles from antagonistic muscles did not change between tasks and the correlations were primarily negative, except for Pec-TM (Supplementary Table S2). The negative correlations between la profiles of antagonists 
are consistent with the reciprocal actions of antagonistic muscles. This also suggests that Pec and TM are not acting as antagonists in the selected movements. The la profiles of smaller groups of mainly agonistic muscles, such as Pec/PD/TM, TriLo/TriLa, $\mathrm{BicS} / \mathrm{BicL} / \mathrm{Br}$, and FCR/FCU, were positively correlated (Fig. 3-5A, right bottom corners of correlation matrices). Hierarchal clustering analysis comparing la profiles and muscle length from Gritsenko et al.(Gritsenko et al., 2016) showed that the la clusters were significantly more similar than expected by chance to muscle length clusters for all tasks at most cluster subdivisions (Fig. 3-6, Supplementary Table S3). Thus, we have supported the $1^{\text {st }}$ hypothesis as expected. The significant similarity index at multiple cluster subdivisions confirms that the same muscles that shorten or lengthen together also have similar la feedback across multiple postures or movements. Overall, this analysis suggests that the la afferents in synergistic muscles signal similar information related to the kinematics of reaching.
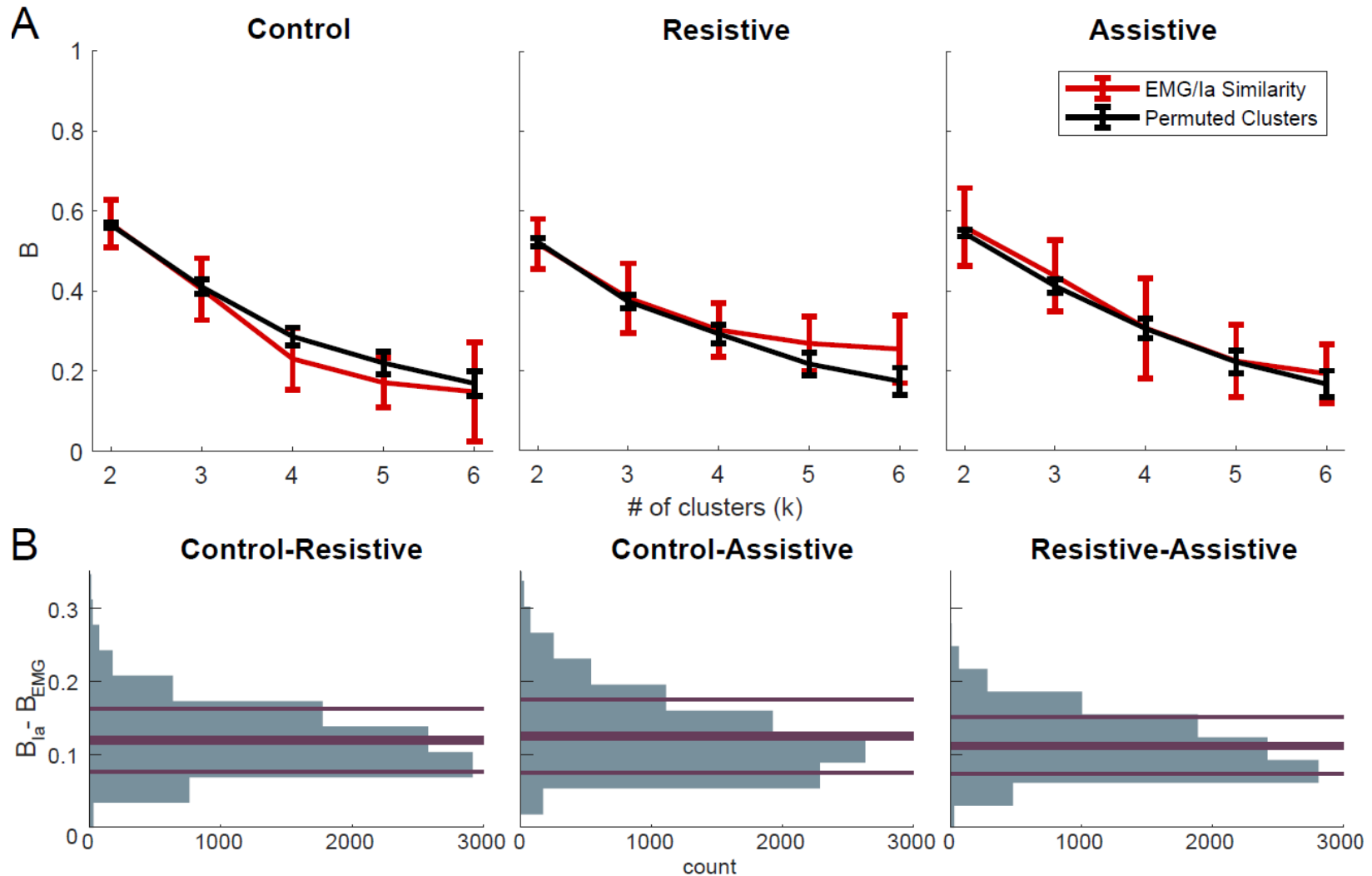

Figure 3-7: The consistency of hierarchical clustering between EMG and la within and across movements.

A. Fowkles-Mallow Index (B) for the comparison between EMG and la cluster assignments (red) and for the comparison between EMG and permuted la cluster assignments representing random match (black) at different cluster subdivisions. Error bars shows pooled standard deviation across participants. $\boldsymbol{B}$. 
Histograms of the differences in $B_{l a}$ and $B_{E M G}$ between tasks across bootstrapped hierarchal cluster trees. The abscissa indicates no difference in $B$ values between tasks; thick line is at the mean of the distribution; thin lines show standard deviations of the distributions.

To address the question of la afferent contribution to muscle co-contraction, we compared the time varying normalized la and EMG profiles in each task (Fig. 3-5A, right top corners of correlation matrices). The shared variance between la and EMG profiles from homonymous muscles was variable between muscles and tasks in both the strength and sign of the correlation (Supplementary Table S4). Pectoralis and biceps muscles shows the largest negative correlations, while the triceps muscle showed that largest positive correlations. This indicates that la feedback can both potentiate and inhibit the activity of its homonymous muscle in different tasks, even at the same level of static set across tasks represented by unchanging coefficients $A$ and $B$ from Eq. (1). The direct contribution of la afferents to muscle co-contraction can be quantified with hierarchal cluster analysis that groups positively correlated EMG profiles of co-contracting muscles and their la profiles into the same clusters. This predicts that, for example, the two clusters of co-activating muscles in the Resistive task should also contain the la profiles from the same muscles so that the Fowlkes-Mallows similarity index $(B)$ between EMG and la clusters would lie outside the noise distribution. However, the distribution of similarity indices between EMG and la clusters was indistinguishable from noise at most cluster subdivisions in all tasks (Fig. 3-7A, Supplementary Table S5). This result did not support the $2^{\text {nd }}$ hypothesis, indicating that the similarity between la and EMG clusters is spurious. Further analysis comparing directly the co-contraction profiles from antagonists to the la profiles from their host muscles further supported the lack of similarity in profiles. It showed that the la profiles from antagonistic muscle pairs were correlated reciprocally, either positively or negatively but never both positively, with the corresponding cocontraction profile (Supplementary Table S6). This indicates that muscle co-contraction can be potentiated by monosynaptic la feedback from one of the antagonistic muscles, but not both.

To contribute meaningfully to co-contraction, the la profiles from co-contracting muscles need to change between tasks the same way as EMG profiles of these muscles change between tasks. Therefore, we compared cluster structure between tasks. We 
observed that the similarity of la clusters $\left(B_{l a}\right)$ between tasks was higher than the similarity of EMG clusters $\left(B_{E M G}\right)$ between tasks (Fig. 3-7B; $p<0.001$ for all task comparisons). This shows that la clusters were more consistent between tasks than EMG clusters were (Fig. 3-6B). Therefore, $3^{\text {rd }}$ hypothesis that the EMG and la clusters change the same way between tasks was not supported. Instead, this result supports the conclusion above that la clusters contain information related to the kinematics of reaching, which changed less between our tasks than limb dynamics and co-contraction did.

Lastly, we evaluated to what extent the fusimotor drive could alter la signal profiles to capture muscle co-contraction. To achieve this, we manipulated la model coefficients to simulate alternative fusimotor inputs, such as static set and $\alpha-\gamma$ coactivation. The models of different static sets with large coefficients produced firing rates that were above those reported for human large fiber afferents (Human afferents from(Malik et al., 2016): $40 \mathrm{imp} / \mathrm{s}$; simulated afferents from Pec: $174 \pm 49 \mathrm{imp} / \mathrm{s}$; AD: $331 \pm 48 \mathrm{imp} / \mathrm{s}$; PD: $332 \pm$ $49 \mathrm{imp} / \mathrm{s}$; TM: $305 \pm 37 \mathrm{imp} / \mathrm{s}$; TriLo: $170 \pm 25 \mathrm{imp} / \mathrm{s}$; TriLa: $243 \pm 38 \mathrm{imp} / \mathrm{s}$; BicL: $171 \pm$ $49 \mathrm{imp} / \mathrm{s}$; BicS: $177 \pm 63 \mathrm{imp} / \mathrm{s}$; Br: $304 \pm 93 \mathrm{imp} / \mathrm{s}$; FCR: $129 \pm 42 \mathrm{imp} / \mathrm{s} ;$ FCU: $139 \pm 40$ $\mathrm{imp} / \mathrm{s}$; ECR: $142 \pm 35 \mathrm{imp} / \mathrm{s}$ with SD across participants). However, the maximal simulated firing rates increased linearly with the increases in model coefficients (data not shown). Therefore, the conclusions drawn based on the data simulated at extremes using models with large coefficients will apply to the data obtained using models with lower coefficients. 


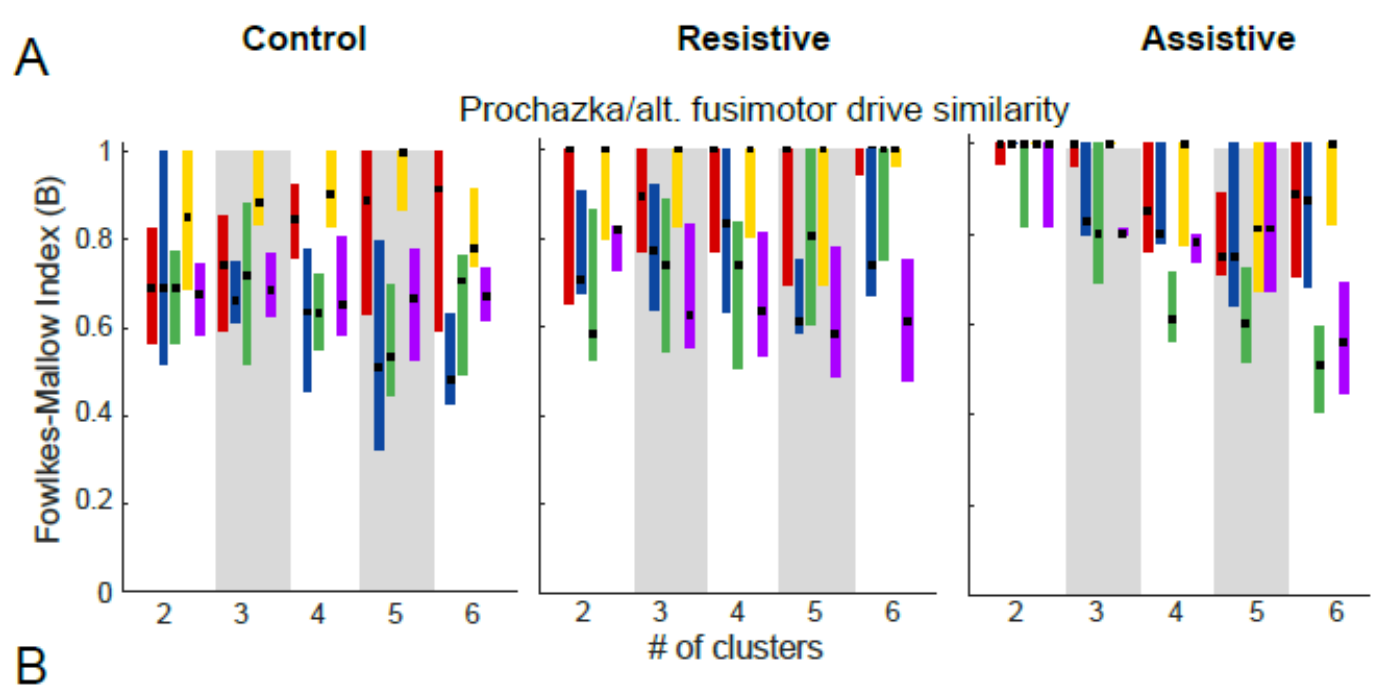

EMG/la similarity across fusimotor drive parameters
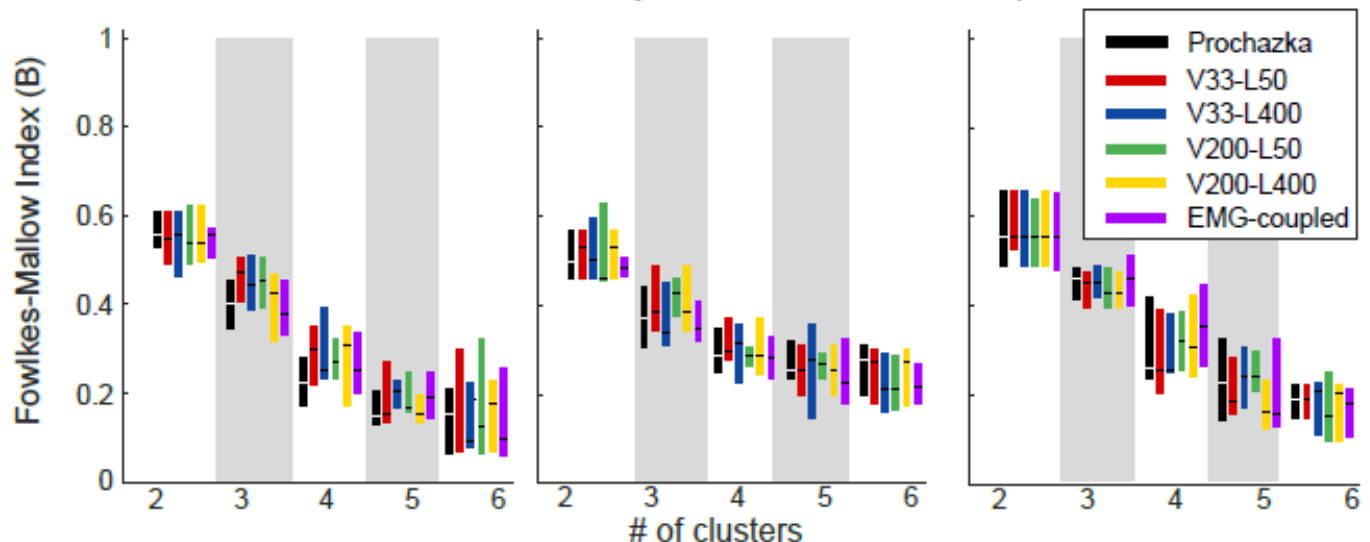

C

EMG/la similarity for Prochazka vs. alternate models
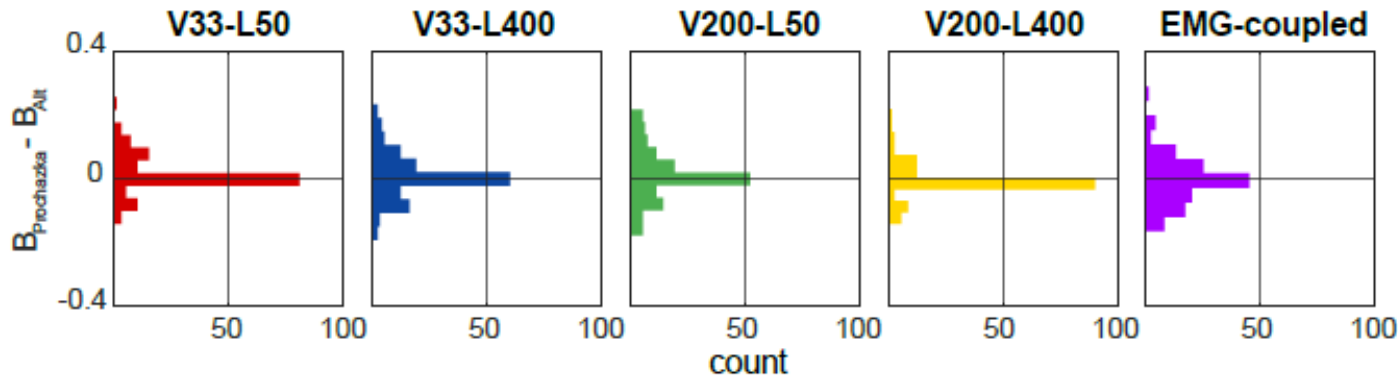

Figure 3-8: Fusimotor-based changes in the clustering of la profiles.

A. Similarity indices B between clusters produced by models with altered fusimotor coefficients and those produced by the original Prochazka model, boxes define the interquartile range across participants with the medians denoted by dots. B. Similarity indices B between EMG and la clusters for models with altered coefficients. Formatting is the same as in A. C. The histograms of differences between B of the Prochazka model and each of the alternative la models across individual participants, tasks, and cluster subdivisions. Zero difference is denoted by horizontal lines. 
We first evaluated how different static sets and $\alpha-\gamma$ coactivation can change the clustering structure of la profiles relative to that produced by the model with original coefficients (Prochazka model). We found that altering the la model coefficients did affect the clustering pattern of la signals as evident from the similarity indices between alternative models and the Prochazka model being less than 1 (Fig. 3-8A). The largest changes in la cluster structure were caused by the EMG-coupled la model simulating $\alpha$ $\gamma$ coactivation compared to those simulating altered static set. However, these changes in cluster structure did not increase the similarity between EMG and la profiles (Fig. 3$8 \mathrm{~B})$. Therefore, last set of hypotheses stating that the similarity between la and EMG clusters is not increased by alternative fusimotor drives were not supported (Fig. 3-8C; Supplementary Tables S7-S11). This shows that the changes in the la afferent activity caused by known fusimotor input, such as static set or $\alpha-\gamma$ coactivation, are not likely to potentiate sufficiently the amount of monosynaptic la feedback to the co-contraction of antagonists.

\section{Discussion}

Here we addressed the question of the degree to which the la afferent activity from muscle spindles in antagonists can contribute to their co-contraction through monosynaptic feedback under normal conditions, such as reaching movements. We asked human participants to reach toward virtual targets at different locations, which instructed planar movements in a transverse vertical plane. These reaching tasks were accompanied by different roles of passive limb dynamics, assistive or resistive. We found that EMG patterns changed between tasks and were associated with different levels of co-contraction, while the la patterns did not change between tasks and were primarily reciprocal between antagonists. Altering la model coefficients to simulate different types of fusimotor drive, such as static set and $\alpha-\gamma$ coactivation, did not change these conclusions. Although these results cannot rule out any given motor control theory, they do constrain the space of possible neural control solutions. Our results suggest a limited contribution of direct projections from the la afferents to muscle co-contraction, even with "simple" task-dependent changes in the fusimotor drive, such as static set and $\alpha-\gamma$ coactivation. 
The reaching tasks selected for this study represent unique dynamical contexts experienced by the multisegmented limb during movement in the presence of gravity. This was reflected in different active muscle torques and mechanical muscle work around the major joints in the three tasks (Table 1). Motion in the Control and Assistive tasks was produced with reliance on passive interaction torques and gravity. In the Assistive task, this was accompanied by the coactivation of the largest group of muscles (Fig. 3-3, 3-4). This may have served to increase distal limb stiffness, which helped to stabilize the movement against the potentially de-stabilizing whiplash interactions between joints(Hogan et al., 1987a; Burdet et al., 2001; Darainy and Ostry, 2008; Tee et al., 2010; Gritsenko et al., 2011b). In contrast, motion in the Resistive task was produced against the opposing action of gravity and interaction torques between shoulder and elbow. This was accomplished with concentric contractions of two different groups of proximal and distal muscles, biarticular biceps and triceps muscles changed their coactivation patterns the most (Fig. 3-3, 3-4). Overall, our results suggest that the dynamical demands of each task define specific patterns of coactivation of agonist and antagonist muscles that form broadly defined proximal and distal groups. These flexible task-dependent groups of coactivating muscles may reflect the neural compensation of limb dynamics through limb impedance(Hogan et al., 1987b; De Serres and Milner, 1991; Loeb et al., 1999; Burdet et al., 2001; Perreault et al., 2001; Damm and Mclntyre, 2008; Wong et al., 2009). ${ }^{38,55}$

There is a known monosynaptic relationship between la afferents and motoneurons innervating the same and synergistic muscles that underlies stretch reflexes, which compensate for perturbations. This anatomical arrangement with high gain, i.e. strong coupling, could result in similar profiles of the activity of la afferents and the profiles of the activity of homologous motoneurons, measured with EMG. Here, we tested this idea using two methods, hierarchal clustering of the correlation matrix between simulated la and EMG profiles and shared variance between profiles of antagonist co-contraction and la profiles. Hierarchal clustering revealed low similarity between la and EMG clusters, that resulted from inconsistent positive correlations between EMG and la profiles from the same muscles across tasks (Fig. 3-7; Supplementary Table S5). The comparison of cocontraction profiles with la profiles showed that only one of the antagonistic muscles was associated with positively correlated profiles, but not the other, and those relationships 
varied across tasks (Supplementary Table S6). Furthermore, we found that changing the parameters of the la model to simulate different levels of fusimotor static set between tasks did not increase the similarity between la and EMG clusters (Fig. 3-8). This suggests that simply changing the constant level of fusimotor drive between tasks cannot transform the la afferent activity so that it could contribute more to the co-contraction of antagonistic muscles observed during reaching movements. This may explain the findings of decreased gain of $\mathrm{H}$-reflexes, which are indicative of the strength of the monosynaptic connection between the la afferents and a motoneurons, during tasks that require more co-contraction, such as learning to co-contract antagonistic muscles during standing reduces the gain of soleus $\mathrm{H}$-reflex in humans(Perez et al., 2007). A recent simulation study has also shown that low gain of afferent feedback, both la and Ib, combined with co-contraction driven by mainly descending signals results in the optimal combination of stable control of movement and timely response to perturbations(Dideriksen et al., 2015). Altogether, this suggests that the monosynaptic la afferent feedback needs to be modulated nonlinearly during movement to contribute significantly to the co-contraction of antagonistic muscles.

Here we explored one type of nonlinear fusimotor drive that coupled the changes in muscle spindle sensitivity to muscle length and its rate of change to the activity of homonymous motoneurons. Such $\alpha-\gamma$ coactivation is thought to potentiate the recruitment of homonymous motor pools, increase muscle stiffness, and decrease the response times to perturbation(Pruszynski et al., 2009; Crevecoeur and Scott, 2014). Our results have shown that the nonlinear transformation of the la afferent signal by $\alpha-\gamma$ coactivation can change the profiles and, consequently, the clustering of la afferent signals more than all other models (Fig. 3-8A, EMG-coupled model). However, these changes were not enough to alter the la afferent profiles in a way that would reflect muscle co-contraction (Fig. 38B \& C). This suggests that another type of nonlinear transformation of la afferent signals that is independent of signals modulating the activity of $\alpha$ motoneurons is required for la afferent-based co-contraction of antagonistic muscles. This transformation could either be applied through a complex nonlinear profile of fusimotor drive or nonlinear modulation of the gain of la afferent feedback onto the a motoneurons through presynaptic inhibition and/or spinal interneurons. Our results and approach can be used to test the first 
possibility. We can derive the temporal profile of the static and dynamic $\gamma$ motoneuronal activity that would create the task-dependent coactivation pattern seen in EMG by solving Eq. 2 with least squares for two separate a coefficients (static and dynamic) for every phase of movement using EMG co-contraction as a cost function. The second possibility is more open-ended. The second type of nonlinear transformation may be accomplished by state-dependent nonlinear presynaptic inhibition of the monosynaptic pathway from la afferents to a motoneurons in some of the co-contracting antagonistic muscles(Seki et al., 2003; Perez et al., 2007) or by state-dependent nonlinear inhibition of la interneurons that mediate the reciprocal inhibition of antagonists (for review see(Burke, 1999)). The propriospinal system can also be engaged in task-dependent modulation of afferent feedback gains(Roberts et al., 2008). Ultimately, the task-dependency is thought to be determined by the higher-level neural circuits that modulate presynaptic inhibition, fusimotor drive, and the activity of spinal interneurons(Feldman, 1966; Latash, 2008; Roberts et al., 2008; Pruszynski et al., 2009; Crevecoeur and Scott, 2014). Future perturbation studies that alter the la feedback, for example with vibration, during reaching with different dynamical contexts is the next logical step to test the predictions from this computational study.

A potential limitation of the la afferent model used here is its simplicity. More complex models of la afferents take into account intrafusal muscle properties and may have somewhat different profiles of la afferent activity(Chen and Poppele, 1978; Mileusnic et al., 2006). Specifically, these models capture the transient bursts in afferent firing due to short range stiffness of the intrafusal muscle fiber. It is not known how these transient bursts are used by the nervous system, a recent paper suggested they may help sense changes in muscle force(Blum et al., 2017). However, the scientific consensus is that the muscle spindle is primarily a sensor of muscle length and its rate of change, so that all models capture these features in their predictions of la afferent discharge. Therefore, our conclusions from the simple model are likely to be generalizable to simulations with other more complex models.

Another limitation our la simulations is the assumption that the muscle rest lengths is a halfway length between min and max of all possible muscle lengths across the whole physiological range of motion simulated in Gritsenko et al.(Gritsenko et al., 2016). We 
observed that the distributions of muscle lengths across degrees of freedom in the Gritsenko et al.(Gritsenko et al., 2016) were often not normal. Therefore, the half-way estimate of rest length could bias it to be outside of the most common operational range. To mitigate this limitation and test the generality of our results, we re-ran all analyses using rest lengths calculated differently. The new rest lengths were calculated as median lengths using the distributions from Gritsenko et al.(Gritsenko et al., 2016). The results were the same (data no included), further supporting the generalizability of our results.

The linear correlative approach used here to compare the primary la and EMG signals does not take into account the non-linear aspects of the transformation between them through the motoneuron pool. However, Farina et al. (Farina et al., 2014) have shown that motoneuron pools, unlike individual motoneurons, display linearizing properties in transforming the common synaptic input into the neural drive to the muscle, i.e. EMG. This shifts the bulk of non-linearities in the transformation from the monosynaptic la feedback to EMG toward other synaptic inputs, such as spinal interneuronal and descending inputs discussed above. Moreover, the non-linearities in the transformation from the activity of a motoneuron pool to EMG are likely to be minimal in the low range of $\sim 5-10 \%$ of maximal voluntary contraction during reaching (Tagliabue et al., 2015; Aurbach et al., 2020) examined here. At that range, the rate coding of recruited motor units is likely to drive linearly the EMG amplitude and, thus, muscle force (De Luca and Hostage, 2010). Therefore, within the constraints of our experiment the non-linear transformation of the monosynaptic primary la inputs to the motoneuron pool into EMG is likely to capture a smaller component of the transformation than the linear one quantified here.

\section{Chapter 4-Corticospinal Excitability is Modulated During Dynamically-distinct Reaching Tasks}

(this chapter has been submitted to eNeuro and is currently undergoing revisions based on peer review)

\section{Abstract}

The modality of neural control signals and how they are generated appropriately for the task demands are a matter of active debate. We used single-pulse transcranial magnetic 
stimulation over the primary motor cortex to measure how the excitability of the corticospinal tract is modulated during reaching tasks in humans. We designed unconstrained reaching tasks in virtual reality to precisely manipulate the passive forces acting on the human arm. During these tasks, we probed the amplitude and gain of the corticospinal contribution to the compensation for these forces. We observed active corticospinal control of all recorded muscles, as evidenced by the presence of motor evoked potentials in all muscles during stimulation below the resting motor threshold. We further found that during movement the corticospinal excitability was modulated proportionally to the motoneuronal excitability. Furthermore, the coefficient of proportionality was also modulated during motion in some muscles distinctly in each dynamic task.

\section{Introduction}

\section{Movement and Limb Dynamics}

The central nervous system (CNS) is thought to imbed the physical laws of nature, which determine how forces exerted by the muscles and tendons cause motion of body segments in the presence of anatomical constraints, inertia of the body, and external forces such as gravity or contact forces (Nishikawa et al., 2007b; Prochazka and Yakovenko, 2007c; Ting et al., 2015b; Hardesty et al., 2020). However, the location of this imbedding and means by which these physical laws are represented remain unknown. It has been shown that the motor commands generated by the primary motor cortex include the compensation for active interaction moments during planar reaching movements in humans (Gritsenko et al., 2011c) and monkeys (Kurtzer et al., 2006; Pruszynski et al., 2011) which may then recruit appropriate muscle activations via the corticospinal tract. Motor evoked potentials (MEPs) elicited by transcranial magnetic stimulation (TMS) provide a quantification of the underlying excitability of both pre- and post-synaptic elements of the corticospinal tract (Terao et al., 1995; Rothwell, 1997; Di Lazzaro et al., 2004; Chen et al., 2008; Bestmann and Krakauer, 2015). Changes in MEP characteristics therefore reflect changes in the neural state. For example, MEP magnitudes have previously been shown to be decreased during movement preparation suggesting increased cortical inhibition prior to movement onset (Rothwell). Furthermore, 
MEP magnitudes display a linear relationship with muscle activity under isometric conditions (Darling et al., 2006); however, this relationship has not previously been verified during active movements with distinct limb dynamics. We applied single-pulse transcranial magnetic stimulation (TMS) to the human primary motor cortex to perturb the inputs into the neuromechanical dynamical system and observed the gains of the control signals. This approach is commonly used in control systems engineering; here we have applied it to study the control systems of the human CNS. Notably, single-pulse TMS is rarely applied during motion of the limb due to the increased variability of the motor evoked potentials (MEPs), the amplitude of which depends on the excitability of motor cortex neurons, spinal interneurons, and motor neurons at the time of stimulation (Kiers et al., 1993; Thickbroom et al., 1999). Here we designed the study to control for several sources of this variability in order to obtain reliable MEPs during movement, which allowed us to examine the modulation of MEPs during posture and movement under different dynamical conditions.

\section{Materials and Methods}

\section{Subjects}

The WVU Institutional Review Board approved all procedures in this study (Protocol \#1309092800). Potential participants with any musculoskeletal pathologies or injuries, prior history of seizures or fainting, or tinnitus were excluded. We obtained informed consent prior to the start of experiments. We recruited 10 healthy human participants $(6$ male, 4 female, $24.3 \pm 1.8$ years old, $76.3 \pm 14.5 \mathrm{~kg}$ ). All participants reported to be righthand dominant.

\section{Motion capture and tasks}

Concurrently with EMG, we recorded reaching movements with motion capture using the Impulse system (PhaseSpace). We placed nine LED markers on bony landmarks of the arm and trunk using the best practice guidelines (Robertson et al., 2013a). Marker coordinates were sampled at $480 \mathrm{~Hz}$ using Recap software (PhaseSpace) and were low-

pass filtered at a cutoff frequency of $10 \mathrm{~Hz}$. The mean residuals of marker triangulation \pm 
standard deviation across subjects were $5.7 \pm 0.45 \mathrm{~mm}$. Joint angles were calculated by defining local coordinate systems for the trunk, humerus, forearm, and hand using at least 3 markers per rigid body. During the experiment, arm postures and reaching goals were defined using color-coded spherical targets $8 \mathrm{~cm}$ in diameter in virtual reality (VR) environment created using Vizard software (WorldViz) and Oculus headset (Fig. 4-1A). To minimize the inter-subject variability in kinematics, the locations of all targets were calculated based on subject's segment lengths in order to obtain common shoulder and elbow joint angles for each task (Table 1; wrist angle was instructed to be kept at zero and hand pronated). The VR system displayed the targets relative to each subject's shoulder marker on the acromion (Fig. 4-1B). The targets defined a set of "designer" movements in the sagittal plane with diverse dynamical contexts, where the movement was either largely passive (Control task), or interaction torques were resistive with increasing gravitational load (Resistive task), or interaction torques were assistive with decreasing gravitational load (Assistive task). The dynamical contexts of these movements and their selection was driven by inverse simulations performed with a dynamical model of the arm (Fig. 4-1B). Movements from different starting points were simulated in the presence of gravity to determine tasks which would vary the assistive/resistive interaction torques. The movements that most varied the dynamical conditions while maintaining similar endpoint trajectory length were selected.

To perform the tasks, participants were asked to reach only with their right (dominant) arm (without moving the trunk) with their elbow close to their trunk and a neutral, pronated wrist. The three tasks were presented to the participants in the same pseudorandom order, i.e. a randomized task order was generated prior to the study's data collection and each participant received this same order. Each trial started with the appearance of start (green) and stop (red) targets (Fig. 4-1C). The colors and target locations did not change until the participants placed their index finger, indicated with a yellow sphere, into the start target. One second after this occurred, the stop target changed color from red to green, directing the participants to begin the movement (Fig. 4-1C). Each movement was repeated for 138 repetitions (total of 414 trials). During experiments, motion capture was used to visualize the participant's arm in VR. The marker locations were streamed to VR and used to represent the three main segments of the arm (hand, forearm, and upper 
arm) as a stick figure (Fig. 4-1A). Motion capture, electromyography (EMG), and virtual events were synchronized using custom hardware as described in Talkington et al. (Talkington et al., 2015).

A

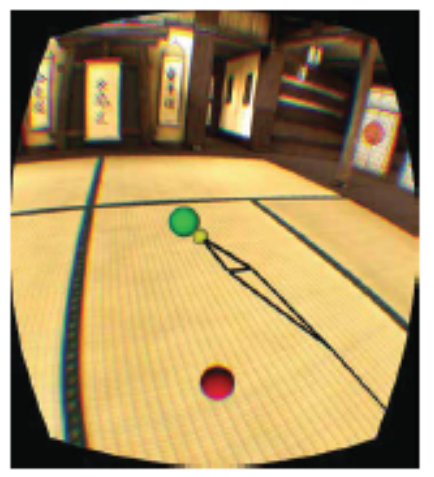

B

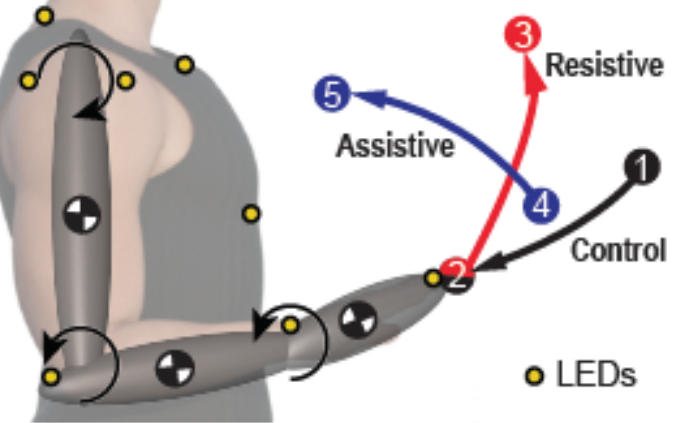

C

D

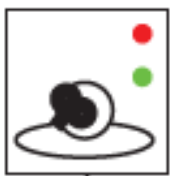

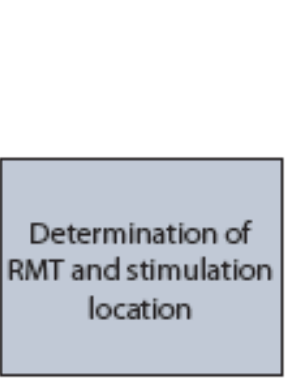
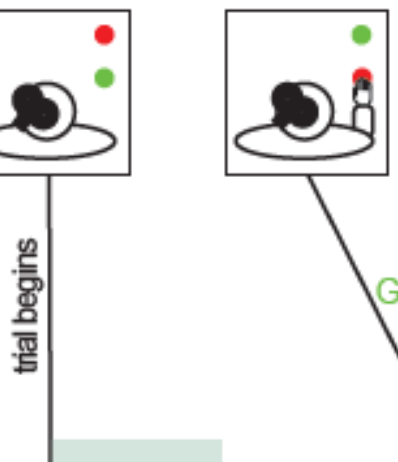

RT

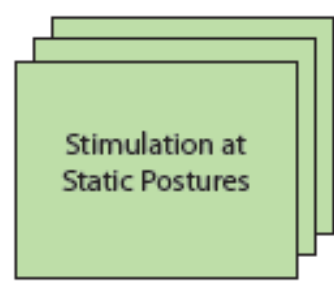

Experiment 1
90\% RMT

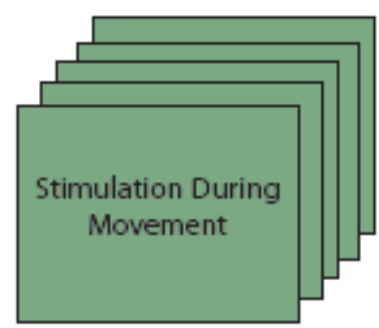

Experiment 2
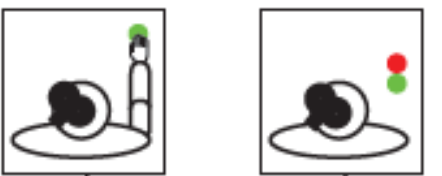

点

TMS

at pseudorandom

delay 
index finger, which the participants were instructed to move to the center of the green target. $B$. Schematic of the dynamic model used to calculate joint angles and torques from motion capture and representative endpoint trajectories from the three tasks. Positive directions for each joint are shown with black arrows. The start and stop target locations are shown for the Control, Resistive, and Assistive tasks (black, red, and blue, respectively). The motion capture marker locations are shown as yellow circles (marker on medial portion of wrist not visible). C. Visual representation of the experimental paradigm. D. Example timeline of events during a single trial of a movement task. RT stands for reaction time; GO represents the color cue to start movement.

Table 1: Posture defined by VR targets at start and end of each movement

Control Resistive Assistive

\begin{tabular}{|c|c|c|c|c|c|c|}
\hline \multirow[b]{2}{*}{ Shoulder } & $\begin{array}{l}\text { Target-defined } \\
\text { (deg) }\end{array}$ & initial & angle & -70 & -10 & -55 \\
\hline & $\begin{array}{l}\text { Target-defined } \\
\text { (deg) }\end{array}$ & final & angle & -10 & -55 & -10 \\
\hline \multirow{2}{*}{ Elbow } & $\begin{array}{l}\text { Target-defined } \\
\text { (deg) }\end{array}$ & initial & angle & 20 & 80 & 35 \\
\hline & $\begin{array}{l}\text { Target-defined } \\
\text { (deg) }\end{array}$ & final & angle & 80 & 90 & 135 \\
\hline
\end{tabular}

\section{Dynamics}

After experiments, motion capture was used to calculate active muscle torques at shoulder, elbow, and wrist joints using the same dynamical model used for the selection of tasks (Fig. 4-1B) as described in Olesh et al. (Olesh et al., 2017c). The model was customized to each participant's morphology by scaling model segment lengths to match the participant's arm length and scaling model mass to participant's weight using Winter's morphology (Winter, 2009b). We then ran inverse simulations using the motion capture data to obtain active torques produced by the muscles to make the movements in the presence of gravity and limb inertia. These active torques were then provided as input into a forward dynamics model and the simulated motion was compared to angular 
kinematics by calculating a root-mean-squared error (RMSE: $0.059 \pm 0.035 \mathrm{rad}$ ). Positive rotations, based upon the model's local coordinates are illustrated in Fig. 4-1B. Analysis was done on the angles and torques around the $X$ axes, i.e. flexion/extension degrees of freedom at all three joints, because motion was primarily in the sagittal plane and out-ofplane torques were negligible. The active muscle torques at each joint were then subdivided into gravitational and dynamic components to examine the different roles CNS may play in the compensation for gravity and movement production as described in (Olesh et al., 2017c).

\section{Electromyography}

Muscle activity and responses to TMS were recorded in twelve upper limb muscles using Trigno (Delsys Inc.), a wireless surface electromyography (EMG) system. The recorded muscles included four muscles spanning the shoulder, three muscles spanning both shoulder and elbow, two muscles spanning only the elbow, and three muscles spanning the wrist (Table 2). Muscles were identified based on anatomical landmarks and palpation during contraction; EMG sensors were placed on muscle bellies oriented longitudinally along the muscle fibers EMG signals were sampled at $2 \mathrm{kHz}$ with a gain of 1000 . EMG recordings were high-pass filtered at $10 \mathrm{~Hz}$ to remove any signal drift and rectified prior to any EMG/MEP quantification. EMG profiles from trials without TMS were low-pass filtered at a cutoff frequency of $20 \mathrm{~Hz}$ and normalized per subject using a maximum value for each muscle across all tasks.

Table 2: Muscles Recorded with EMG and Abbreviations

\begin{tabular}{lll}
\hline Muscle & Abbr. & Joints Spanned \\
\hline pectoralis & Pec & Shoulder \\
anterior deltoid & AD & Shoulder \\
posterior deltoid & PD & Shoulder \\
teres major & TM & Shoulder \\
triceps (long head) & TriLo & Shoulder, Elbow \\
triceps (lateral head) & TriLa & Elbow
\end{tabular}




\begin{tabular}{|c|c|c|}
\hline biceps (long head) & BicL & Shoulder, Elbow \\
\hline biceps (short head) & BicS & Shoulder, Elbow \\
\hline brachioradialis & BR & Elbow \\
\hline flexor carpi radialis & FCR & Wrist \\
\hline flexor carpi ulnaris & $\mathrm{FCU}$ & Wrist \\
\hline $\begin{array}{l}\text { extensor } \quad \text { carpi } \\
\text { radialis }\end{array}$ & ECR & Wrist \\
\hline
\end{tabular}

\section{Transcranial magnetic stimulation}

We assessed corticospinal excitability for each participant using single-pulse TMS delivered by the Super Rapid stimulator with a figure-of-eight coil (Magstim). The coil was placed tangentially to the scalp, oriented at a $45^{\circ}$ angle to the midline with the handle pointing posteriorly and laterally (Fig. 4-1C). The coil location over the scalp and its orientation was maintained using the Brainsight neuronavigation system (Rogue Research). The location of stimulation was selected using the hot-spot method (Traversa et al., 1997; Ellaway et al., 1998), during which the coil was moved over the estimated location of the primary motor cortex until a location with at least $50 \mu \mathrm{V}$ motor evoked potential (MEP) in BicS was evoked. This controlled for the anatomical differences between subjects and defined a consistent stimulation location on the motor homunculus (Penfield and Rasmussen, 1950b). This was done with anticipation that stimulating the same anatomical location with the lowest corticospinal excitability for BicS would produce proportionally similar responses in other muscles across participants. Resting motor threshold was then determined at the hot-spot location by varying the stimulation intensity until a MEP $>50 \mu \mathrm{V}$ was evoked $50 \%$ of the time in BicS. This procedure ensured that the stimulation amplitude was adjusted to individual differences in corticospinal excitability at rest at the time of experiment and, thus, further minimized intersubject differences in MEP amplitudes. TMS pulses were applied at intervals greater than $5 \mathrm{~s}(0.2 \mathrm{~Hz})$ to avoid any long-term changes in corticospinal excitability (Chen et al., 1997). The experiment consisted of two consecutive sessions conducted on the same day. 


\section{Session 1: static posture}

To evaluate the "background" state of the motor system for maintaining posture without goal-directed motion, we measured MEPs when the participants held their arm at postures corresponding to locations of virtual targets used to direct motion tasks (Fig. 41B). Participants held their arm in one of five static postures, as the starting position for the Resistive task was the same as the ending position for the Control task. Participants were asked to reach to the displayed VR target that corresponded to one of the postures and hold their arm in that posture while we applied 12 TMS pulses at $<0.2 \mathrm{~Hz}$ at $90 \%$ of resting motor threshold. They were then instructed to reach to the next target and hold the next posture, etc.

\section{Session 2: movement trials}

Movement trials during three tasks described above were used to probe corticospinal excitability at multiple time points directly preceding and during movement. These trials were divided into stimulation trials (126 per movement, 378 total) with non-stimulation control trials (12 per movement, 36 total) interspersed randomly. TMS was performed at $90 \%$ of the resting motor threshold. In one half of trials (189 of 378), the TMS was triggered at a random delay of $0-550 \mathrm{~ms}$ after the participant touched the start target. This triggering method targeted times directly preceding or directly after movement onset. In the other half of trials, the TMS pulses were triggered when the participant left the start target after a random delay of $0-550 \mathrm{~ms}$. These trials targeted TMS towards movement offset. The different triggering events ensured a distribution of TMS pulses prior to and during movement. The timing of each TMS pulse was recorded relative to both EMG and kinematics for post-hoc synchronization, binning, and analysis.

\section{Session 1 MEP analysis}

We quantified the probability of evoking MEPs in the biceps muscle by comparing the peak-to-peak amplitude of MEPs to the variation of EMG amplitude preceding stimulation. The maximum peak-to-peak amplitude was calculated using a $40 \mathrm{~ms}$ window of time either directly following stimulation (MEP) or $5 \mathrm{~ms}$ preceding stimulation (EMG) for single trials. We defined the presence of a MEP as a peak-to-peak amplitude of at least 5 standard 
deviations above EMG amplitude. The probability was calculated by dividing the number of detected MEPs by the number of stimuli under the same conditions. MEP latencies were calculated using a procedure similar to that used to determine kinematic onsets. EMG recordings were low pass filtered at $100 \mathrm{~Hz}$ and averaged across trials. A local maximum was found for the third derivative of this averaged signal.

We assessed postural corticospinal excitability by quantifying MEPs in static postures. Rectified EMG was first integrated over a $40-\mathrm{ms}$ period beginning $10 \mathrm{~ms}$ after each TMS pulse. The selected window ensured that the integrated time period encompassed the entire MEP. MEP amplitude (MEP amp $)$ during isometric contraction has been shown to follow the Boltzmann equation (Darling, 2006; Devanne, 2002):

$$
M E P_{a m p}=\frac{M E P_{\max }}{1+e^{\left(\frac{S_{50}-S}{k}\right)}}+B \times \operatorname{PreEMG}+C_{1}
$$

Where $M E P_{\text {max }}$ is the maximum MEP amplitude, $\mathrm{S}_{50}$ is the stimulation intensity to elicit an MEP at $50 \%$ of the maximum amplitude, $S$ is the stimulation intensity, and PreEMG is the amplitide of EMG measured directly preceding stimulation, and $C_{1}$ is a constant. In this study, we performed all stimulations at a consistent intensity of $90 \%$ the RMT, which makes the first term a constant. Therefore equation (1) simplifies to the following:

$$
M E P_{a m p}=B \times \operatorname{PreEMG}+C_{2}
$$

The PreEMG term was calculated by integrating rectified EMG over a $40-\mathrm{ms}$ period prior to TMS pulse. The slope $B$ was used to normalize the MEP amplitudes during movement as described below.

To determine the consistency of MEP amplitudes, we calculated the coefficient of variation (CV) across repetitions recorded per subject/muscle/time bin. 
Session 2 MEP analysis

The MEP magnitudes during movement are also dependent on changes in the motoneuronal excitability that are reflected in the profile of EMG in each task. We used the linear MEP-EMG relationship observed in static postures to normalize MEPs observed during movement to EMG. To normalize MEPs during movement we calculated predicted MEPs using the slopes or ratios between static MEPs and background EMG obtained from Session 1 as follows:

$M E P_{\text {Pred }}=B \cdot E M G_{\text {dynamic }}$

where $M E P_{\text {Pred }}$ is expected or 'predicted' MEP amplitude at a given phase of movement; $E M G_{\text {dynamic }}$ is the background EMG averaged across control trials without TMS in the corresponding phase, muscle, and task. These predicted MEPs were then used to normalize the MEPs obtained during movement at the corresponding phase. TMS responses during movement were grouped into 5 bins, each corresponded to $20 \%$ increments of phase duration from onset to offset of movement (Fig. 4-2). The $E M G_{\text {dynamic }}$ was similarly binned. To accurately estimate MEP amplitudes preceding movement, TMS responses that occurred up to $20 \%$ phase duration prior to movement were grouped into a bin 0 . This binning procedure amalgamated MEPs occurring at similar times during movement, providing adequate repetitions to estimate mean values. We defined a minimum repetition criterion of 5 MEPs based upon (Lewis et al., 2014). Bins that contained $<5$ MEPs were excluded from subsequent analyses.

Two measures were used for estimating corticospinal excitability. The first measure was MEP magnitude, which was defined as the ratio between the median MEP in a given phase during movement and the median MEP in the starting posture for the corresponding task and muscle. Thus, MEP magnitudes equal to 1 indicated that the MEPs during movement were equal to those at the starting posture for that movement. The second measure, Gain, was defined by equation 3:

$$
\text { Gain }=\frac{M E P_{D y n}}{M E P_{\text {Pred }}}
$$


where $M E P_{D y n}$ is the MEP magnitude during movement and the $M E P_{\text {Pred }}$ is calculated using eq. (3). The MEP gain equal to 1 means that the median MEP at a given phase is equal to the predicted MEP based on the linear relationship between EMG and MEPs described in eq. (2) with the same slope as that observed in static trials. MEP gain values $<1$ indicate a decrease in corticospinal excitability relative to that observed in static trials. By controlling for the linear relationship between EMG and MEP, the gain metric reveals changes in the corticospinal tract that are independent of motoneuronal excitability.

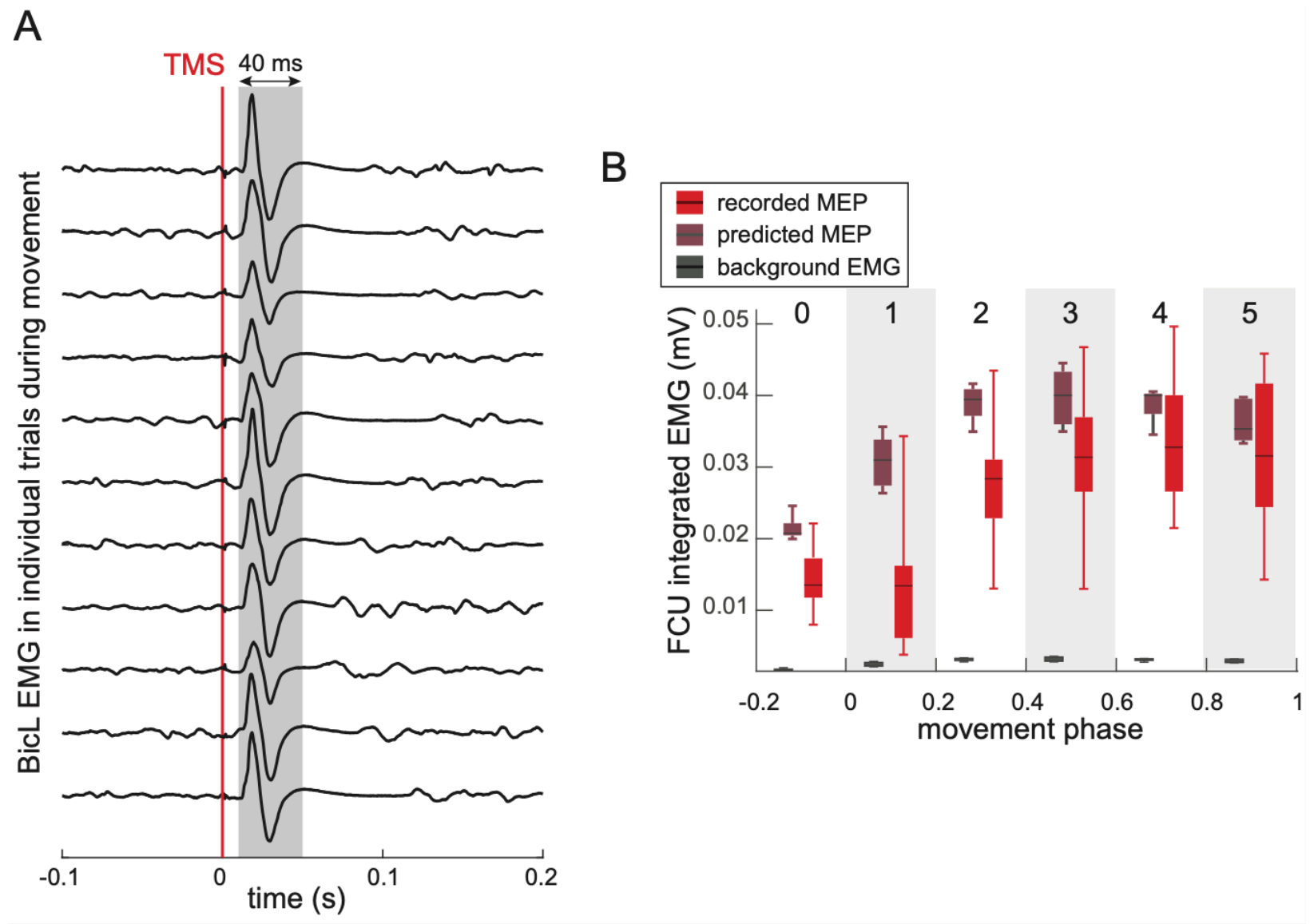

Figure 4-2: Quantifying TMS responses during movement

A. EMG in individual trials aligned on TMS pulse (red line). Grey area shows the integration window used to quantify MEP amplitude from rectified signals (not shown). B. Magnitudes of background EMG, predicted MEPs, and recorded MEPs binned according to the phase of movement, where 0 denotes the kinematic onset of movement and 1 denotes the kinematic offset. 


\section{Statistical Analyses}

The statistical analysis of corticospinal modulation relative to static condition was based on non-parametric bootstrap method because MEP amplitudes and gains were not normally distributed (Efron and Tibshirani, 1993). The MEP amplitude and gain values were bootstrapped independently for 100 iterations while preserving the structure of the data (Efron and Tibshirani, 1993). This means that single-trial MEPs and corresponding EMG values were sampled with replacement separately for each movement phase, each task, each muscle, and each participant. Each bootstrap was averaged first within each subject and then across subjects to maintain equal contribution of individuals to the overall distribution. Significance was determined using a percentile measure with alpha = 0.0001 with correction for multiple comparison using a conservative Bonferroni method. Significant values are reported in Figures 6 and 7 as dots above bars.

To test a hypothesis that the TMS gain profiles are linearly related to EMG and muscle torques, we performed two types of regression analyses. First, the amount of shared variance between binned EMG and TMS amplitude or TMS gain was calculated using the coefficient of determination $\left(\mathrm{R}^{2}\right)$ across all movement phases and tasks per muscle per participant. The amount of shared variance between binned joint torques and TMS amplitude or TMS gain was calculated using the coefficient of determination $\left(R^{2}\right)$ across all movement phases per task, muscle, and participant. The degrees of freedom were matched to muscles as follows, shoulder flexion/extension torques were compared to Pec, Ad, PD, and TM, elbow flexion/extension torques were compared to TriLo, TriLa, $\mathrm{BicS}, \mathrm{BicL}, \mathrm{BR}$, and wrist flexion/extension torques were compared to FCR, FCU, and ECR. Second, we created a generalized linear model to test whether MEP variance may be explained by a combination of EMG, kinematics, and torques. We used a stepwise regression to add or remove predictors based upon the Bayesian Information Criterion (BIC). All values in Results are means \pm standard deviations (SD) unless otherwise specified. 


\section{Results}

Corticospinal Excitability During Posture Maintenance

Maintaining limb posture against gravity requires active neural control to compensate for the complex anatomy of our bodies and the imprecise and noisy biological control signal (Harris and Wolpert, 1998). In the current study, we found evidence supporting the contribution of the corticospinal tract to this posture maintenance. At stimulation amplitudes below resting motor threshold, we observed MEPs in most muscles, even in those muscles that did not show changes in EMG associated with holding the arm against gravity. Specifically, the biceps long muscle participates in posture maintenance by holding the elbow and shoulder flexed in the tested postures (Fig, 4-3A). We found that the probability of evoking a MEP in this muscle was higher than expected from stimulation at the resting motor threshold in a majority of participants (Fig. 4-3B). Furthermore, the probability of evoking a MEP was higher than expected in all muscles, even those not directly involved in holding the arm against gravity (Pec: $0.81 \pm 0.14$; $A D: 0.77 \pm 0.23$; PD: $0.87 \pm 0.18$; TM: $0.88 \pm 0.15$; TriLo: $0.88 \pm 0.17$; TriLa: $0.92 \pm 0.12$; BicL: $0.93 \pm 0.14$; BicS: $0.90 \pm 0.17$; Br: $0.95 \pm 0.17$; FCR: $0.93 \pm 0.18$; FCU: $0.94 \pm 0.16$; and ECU: $0.95 \pm$ $0.17)$. 

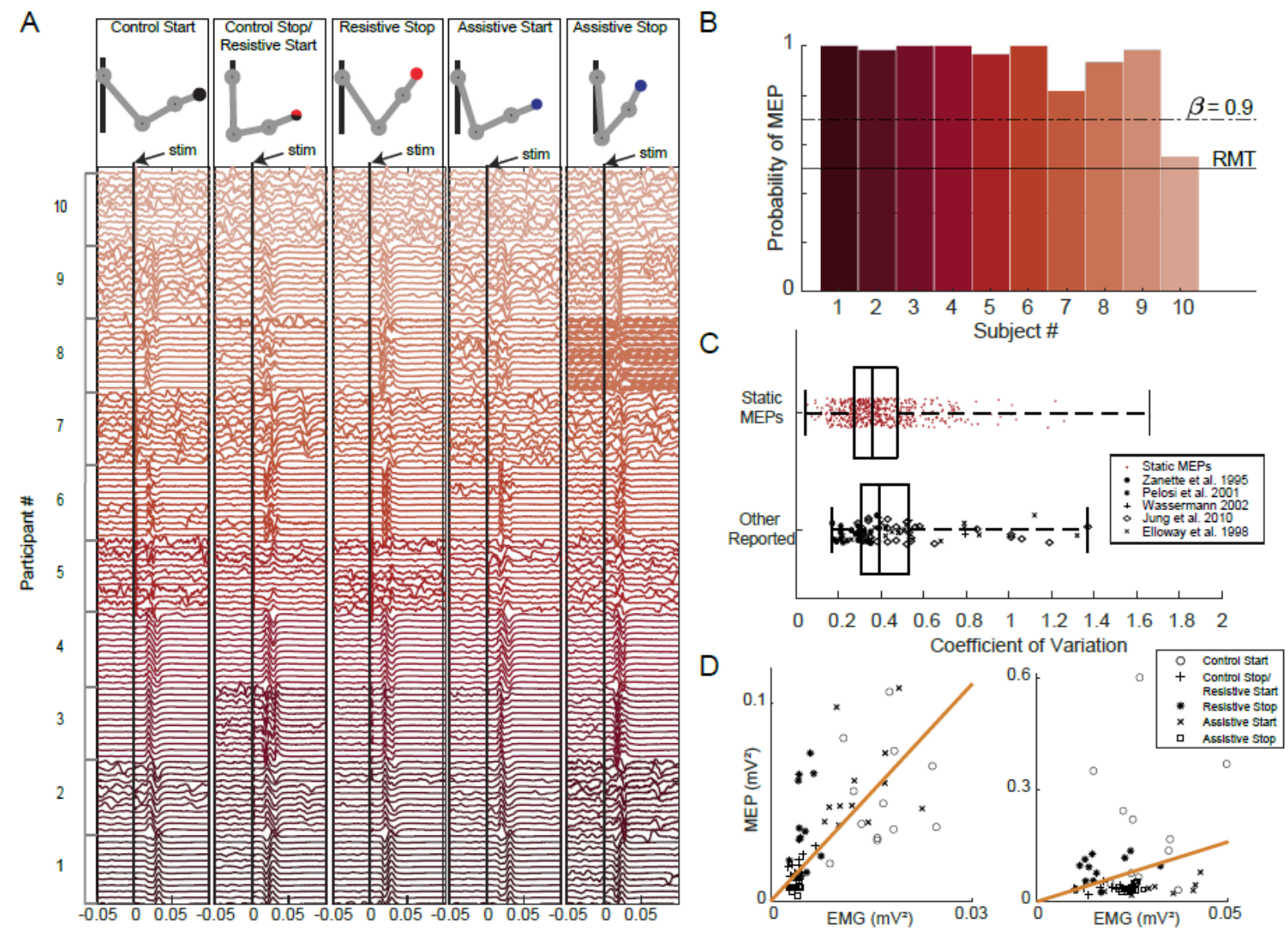

Figure 4-3: Postural corticospinal excitability in BicL

A. EMG traces of the biceps muscle during static postures are displayed for all participants. The black line corresponds to the time of TMS pulse (stim). B. Each bar corresponds to the probability of an MEP occurring for each participant across all postures. The solid black line denotes the probability of MEP occurrence at $R M T$ and the dashed line corresponds to the increase in probability that could be detected given the statistical power of our study design $(\beta=0.9)$. C. Coefficient of variation for all muscles in each posture are shown in red. The values from published studies are shown in black. $D$. The linear relationship between integrated MEP magnitude and background EMG. An example of the "best" (left) and "worst" (right) linear fit as determined by $R^{2}$.

MEPs observed during static postures displayed highly consistent characteristics with previous studies. MEP latencies $(15 \pm 1.9 \mathrm{~ms})$ were consistent with previously reported values for muscles of the upper arm indicating that the same motor cortical location was stimulated in each participant. The latencies broadly reflected the proximal to distal distribution of muscles (Pec: $15 \pm 3.2$; AD: $13 \pm 2.4$; PD: $13 \pm 3.0$; TM: $14 \pm 2.1$; TriLo: 13 \pm 2.9 ; TriLat: $12 \pm 1.1$; BicL: $13 \pm 1.1$; BicS: $14 \pm 3.2$; Br: $16 \pm 2.0$; FCR: $17 \pm 1.5$; FCU: $18 \pm 2.2$; ECR: $16 \pm 2.3 \mathrm{~ms}$ ). To determine the consistency of integrated MEP magnitudes, 
we calculated the coefficient of variation for MEP repetitions recorded in the same condition (Fig. $3 \mathrm{C}$ ). The median coefficient of variation across all muscles was 0.35 which is comparable to reported values recorded at rest.

MEP magnitudes during static postures were linearly related to the background muscle activity at the time of stimulation as predicted by the Botlzmann equation (Eq. 2). The linear model accounted for $25 \%$ of the MEP variance on average across muscles $\left(R^{2}=\right.$ $0.25 \pm 0.13$ ) with a small mean squared error (MSE $\left.=2.2 e^{-5} \pm 4 e^{-5}\right)$. The regression slopes were consistent across multiple muscles and subjects, the values of the slope $B$ from eq. (2) across subjects were Pec: 1.00 - 1.35 - 1.53 (lower - median - upper quartile ranges), AD: 0.93 - 1.76 - 3.13; PD: 1.15 - 1.97 - 3.64; TM: 1.13 - 1.66 - 2.09; TriLo: 1.36 - 2.31 4.22; TriLa: 1.47 - 3.09 - 5.46; BicLo: 1.56 - 4.41 - 6.83; BicS: 1.64 - 3.75 - 5.90; Br: 3.94 - 5.89 - 10.10; FCR: 1.73 - 4.85 - 7.44; FCU: 3.43 - 4.49 - 9.46; ECR: 2.84 - 5.05 - 5.58. We used the regressions from the linear model to control for the expected modulation with EMG in the analysis of MEPs during movement.

\section{The Role of Limb Dynamics During Reaching}

The locations of virtual targets used in this study were selected using a biomechanical model of the arm so that movement was accompanied by gravitational and other inertial forces in specific directions. These forces created unique challenges for the control of limb dynamics by the CNS and defined our Control, Assistive, and Resistive tasks (see Methods: Movement Tasks). The virtual targets were very effective in evoking the desired behavior and standardizing the movement kinematics and dynamics across all participants (Fig. 4-4). The shoulder motion was mostly passive in the Assistive task, which was associated with reducing gravitational active torque (Fig. 4-4C Shoulder blue) and the lowest dynamic muscle torque (Fig. 4-4D Shoulder blue). In contrast, the shoulder motion was produced actively in the Resistive task, which required increasing gravitational muscle torque (Fig. 4-4C Shoulder red) and large dynamic muscle torque (Fig. 4-4D Shoulder red). The same tasks created different dynamic conditions at the elbow and wrist. The Assistive and Resistive tasks were similar and associated with

decreasing gravitational muscle torque (Fig. 4-4C, Elbow \& Wrist, blue and red). The Resistive task was associated with the largest acceleration dynamic muscle torque at the 
elbow (Fig. 4-4D Elbow red), while the Assistive task was associated with the largest deceleration dynamic muscle torque at both the elbow and wrist (Fig. 4-4D, Elbow \& Wrist, blue). The Control task was mainly associated with constant muscle torque against gravity (Fig. 4-4C, elbow \& Wrist, black).
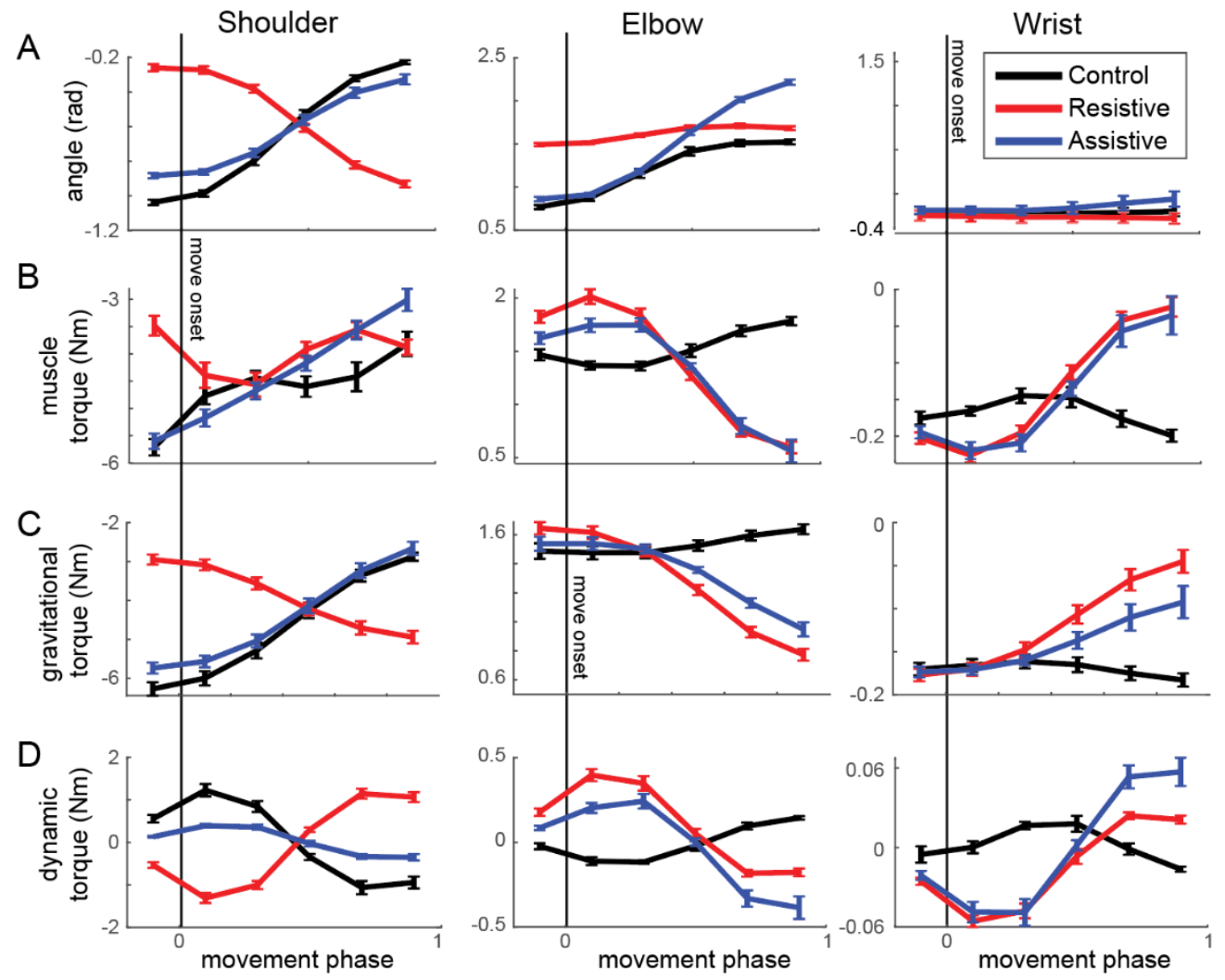

Figure 4-4: Movement kinematics and dynamics

Lines are mean trajectories; error bars are standard deviations across all participants. A. Joint angles calculated from motion-capture $B$. Muscle torques obtained from inverse simulations with the dynamic arm model. C. The gravity-related component of muscle torque obtained from comparing simulations with and without gravity. D. The dynamic component of muscle torque obtained from simulations without gravity. 

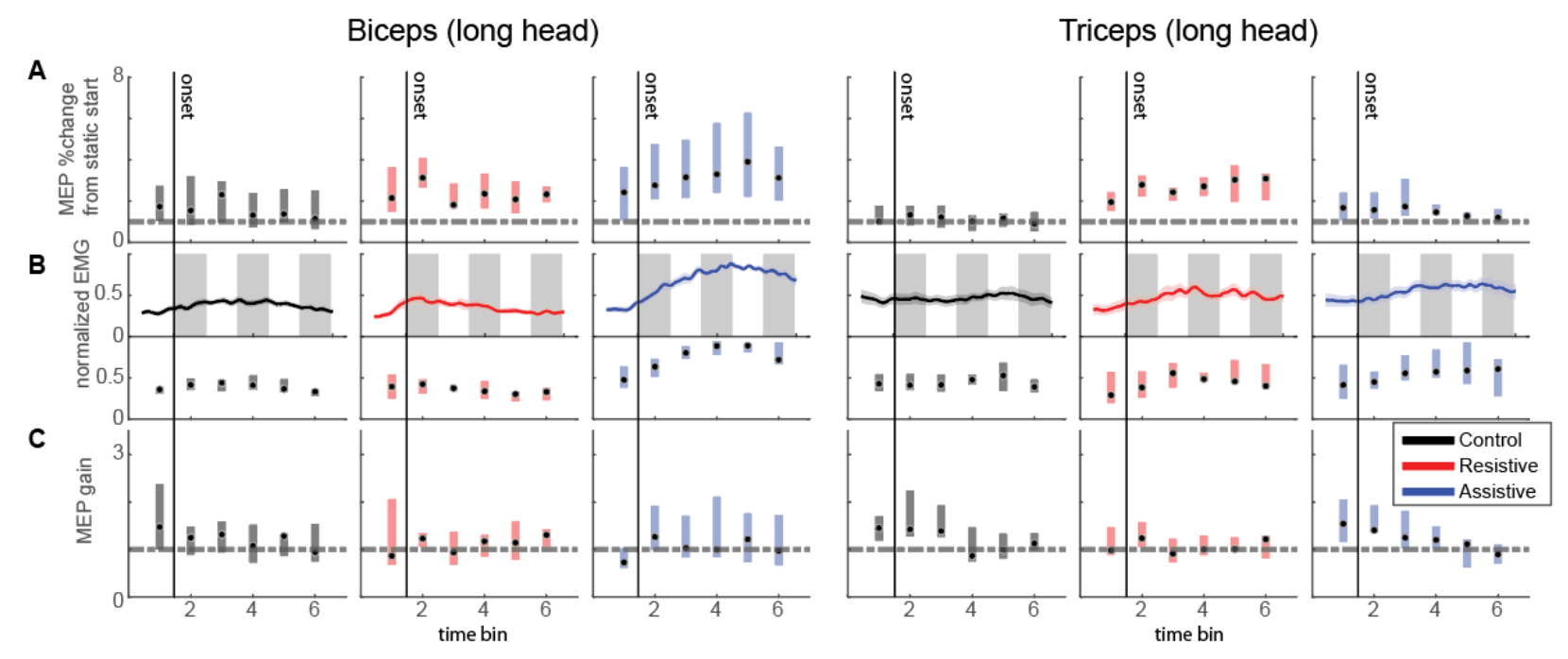

Figure 4-5: BicL \& TriLo corticospinal excitability during movement

MEP/EMG magnitudes binned relative to movement where bin 1 denotes MEPs preceding the kinematic onset of movement and bins 2-6 denote MEPs occurring during movement. Each bin size equals $20 \%$ of the total movement time. Black circles denote median magnitudes across participants and boxes denote interquartile range. A. Percent-change in MEP size relative to those recorded in each movement's starting posture. B. Muscle activity as recorded by EMG and the binned magnitudes corresponding to the binning procedure used for TMS responses. C. MEP gain defined as the ratio of MEP magnitudes to predicted MEP magnitudes based on background EMG.

\section{Corticospinal Excitability During Movement}

Motor evoked potentials recorded during movement displayed similar characteristics to static MEPs. MEPs were observed during movement with high frequency despite subthreshold stimulation and similarly had an average latency of $14.2 \pm 2.5 \mathrm{~ms}$. Conversely, MEPs during movement were more variable than those recorded in static postures. The median coefficient of variation was 0.50 during movement compared to 0.35 observed during static postures.

MEP magnitudes were temporally modulated during movement in a task dependent manner (Fig. 4-5A). The percent-change was calculated by dividing the magnitudes of MEPs recorded during movement with the MEP magnitude that was observed at the same initial posture in Session 1. Across all three movements, MEP magnitudes were greater than what was observed in static posture. In the Resistive task, MEPs were increased in the beginning of movement (bin 2). In the Assistive task, MEPs were 
increased during the middle of movement. The temporal profiles of MEPs reflected those of EMG suggesting that MEPs during movement may similarly follow the Boltzmann equation as observed in static postures (Fig. 4-5B). Therefore, we calculated MEP gain, as described in the Methods, to remove the contribution of EMG to MEP magnitude (Fig. 4-5C). We found that MEP gain values, which normalize the MEP to background EMG, were much closer to 1 . This suggests that MEP magnitude during movement may be explained largely by background EMG. We performed a linear regression between MEP magnitudes and EMG and found that median variance explained $\left(R^{2}\right)$ for BicL was 0.38 $\pm 0.27,0.26 \pm 0.28$, and $0.55 \pm 0.30$ for the Control, Resistive, and Assistive tasks respectively. . The $\mathrm{R}^{2}$ in the Assistive task, which also displayed the most co-activation of muscles, was greater than the Resistive task (paired t-test; p-value: 0.0087) (Fig. 4-6).

Similarly, the antagonist TriLo muscle showed task-dependent modulation of MEP magnitudes. However, this modulation was distinct from that observed in the BicL. For example, the percent-change in MEPs was largest in the Resistive task, in contrast to the BicL which showed the largest increase in the Assistive task, while the Control task showed no change. This difference was expected since background EMG for these two muscles also differs within tasks. Interestingly, MEP gain was greater than 1 in the beginning of both the Control and Assistive tasks, suggesting that some modulation of MEP magnitudes may be occurring independently of background EMG. 




Figure 4-6: BicL MEP variance explained by EMG

A linear regression was performed between integrated MEP and EMG magnitudes. The distribution of $R^{2}$ values across participants is shown for the Control (black), Resistive (red), and Assistive (blue) movements. 
A

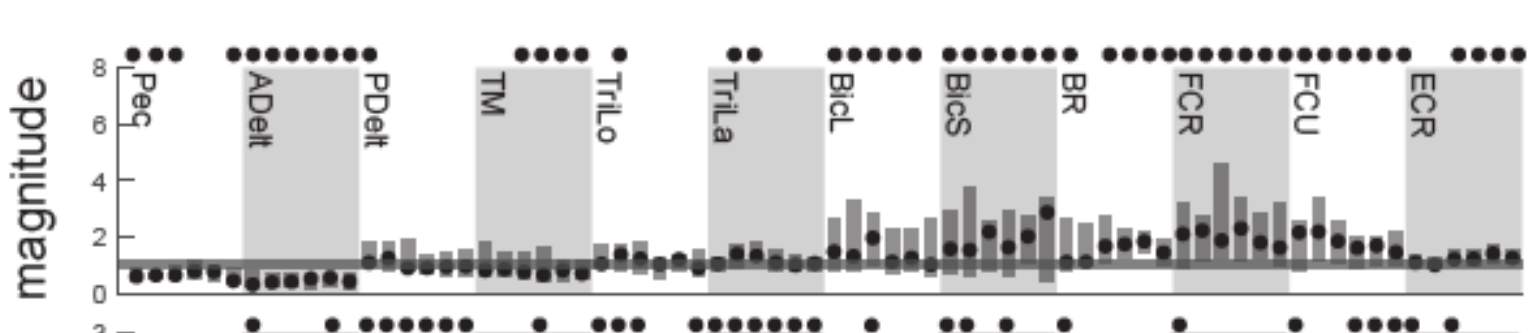

B
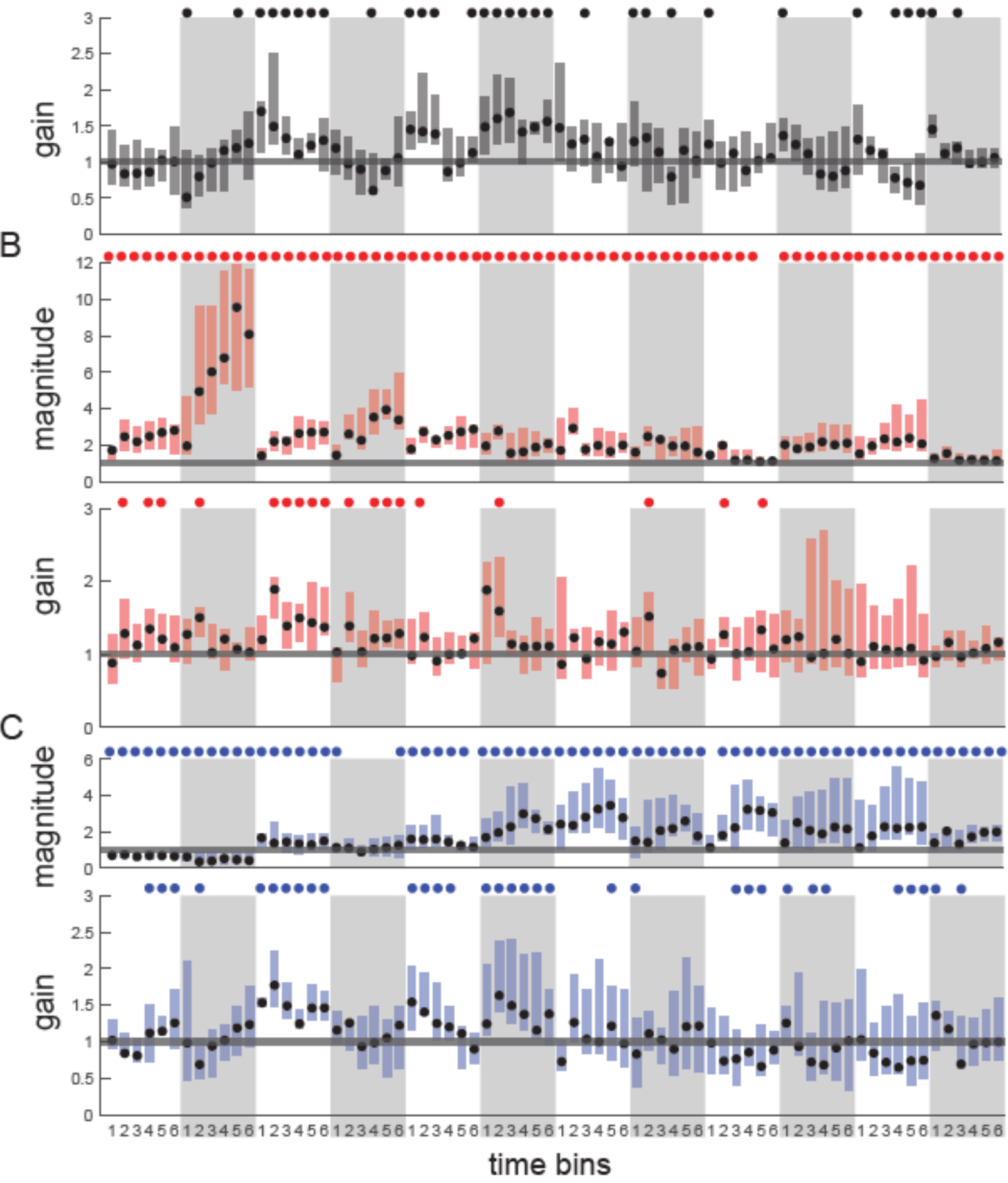

Figure 4-7: Multi-muscle MEP magnitude and gain 
MEP magnitude and gain across all recorded muscles for the control (black), resistive (red), and assistive (blue) movements. Median values across subjects are denoted with dots, while the bars denote interquartile range. MEP magnitudes which are statistically different from the initial static posture and MEP gains which differ from a value of 1 are denoted with dots.

Multi-muscle Corticospinal Excitability

Although our stimulation location targeted the biceps muscle, we observed MEPs in all recorded muscles. MEPs recorded in these muscles were also temporally modulated in a task-dependent manner similar to what was observed in the biceps and triceps (Fig. 4-7). Once again, these magnitudes largely reflected the activity of each muscle. For example, shoulder flexors (Pec \& AD) displayed increased MEP magnitude during the Resistive task which required flexion of the shoulder against gravity. Conversely, in both the Control and Assistive tasks which required shoulder extension, MEP magnitudes were decreased compared to the static start posture. Unsurprisingly changes in MEP magnitudes were prominent across all muscles. MEP gain also demonstrated modulation that was independent of muscle activity (i.e. gains not equal to 1), although this modulation occurred less frequently than those observed in MEP magnitudes.

\section{The Role of Limb Dynamics in Corticospinal Excitability}

Previously, it was shown that MEP gain correlated with resistive interaction torques (Gritsenko et al., 2011c). Our results show that while MEP magnitude is largely explained by background EMG, MEP gain are also modulated independently from background EMG. We used a generalized linear model to test whether changes in corticospinal excitability reflected not only EMG but also limb kinematics and/or dynamics. We used EMG, joint angles, muscle torques, and their components as predictors of either MEP magnitude or MEP gain across all subjects and muscles. The stepwise regression found EMG (slope: 0.32; SE: 0.01; t-stat: 23.5; p<0.001) and dynamic torques (slope: -0.17 ; SE: 0.03; t-stat: $-6.8 ; p<0.001)$ to be significant predictors of MEP magnitude along with the interaction of kinematics/gravity torque (slope: -0.15 ; SE: 0.03; t-stat: -4.7 ; $<<0.001$ ) and muscle torque/dynamic torque (slope: 0.13; SE: 0.04; t-stat: 3.6; p<0.001). The adjusted $\mathrm{R}^{2}$ was 0.23 . As can be seen from the slope estimates, EMG was unsurprisingly the strongest predictor of MEP magnitude. Conversely, when the same procedure was used 
to predict MEP gain instead of magnitude, EMG alone was found to be a significant predictor of MEP gain (slope: -0.17 ; SE: 0.03 ; t-stat: $-5.0 ; p<0.001$ ) along with the interaction between EMG/gravity torque (slope: 0.19; SE: 0.06; t-stat: 3.3; $p=0.0011$ ), suggesting that at higher levels of EMG, MEP magnitude is also correlated with gravity torques.

\section{Discussion}

Here we have taken advantage of Newtonian physics to design unconstrained reaching tasks in VR and precisely manipulate the passive forces acting on the human arm. During these tasks, we probed the gain of the corticospinal contribution to the compensation for these forces. We observed active corticospinal control of all recorded muscles, as evidenced by the presence of MEPs in all muscles during stimulation below resting motor threshold. We further found that during movement the corticospinal excitability was modulated largely proportionally to motoneuronal excitability with on average the same coefficient of proportionality as that during posture maintenance (Fig. 4-5). MEP variance explained by EMG did not differ between tasks (Fig. 4-6). The gain of corticospinal excitability during movement did not strongly correlate with limb dynamics. MEP gain was modulated in different tasks independent of muscle activity and correlated both inversely to $E M G$ and directly with the EMG:gravity torque interaction. Altogether we interpret these

results as evidence that corticospinal pathways are being actively modulated to compensate for distinct limb dynamics.

Here we used a novel TMS paradigm to investigate the temporal modulation of corticospinal excitability inspired by previous work with TMS during planar movements with unchanging gravity (Gritsenko et al., 2011c). We applied TMS over the primary motor cortex at $90 \%$ of resting motor threshold with a $45^{\circ}$ posterior-anterior coil orientation to preferentially activate pyramidal tract neurons (Kaneko et al., 1996; Nakamura et al., 1996; Di Lazzaro et al., 1998) and generate responses proportional to cortical activity (Baker et al., 1995; Carson et al., 1999; MacKinnon and Rothwell, 2000a; Di Lazzaro et al., 2003; Cros et al., 2007). The corticospinal tract and other descending tracts converge on the motoneurons, the final common pathway. Therefore, TMS responses are also modulated by the excitability of those motoneurons (MacKinnon and Rothwell, 2000a; 
Darling et al., 2006; Taylor, 2006; Groppa et al., 2012). Here we too observed that the TMS responses were linearly modulated by the motoneuronal excitability estimated from EMG during static postures (Table 3). This linear relationship is consistent with previous work by Darling et al. (Darling et al., 2006). Alternatively, Tazoe et al. (Tazoe et al., 2007) found that increases in MEP amplitude become saturated at higher intensities of muscle activity (> 60\% MVC). However, in our study participants were asked to complete gross reaching tasks with no additional load than the weight of the arm, which made it unlikely that any movement exceeded this linear range. Moreover, this simple linear relationship between MEP and EMG captured a lot of variance in TMS responses during movement (see Results and changes between MEP amplitude and gain in Fig. 4-6 and 4-7). The MEPs proportional to EMG with a constant coefficient can be interpreted as evidence for a tonic excitatory drive from corticospinal projections that rely on other sub-cortical and/or spinal pathways to shape motoneuronal excitability, not unlike a tonic speed command to drive spinal Central Pattern Generator, which produces phasic motoneuronal excitability patterns during locomotion (Yakovenko et al., 2018).

The putative tonic excitatory drive, however, explained only partially the modulation of corticospinal excitability during movement. Changes in corticospinal excitability during movement were largely explained by background EMG which was consistent with observations during isometric contractions (Darling et al., 2006). However, MEP gain, which controlled for motoneuronal excitability, was also modulated in a task dependent manner in some muscles. Characteristics of MEPs correspond to neurophysiological properties of the corticospinal pathways being stimulated (Di Lazzaro et al., 2004). For example, subthreshold stimulation using TMS has been shown to be an effective technique for probing changes in corticospinal excitability (Pascual-Leone et al., 1994; Chen et al., 1998; MacKinnon and Rothwell, 2000b; MacKinnon et al., 2004). The role of the primary motor cortex in the formation of the motor commands is still poorly understood, but single-cell recordings of pyramidal neurons have been correlated with kinematic and dynamic variables of movement including direction (Georgopoulos et al., 1986; Georgopoulos and Carpenter, 2015), force (Evarts, 1968), and velocity (Moran and Schwartz, 1999). TMS has previously been used to probe the modality of corticospinal pathways during movement and found that MEP gain correlated with resistive interaction 
torques (Gritsenko et al., 2011c). While our generalized linear model found a significant relationship with gravity component of active torques and MEP gain that was conditional on EMG. Furthermore, MEP magnitude was correlated with the dynamic torques although to a lesser extent than EMG. It is noteworthy that the movements performed in this study were unconstrained with changing gravity load on the joints, while the previous findings used an exoskeleton to constrain the reaching tasks on a plane with unchanging gravity load. The additional weight of the exoskeleton may exaggerate passive interaction torques causing an increased effect which was not observed in unconstrained movements. Conversely, the presence of changing gravity moments about the joints can explain the novel result observed here. Overall, we observed that MEP gain correlated weakly with kinematic and dynamic variables, which suggests that these variables are not the directly controlled parameters within the central nervous system. In contrast, the clear differences in MEP gains observed between tasks in individual muscles suggest that the corticospinal excitability is modulated during movement with different passive limb dynamics based on the functional roles of individual muscles. This modulation could be the product of broader cell population dynamics that represent neuromechanical variables in a different state space (Pandarinath et al., 2018).

\section{Chapter 5 - Discussion and Future Directions}

This dissertation explores the important role of neuromechanics in the arm motor control strategy. We applied a "bottom-up" approach which described and characterized the complex mechanics of the arm using biomechanical models and inferred neural control based upon electrophysiological recordings in humans. Specifically, we created 1) a musculoskeletal model of the upper limb which described muscle path geometry across a wide range of motion and 2) a mechanical model of the limb capable of approximating inverse dynamics of the shoulder, elbow, and wrist. In Chapter 2, we analyzed the mechanical co-dependencies of muscle lengths which constrain the neural control problem. In Chapter 3, we implemented this musculoskeletal model with a mathematical description of muscle spindle primary afferent firing frequency to quantify the activity of this afferent pathway during voluntary reaching tasks. The movements for these tasks were selected based upon their mechanical properties approximated from our inverse dynamics model. Finally, in Chapter 4 , we probed the corticospinal pathways using 
transcranial magnetic stimulation to investigate changes in the excitability of these pathways in the same dynamically distinct reaching tasks. This discussion will recapitulate the major findings of each chapter in the broader context of the motor control strategy and discuss future directions for this work.

\section{Musculoskeletal Model}

In Chapter 2, we analyzed the complex relationships of muscle lengths across a wide range of motion. We found that the high dimensionality of the musculoskeletal system contains a degree of mechanical coupling that could effectively constrain this system. The "motor redundancy" problem, formalized by Nikolai Berstein, observes that the mechanics of the musculoskeletal system has inherent redundancy, making it an underdefined control system (Bernstein, 1967).This feature raises questions as to how the nervous system can select and control this system in the presence of such redundancy. Our results provide a possible mechanism for reducing this dimensionality via mechanical constraints. An alternative, although possibly complementary, theory of dimensionality reduction is that of motor primitives or muscle synergy (Torres-Oviedo et al., 2006; Chvatal and Ting, 2013; Ting et al., 2015a). Muscle synergies describe the co-activation of muscles through some common neural input, which could also serve to reduce the dimensionality of the motor control problem (Bizzi et al., 1991; Giszter et al., 1993; Tresch et al., 1999; d'Avella et al., 2003b; Takei et al., 2017). Our results are consistent with previous locomotion studies that suggest that this dimensionality reduction can, at least in part, be achieved by the mechanical constraints inherent in the musculoskeletal system (Taga et al., 1991b; Full and Koditschek, 1999; Prochazka and Yakovenko, 2007b).

These results have important implications when considering the theory of muscle synergies as a control strategy. Traditionally, muscle synergies are extracted from EMG via signal decomposition techniques such as principal component analysis (PCA) or nonnegative matrix factorization (NNMF)(Torres-Oviedo et al., 2006; Chvatal and Ting, 2013; Ting et al., 2015b; Olesh et al., 2017c). These techniques compute orthogonal vectors which represent shared variance across the data matrix, in this case, EMG across multiple muscles. However, it may be difficult to determine if this lower dimensional space is due to kinematic/task constraints or common neural input (De Groote et al., 2014; 
Tagliabue et al., 2015). The presence of a common neural input is typically inferred 1) due to correlation between muscle synergies and decomposed neural recordings (Takei et al., 2017) or 2) due to complex movements being elicited from microstimulation of motor cortices (Graziano et al., 2002; Graziano and Aflalo, 2007). If we assume that muscle synergy does arise from a common neural input into multiple pools of spinal motoneurons, then it would follow that multiple muscles would receive similar activation profiles simultaneously. This common input could be advantageous when multiple muscles require similar recruitment. For example, when performing a whole-hand grasp, one could imagine a similar activation of finger flexors across all digits. However, if this common input is to generate a similar mechanical output, then there must be a similarity between the underlying musculoskeletal parameters, such as muscle lengths and moment arms. Our results demonstrate that finger flexors are mechanically coupled and change the same way across postures. As the finger digits are flexed, the muscle length shortens generating less force due to the force-length relationship. However, because muscle lengths are changing synchronously, a common input could still be appropriate to generate a similar force across all finger flexor muscles. Similarly, finger moment arms increase across all digits which once again could enable a common input to control. While our results cannot be said to explicitly provide evidence of this common input, the observed coupling does demonstrate that the musculoskeletal system is mechanically compatible with this low-dimensional input. Furthermore, our results suggest that musculoskeletal constraints must be considered when interpreting muscle activation patterns and their neural origin (De Groote et al., 2014).

Validation of muscle moment arms in this model is ongoing, but future work could examine whether the structural relationships in muscle lengths is conserved within muscle moment arms. The shoulder, in particular, presents an interesting study in complexity due to its high number of DOFs ( 3 rotation and 3 translational). The moment arm measurements of shoulder muscles in the literature suggest that a large number of muscles have both positive and negative moment arm values, i.e. the muscle's direction of action and functional role is posture-dependent. Once all moment arms of the shoulder have been validated, an analysis of the number of physiologically accurate zero crossings could provide insight into this complex joint. 


\section{Muscle Spindle Feedback}

In Chapter 3, we explored the contribution of the muscle spindle primary afferent pathway to muscle activity. Muscle spindles have long been associated with maintaining mechanical homeostasis but are also active during voluntary movement (Prochazka and Gorassini, 1998; Prochazka, 1999; Mileusnic et al., 2006). We simulated muscle spindle primary afferent activity using our musculoskeletal model to approximate muscle length profiles during reaching tasks. We found afferent firing frequency to be orthogonal to muscle activity and to be more closely related to the kinematics of each movement rather than the dynamics. We then modified the primary afferent model to simulate different fusimotor modulation, specifically modelling different 'fusimotor sets' (Prochazka et al., 1985; Prochazka, 1986) and $\alpha-y$ coupling (Granit, 1970; Hagbarth, 1993), but found that neither increased the congruency with EMG.

These results suggest two possibilities. The first possibility is that muscle spindles do not greatly contribute to motoneuron recruitment during voluntary movement. Although muscle spindle primary afferents will inevitably discharge during the lengthening of muscles during movement, muscle activity is instead driven by other neural circuitry (Yakovenko et al., 2004) that overrides and/or suppresses the incongruent afferent contribution to motoneuronal excitability. This interpretation would conflict with the spindle-centric equilibrium-point hypothesis which states that both movement and posture result from the balance of antagonist muscle spindle feedback. Additionally, the observation that la feedback is more consistent across movements than EMG suggests that muscle activity may instead be driven by more feedforward mechanisms than the muscle spindle feedback. The second possibility is that muscle spindles do contribute to motoneuron recruitment but require more complex fusimotor modulation than we modeled in our study. This limitation is important to note as our computational study cannot exclude this second possibility, nor can it "disprove" the equilibrium-point hypothesis. However, our findings do constrain the possible framework in which muscle spindles could drive muscle activity. Future work could inversely solve for this required theoretical fusimotor input by maximizing the EMG congruency to examine its features. Additionally, other spinal reflexes could be modeled and their activity integrated with muscle spindles, 
such as Golgi Tendon Organs, to examine whether muscle activity may instead result from some combination of these spinal circuits.

\section{Descending Corticospinal Contribution}

In Chapter 4, we probed the excitability of corticospinal pathways during dynamically distinct reaching tasks. We found evidence that the excitability of corticospinal pathways was increased during static postures suggesting that the primary motor cortex is involved in posture maintenance. During movement, we found that MEP gain was modulated in a task-dependent manner across multiple muscles. The differential modulation of MEPs during dynamically distinct tasks, independent from motoneuron activity, suggests that these dynamics are being actively compensated for by these descending pathways. However, this modulation correlated weakly with the kinematic and dynamic variables associated with these tasks. One possible explanation for this result may be that the descending corticospinal projections may represent limb dynamics in another state space which integrates these dynamics with other mechanical variables of movement to form a motor command(Pandarinath et al., 2018). This command is then integrated with primary afferent projections and spinal interneurons to form muscle activity patterns that can compensate for the complex mechanical interactions of musculoskeletal system.

Future work could explore whether the modulation of corticospinal excitability is impaired after neural injury, such as a cortical infarct. Stroke survivors have been shown to have an impaired ability to compensate for limb dynamics (Beer et al., 2000) and atypical muscle activation patterns (Ramos-Murguialday et al., 2015). If this modulation is, in fact, due to the compensation of limb dynamics, we would expect that individuals who have an impaired ability to compensate for these dynamics to have atypical modulation of MEPs.

\section{Conclusions}

In conclusion, my dissertation explored the representation and integration of limb dynamics/mechanics into the arm motor control strategy on the musculoskeletal, spinal, and cortical levels of the control hierarchy. The musculoskeletal system was found to contain inherent mechanical coupling constraints which may decrease the dimensionality 
of the control system. Muscle spindle activity was found to be largely orthogonal to muscle activity patterns and may instead serve primarily to supply information of the limb state via long-latency reflex pathways. Finally, the corticospinal tract was directly driving muscle contraction patterns in dynamically distinct reaching tasks, particularly when muscles were recruited to support the weight of the arm. Collectively, this hierarchical system provides a control structure which enables motor control despite the high complexity of the system and the variability of the external environment on which it acts. Future work will more comprehensively characterize these complex relationships with more physiologically accurate models and experimental data. Furthermore, it will lead to a better understanding of the necessary sensorimotor transformations that must occur at each level of this control hierarchy.

\section{References}

Ackland DC, Pak P, Richardson M, Pandy MG (2008) Moment arms of the muscles crossing the anatomical shoulder. Journal of Anatomy 213:383-390.

Angel MJ, Guertin P, Jiménez I, McCrea DA (1996) Group I extensor afferents evoke disynaptic EPSPs in cat hindlimb extensor motorneurones during fictive locomotion. J Physiol (Lond) 494 ( Pt 3):851-861.

Asatryan DG, Feldman AG (1965) Functional tuning of the nervous system with control of movements or maintenance of a steady posture: I. Mechanographic analysis of the work of the joint on execution of a postural task. Biophysics 10:925-935.

Aurbach M, Spicka J, Süß F, Dendorfer S (2020) Evaluation of musculoskeletal modelling parameters of the shoulder complex during humeral abduction above $90^{\circ}$. Journal of Biomechanics:109817.

Baker SN, Olivier E, Lemon RN (1995) Task-related variation in corticospinal output evoked by transcranial magnetic stimulation in the macaque monkey. The Journal of Physiology 488 ( Pt 3:795-801.

Bastian AJ, Martin TA, Keating JG, Thach WT (1996) Cerebellar ataxia: abnormal control of interaction torques across multiple joints. Journal of Neurophysiology 76:492-509.

Beer RF, Dewald JPA, Rymer WZ (2000) Deficits in the coordination of multijoint arm movements in patients with hemiparesis: evidence for disturbed control of limb dynamics. Exp Brain Res 131:305-309.

Benjamin EJ et al. (2017) Heart Disease and Stroke Statistics-2017 Update. Circulation 135:e146-e603. 
Benjamini Y, Krieger AM, Yekutieli D (2006) Adaptive linear step-up procedures that control the false discovery rate. Biometrika 93:491-507.

Bernstein N (1967) The co-ordination and regulation of movements. Journal of the Neurological Sciences Available at: https://www.jns-journal.com/article/0022-510X(68)901664/abstract [Accessed June 24, 2020].

Bestmann S, Krakauer JW (2015) The uses and interpretations of the motor-evoked potential for understanding behaviour. Experimental Brain Research 233:679-689.

Bizzi E, Mussa-Ivaldi FA, Giszter S (1991) Computations underlying the execution of movement: a biological perspective. Science 253:287-291.

Blum KP, Lamotte D'Incamps B, Zytnicki D, Ting LH (2017) Force encoding in muscle spindles during stretch of passive muscle Ayers J, ed. PLoS Comput Biol 13:e1005767.

Boots MT, Hardesty R, Sobinov A, Gritsenko V, Collinger JL, Fisher LE, Gaunt R, Yakovenko S (2020) Functional and Structural Moment Arm Validation for Musculoskeletal Models: A Study of the Human Forearm and Hand. bioRxiv:2020.05.29.124644.

Boyd IA (1985) The Muscle Spindle (Gladden MH, ed)., First Edition edition. New York, N.Y. : Houndmills, Basingstoke, Hampshire: Stockton Pr.

Brown IE, Loeb GE (2000) A Reductionist Approach to Creating and Using Neuromusculoskeletal Models. In: Biomechanics and Neural Control of Posture and Movement, pp 148-163. New York: Springer New York.

Brown P (1994) Pathophysiology of spasticity. Journal of Neurology, Neurosurgery \& Psychiatry 57:773-777.

Burdet E, Osu R, Franklin DW, Milner TE, Kawato M (2001) The central nervous system stabilizes unstable dynamics by learning optimal impedance. Nature 414:446-449.

Burke RE (1999) The use of state-dependent modulation of spinal reflexes as a tool to investigate the organization of spinal interneurons. Exp Brain Res 128:263-277.

Carson RG, Riek S, Bawa P (1999) Electromyographic activity, H-reflex modulation and corticospinal input to forearm motoneurones during active and passive rhythmic movements. Human movement science 18:307-343.

Chen R, Classen J, Gerloff C, Celnik P, Wassermann EM, Hallett M, Cohen LG (1997) Depression of motor cortex excitability by low-frequency transcranial magnetic stimulation. Neurology 48:1398-1403.

Chen R, Cros D, Curra A, Di Lazzaro V, Lefaucheur J-P, Magistris MR, Mills K, Rösler KM, Triggs WJ, Ugawa Y, Ziemann U (2008) The clinical diagnostic utility of transcranial magnetic stimulation: report of an IFCN committee. Clin Neurophysiol 119:504-532. 
Chen R, Yaseen Z, Cohen LG, Hallett M (1998) Time course of corticospinal excitability in reaction time and self-paced movements. Ann Neurol 44:317-325.

Chen WJ, Poppele RE (1978) Small-signal analysis of response of mammalian muscle spindles with fusimotor stimulation and a comparison with large-signal responses. Journal of Neurophysiology 41:15-27.

Cheung VCK, Piron L, Agostini M, Silvoni S, Turolla A, Bizzi E (2009) Stability of muscle synergies for voluntary actions after cortical stroke in humans. Proc Natl Acad Sci USA 106:19563-19568.

Cholewa J, Gorzkowska A, Nawrocka A, Cholewa J (2017) Quality of life of people with Parkinson's disease in the context of professional work and physiotherapy. Med Pr 68:725-734.

Chvatal S a, Ting LH (2013) Common muscle synergies for balance and walking. Frontiers in computational neuroscience 7:48.

Collins S (2005) Efficient Bipedal Robots Based on Passive-Dynamic Walkers. Science 307:1082-1085.

Cos I, Duque J, Cisek P (2014) Rapid prediction of biomechanical costs during action decisions. J Neurophysiol 112:1256-1266.

Crevecoeur F, Scott SH (2014) Beyond Muscles Stiffness: Importance of State-Estimation to Account for Very Fast Motor Corrections Valero-Cuevas FJ, ed. PLoS Comput Biol 10:e1003869.

Crevecoeur F, Thonnard J-L, Lefèvre P (2009) Optimal integration of gravity in trajectory planning of vertical pointing movements. J Neurophysiol 102:786-796.

Cros D, Soto O, Chiappa KH (2007) Transcranial magnetic stimulation during voluntary action: directional facilitation of outputs and relationships to force generation. 1185:103-116.

d'Avella A, Portone A, Fernandez L, Lacquaniti F (2006) Control of fast-reaching movements by muscle synergy combinations. J Neurosci 26:7791-7810.

d'Avella A, Saltiel P, Bizzi E (2003a) Combinations of muscle synergies in the construction of a natural motor behavior. Nat Neurosci 6:300-308.

d'Avella A, Saltiel P, Bizzi E (2003b) Combinations of muscle synergies in the construction of a natural motor behavior. Nat Neurosci 6:300-308.

Damm L, McIntyre J (2008) Physiological Basis of Limb-Impedance Modulation During Free and Constrained Movements. Journal of Neurophysiology 100:2577-2588.

Darainy M, Ostry DJ (2008) Muscle cocontraction following dynamics learning. Exp Brain Res 190:153-163. 
Darling WG, Wolf SL, Butler AJ (2006) Variability of motor potentials evoked by transcranial magnetic stimulation depends on muscle activation. Experimental Brain Research 174:376-385.

De Groote F, Jonkers I, Duysens J (2014) Task constraints and minimization of muscle effort result in a small number of muscle synergies during gait. Front Comput Neurosci 8:115.

De Luca CJ, Donald Gilmore L, Kuznetsov M, Roy SH (2010) Filtering the surface EMG signal: Movement artifact and baseline noise contamination. Journal of Biomechanics 43:15731579.

De Luca CJ, Hostage EC (2010) Relationship Between Firing Rate and Recruitment Threshold of Motoneurons in Voluntary Isometric Contractions. Journal of Neurophysiology 104:1034-1046.

De Serres SJ, Milner TE (1991) Wrist muscle activation patterns and stiffness associated with stable and unstable mechanical loads. Exp Brain Res 86:451-458.

Dekleva BM, Kording KP, Miller LE (2018) Single reach plans in dorsal premotor cortex during a two-target task. Nat Commun 9:3556.

Delp SL, Anderson FC, Arnold AS, Loan P, Habib A, John CT, Guendelman E, Thelen DG (2007) OpenSim: open-source software to create and analyze dynamic simulations of movement. IEEE Trans Biomed Eng 54:1940-1950.

Di Lazzaro V, Oliviero A, Pilato F, Mazzone P, Insola A, Ranieri F, Tonali PA (2003) Corticospinal volleys evoked by transcranial stimulation of the brain in conscious humans. Neurological research 25:143-150.

Di Lazzaro V, Oliviero A, Pilato F, Saturno E, Dileone M, Mazzone P, Insola A, Tonali PA, Rothwell JC (2004) The physiological basis of transcranial motor cortex stimulation in conscious humans. Clin Neurophysiol 115:255-266.

Di Lazzaro V, Oliviero A, Profice P, Saturno E, Pilato F, Insola A, Mazzone P, Tonali P, Rothwell JC (1998) Comparison of descending volleys evoked by transcranial magnetic and electric stimulation in conscious humans. Electroencephalography and clinical neurophysiology supplement 109:397-401.

Dideriksen JL, Negro F, Farina D (2015) The optimal neural strategy for a stable motor task requires a compromise between level of muscle cocontraction and synaptic gain of afferent feedback. Journal of Neurophysiology 114:1895-1911.

Drew T, Marigold DS (2015) Taking the next step: cortical contributions to the control of locomotion. Curr Opin Neurobiol 33:25-33.

Dunn OJ (1959) Estimation of the Medians for Dependent Variables. Ann Math Statist 30:192197. 
Duzgun Celik H, Cagliyan Turk A, Sahin F, Yilmaz F, Kuran B (2018) Comparison of disability and quality of life between patients with pediatric and adult onset paraplegia. J Spinal Cord Med 41:645-652.

Efron B, Tibshirani R (1993) An introduction to the bootstrap.

Efron B, Tibshirani RJ (1994) An Introduction to the Bootstrap, 1st ed. Chapman and Hall/CRC. Available at: https://www.crcpress.com/An-Introduction-to-the-Bootstrap/EfronTibshirani/p/book/9780412042317 [Accessed January 2, 2020].

Ellaway PH, Davey NJ, Maskill DW, Rawlinson SR, Lewis HS, Anissimova NP (1998) Variability in the amplitude of skeletal muscle responses to magnetic stimulation of the motor cortex in man. Electroencephalography and Clinical Neurophysiology Electromyography and Motor Control.

Evarts EV (1968) Relation of pyramidal tract activity to force exerted during voluntary movement. Journal of Neurophysiology 31:14-27.

Farina D, Negro F, Dideriksen JL (2014) The effective neural drive to muscles is the common synaptic input to motor neurons: Effective neural drive to muscles. J Physiol 592:34273441 .

Feldman AG (1966) Functional tuning of the nervous system with control of movement or maintenance of a steady posture. II. Controllable parameters of the muscle. Biophysics 11:565-578.

Fisher RA (1970) Statistical methods for research workers. Fourteenth Edition Revised, Enlarged 14th edition. Edinburgh: Oliver and Boyd.

Folgado J, Quental C, Ambrósio J, Monteiro J (2013) Multibody System of the Upper Limb Including a Reverse Shoulder Prosthesis. Journal of biomechanical engineering 135.

Fowlkes EB, Mallows CL (1983) A Method for Comparing Two Hierarchical Clusterings. Journal of the American Statistical Association 78:553-569.

Franklin DW, Liaw G, Milner TE, Osu R, Burdet E, Kawato M (2007) Endpoint Stiffness of the Arm Is Directionally Tuned to Instability in the Environment. Journal of Neuroscience 27:7705-7716.

Franklin DW, Osu R, Burdet E, Kawato M, Milner TE (2003) Adaptation to Stable and Unstable Dynamics Achieved By Combined Impedance Control and Inverse Dynamics Model. Journal of Neurophysiology 90:3270-3282.

Full RJ, Koditschek DE (1999) Templates and anchors: neuromechanical hypotheses of legged locomotion on land. J Exp Biol 202:3325-3332.

Gatev P, Thomas S, Kepple T, Hallett M (1999) Feedforward ankle strategy of balance during quiet stance in adults. The Journal of Physiology 514:915-928. 
Gendre T, Carle G, Mesrati F, Hubsch C, Mauras T, Roze E, Houot M, Degos B, Garcin B (2019) Quality of life in functional movement disorders is as altered as in organic movement disorders. J Psychosom Res 116:10-16.

Georgopoulos AP, Carpenter AF (2015) Coding of movements in the motor cortex. Current Opinion in Neurobiology 33:34-39.

Georgopoulos AP, Schwartz AB, Kettner RE (1986) Neuronal population coding of movement direction. Science (New York, NY) 233:1416-1419.

Ghika-Schmid F, Ghika J, Regli F, Bogousslavsky J (1997) Hyperkinetic movement disorders during and after acute stroke: The Lausanne Stroke Registry. Journal of the Neurological Sciences 146:109-116.

Gillard DM, Yakovenko S, Cameron T, Prochazka A (2000) Isometric muscle length-tension curves do not predict angle-torque curves of human wrist in continuous active movements. J Biomech 33:1341-1348.

Giszter SF, Mussa-Ivaldi FA, Bizzi E (1993) Convergent force fields organized in the frog's spinal cord. J Neurosci 13:467-491.

Granit R (1970) The basis of motor control;: Integrating the activity of muscles, alpha and gamma motoneurons and their leading control systems, First Edition edition. London, New York: Academic Press.

Graziano MSA, Aflalo TN (2007) Mapping behavioral repertoire onto the cortex. Neuron 56:239-251.

Graziano MSA, Taylor CSR, Moore T (2002) Complex Movements Induced by Microstimulation of Precentral Cortex. 34:841-851.

Grey MJ, Nielsen JB, Mazzaro N, Sinkjær T (2007) Positive force feedback in human walking. J Physiol 581:99-105.

Gribble PL, Mullin LI, Cothros N, Mattar A (2003) Role of Cocontraction in Arm Movement Accuracy. Journal of Neurophysiology 89:2396-2405.

Gribble PL, Ostry DJ (1999) Compensation for Interaction Torques During Single- and Multijoint Limb Movement. Journal of Neurophysiology 82:2310-2326.

Gritsenko V, Hardesty RL, Boots MT, Yakovenko S (2016) Biomechanical constraints underlying motor primitives derived from the musculoskeletal anatomy of the human arm. PLoS ONE 11:1-18.

Gritsenko V, Kalaska JF, Cisek P (2011a) Descending corticospinal control of intersegmental dynamics. The Journal of neuroscience : the official journal of the Society for Neuroscience 31:11968-79. 
Gritsenko V, Kalaska JF, Cisek P (2011b) Descending Corticospinal Control of Intersegmental Dynamics. Journal of Neuroscience 31:11968-11979.

Gritsenko V, Kalaska JF, Cisek P (2011c) Descending corticospinal control of intersegmental dynamics. The Journal of neuroscience : the official journal of the Society for Neuroscience 31:11968-11979.

Groppa S, Schlaak BH, Münchau A, Werner-Petroll N, Dünnweber J, Bäumer T, van Nuenen BFL, Siebner HR (2012) The human dorsal premotor cortex facilitates the excitability of ipsilateral primary motor cortex via a short latency cortico-cortical route. Human Brain Mapping 33:419-430.

Hagbarth KE (1993) Microneurography and applications to issues of motor control: Fifth Annual Stuart Reiner Memorial Lecture. Muscle Nerve 16:693-705.

Hardesty RL, Boots MT, Yakovenko S, Gritsenko V (2020) Computational evidence for nonlinear feedforward modulation of fusimotor drive to antagonistic co-contracting muscles. Scientific Reports 10:10625.

Harris C, Wolpert DM (1998) Signal-dependent noise determines motor planning. Nature 394:780-784.

He J, Levine WS, Loeb GE (1991) Feedback gains for correcting small perturbations to standing posture. IEEE Transactions on Automatic Control 36:322-332.

Hill AV (1938) The heat of shortening and the dynamic constants of muscle. Proceedings of the Royal Society of London:136-195.

Hirashima M, Kudo K, Ohtsuki T (2003) Utilization and Compensation of Interaction Torques During Ball-Throwing Movements. Journal of Neurophysiology 89:1784-1796.

Hoffer JA, Sugano N, Loeb GE, Marks WB, O’Donovan MJ, Pratt CA (1987) Cat hindlimb motoneurons during locomotion. II. Normal activity patterns. Journal of Neurophysiology 57:530-553.

Hoffman DS, Strick PL (1995) Effects of a primary motor cortex lesion on step-tracking movements of the wrist. Journal of Neurophysiology 73:891-895.

Hogan N, Bizzi E, Mussa-Ivaldi FA, Flash T (1987a) Controlling multijoint motor behavior. Exerc Sport Sci Rev 15:153-190.

Hogan N, Bizzi E, Mussa-Ivaldi FA, Flash T (1987b) Controlling multijoint motor behavior. Exerc Sport Sci Rev 15:153-190.

Hollerbach MJ, Flash T (1982) Dynamic interactions between limb segments during planar arm movement. Biol Cybern 44:67-77. 
Hongo T, Jankowska E, Lundberg A (1966) Convergence of excitatory and inhibitory action on interneurones in the lumbosacral cord. Exp Brain Res 1:338-358.

Howard IS, Ingram JN, Körding KP, Wolpert DM (2009) Statistics of natural movements are reflected in motor errors. J Neurophysiol 102:1902-1910.

Ingram JN, Körding KP, Howard IS, Wolpert DM (2008) The statistics of natural hand movements. Exp Brain Res 188:223-236.

Jeannerod M, Paulignan Y, Weiss P (1998) Grasping an object: one movement, several components. Novartis Found Symp 218:5-16; discussion 16-20.

Joyce GC, Rack PM (1969) Isotonic lengthening and shortening movements of cat soleus muscle. J Physiol (Lond) 204:475-491.

Kagaya K, Patek SN (2016) Feed-forward motor control of ultrafast, ballistic movements. Journal of Experimental Biology 219:319-333.

Kakuda N, Miwa T, Nagaoka M (1998) Coupling between single muscle spindle afferent and EMG in human wrist extensor muscles: physiological evidence of skeletofusimotor (beta) innervation. Electroencephalography and Clinical Neurophysiology/Electromyography and Motor Control 109:360-363.

Kaneko K, Kawai S, Fuchigami Y, Morita H, Ofuji A (1996) The effect of current direction induced by transcranial magnetic stimulation on the corticospinal excitability in human brain. Electroencephalography and clinical neurophysiology supplement 101:478-482.

Kawato M (1999) Internal models for motor control and trajectory planning. Curr Opin Neurobiol 9:718-727.

Kawato M, Furukawa K, Suzuki R (1987) A hierarchical neural-network model for control and learning of voluntary movement. Biol Cybern 57:169-185.

Kawato M, Samejima K (2007) Efficient reinforcement learning: computational theories, neuroscience and robotics. Curr Opin Neurobiol 17:205-212.

Kelly KM, Borstad AL, Kline D, Gauthier LV (2018) Improved quality of life following constraint-induced movement therapy is associated with gains in arm use, but not motor improvement. Topics in Stroke Rehabilitation 25:467-474.

Kiers L, Cros D, Chiappa KH, Fang J (1993) Variability of motor potentials evoked by transcranial magnetic stimulation. Electroencephalography and clinical Neurophysiology 89:415-423.

Krouchev N, Drew T (2013) Motor cortical regulation of sparse synergies provides a framework for the flexible control of precision walking. Front Comput Neurosci 7:83.

Kuo AD (2002) The Action of Two-joint Muscles : the Legacy of W . P . Lombard. 
Kurtzer I, Herter TM, Scott SH (2006) Nonuniform distribution of reach-related and torquerelated activity in upper arm muscles and neurons of primary motor cortex. Journal of Neurophysiology 96:3220-3230.

Kutch JJ, Kuo AD, Bloch AM, Rymer WZ (2008) Endpoint force fluctuations reveal flexible rather than synergistic patterns of muscle cooperation. J Neurophysiol 100:2455-2471.

Kutch JJ, Valero-Cuevas FJ (2012) Challenges and New Approaches to Proving the Existence of Muscle Synergies of Neural Origin Diedrichsen J, ed. PLoS Comput Biol 8:e1002434.

Lacquaniti F, Maioli C (1989) The role of preparation in tuning anticipatory and reflex responses during catching. J Neurosci 9:134-148.

Latash M (2008) Evolution of Motor Control: From Reflexes and Motor Programs to the Equilibrium-Point Hypothesis. Journal of Human Kinetics 19:3-24.

Latash ML, Scholz JP, Schöner G (2002) Motor control strategies revealed in the structure of motor variability. Exerc Sport Sci Rev 30:26-31.

Lawrence DG, Kuypers HG (1968) The functional organization of the motor system in the monkey. I. The effects of bilateral pyramidal lesions. Brain : a journal of neurology 91:114.

Leclere NX, Sarlegna FR, Coello Y, Bourdin C (2019) Sensori-motor adaptation to novel limb dynamics influences the representation of peripersonal space. Neuropsychologia 131:193-204.

Lesser RP, Arroyo S, Crone N, Gordon B (1998) Motor and sensory mapping of the frontal and occipital lobes. Epilepsia 39 Suppl 4:S69-80.

Lewis GN, Signal N, Taylor D (2014) Reliability of lower limb motor evoked potentials in stroke and healthy populations: How many responses are needed? Clinical Neurophysiology 125:748-754.

Leyton ASF, Sherrington CS (1917) Observations on the Excitable Cortex of the Chimpanzee, Orang-Utan, and Gorilla. Quarterly Journal of Experimental Physiology 11:135-222.

Liddell EGT, Sherrington CS (1924) Reflexes in response to stretch (myotatic reflexes). Proceedings of the Royal Society of London Series B, Containing Papers of a Biological Character 96:212-242.

Lo Buono V, Corallo F, Bramanti P, Marino S (2017) Coping strategies and health-related quality of life after stroke. J Health Psychol 22:16-28.

Loeb GE, Brown IE, Cheng EJ (1999) A hierarchical foundation for models of sensorimotor control. Experimental Brain Research 126:1-18. 
Lombard WP (1903) The Action of Two-Joint Muscles. American Physical Education Review 8:141-145.

Lundberg A (1969) Convergence of excitatory and inhibitory action on interneurones in the spinal cord. UCLA Forum Med Sci 11:231-265.

Macefield VG, Knellwolf TP (2018) Functional properties of human muscle spindles. Journal of Neurophysiology 120:452-467.

MacKinnon CD, Rothwell JC (2000a) Time-varying changes in corticospinal excitability accompanying the triphasic EMG pattern in humans. The Journal of Physiology 528:633645.

MacKinnon CD, Rothwell JC (2000b) Time-varying changes in corticospinal excitability accompanying the triphasic EMG pattern in humans. The Journal of Physiology 528:633645.

MacKinnon CD, Velickovic M, Drafta C, Hesquijarosa A, Brin MF (2004) Corticospinal excitability accompanying ballistic wrist movements in primary dystonia. Movement Disorders 19:273-284.

Malik P, Jabakhanji N, Jones KE (2016) An Assessment of Six Muscle Spindle Models for Predicting Sensory Information during Human Wrist Movements. Front Comput Neurosci 9 Available at: http://journal.frontiersin.org/Article/10.3389/fncom.2015.00154/abstract [Accessed January 2,2020$]$.

Martinez-Martin P (2017) What is quality of life and how do we measure it? Relevance to Parkinson's disease and movement disorders. Mov Disord 32:382-392.

Matthews PBC (1959a) A study of certain factors influencing the stretch reflex of the decerebrate cat. The Journal of Physiology 147:547-564.

Matthews PBC (1959b) A study of certain factors influencing the stretch reflex of the decerebrate cat. The Journal of Physiology 147:547-564.

Mileusnic MP, Brown IE, Lan N, Loeb GE (2006) Mathematical Models of Proprioceptors. I. Control and Transduction in the Muscle Spindle. Journal of Neurophysiology 96:17721788.

Milner TE, Franklin DW (2005) Impedance control and internal model use during the initial stage of adaptation to novel dynamics in humans: Initial stage of motor adaptation. The Journal of Physiology 567:651-664.

Moran DW, Schwartz AB (1999) Motor Cortical Representation of Speed and Direction During Reaching. Journal of Neurophysiology 82:2676-2692. 
Nakamura H, Kitagawa H, Kawaguchi Y, Tsuji H (1996) Direct and indirect activation of human corticospinal neurons by transcranial magnetic and electrical stimulation. Neuroscience Letters 210:45-48.

Nishikawa K, Biewener AA, Aerts P, Ahn AN, Chiel HJ, Daley MA, Daniel TL, Full RJ, Hale ME, Hedrick TL, Lappin AK, Nichols TR, Quinn RD, Satterlie RA, Szymik B (2007a) Neuromechanics: an integrative approach for understanding motor control. Integrative and Comparative Biology 47:16-54.

Nishikawa K, Biewener AA, Aerts P, Ahn AN, Chiel HJ, Daley MA, Daniel TL, Full RJ, Hale ME, Hedrick TL, Lappin AK, Nichols TR, Quinn RD, Satterlie RA, Szymik B (2007b) Neuromechanics: an integrative approach for understanding motor control. Integrative and comparative biology 47:16-54.

Olesh EV, Pollard BS, Gritsenko V (2017a) Gravitational and Dynamic Components of Muscle Torque Underlie Tonic and Phasic Muscle Activity during Goal-Directed Reaching. Frontiers in human neuroscience 11:1-12.

Olesh EV, Pollard BS, Gritsenko V (2017b) Gravitational and Dynamic Components of Muscle Torque Underlie Tonic and Phasic Muscle Activity during Goal-Directed Reaching. Front Hum Neurosci 11:474.

Olesh E V, Pollard BS, Gritsenko V (2017c) Gravitational and Dynamic Components of Muscle Torque Underlie Tonic and Phasic Muscle Activity during Goal-Directed Reaching. Frontiers in human neuroscience 11:1-12.

Overduin SA, d'Avella A, Roh J, Carmena JM, Bizzi E (2015) Representation of Muscle Synergies in the Primate Brain. J Neurosci 35:12615-12624.

Pandarinath C, Ames KC, Russo AA, Farshchian A, Miller LE, Dyer EL, Kao JC (2018) Latent Factors and Dynamics in Motor Cortex and Their Application to Brain-Machine Interfaces. J Neurosci 38:9390-9401.

Pasalar S, Roitman AV, Durfee WK, Ebner TJ (2006) Force field effects on cerebellar Purkinje cell discharge with implications for internal models. Nat Neurosci 9:1404-1411.

Pascual-Leone A, Valls-Solé J, Brasil-Neto JP, Cohen LG, Hallett M (1994) Akinesia in Parkinson's disease. I. Shortening of simple reaction time with focal, single-pulse transcranial magnetic stimulation. Neurology 44:884-891.

Patla AE (1985) Some characteristics of EMG patterns during locomotion: implications for the locomotor control process. J Mot Behav 17:443-461.

Penfield W, Rasmussen T (1950a) The cerebral cortex of man: A Clinical Study of Localization of Function.

Penfield W, Rasmussen T (1950b) The cerebral cortex of man; a clinical study of localization of function. Oxford, England: Macmillan. 
Perez MA, Lundbye-Jensen J, Nielsen JB (2007) Task-Specific Depression of the Soleus HReflex After Cocontraction Training of Antagonistic Ankle Muscles. Journal of Neurophysiology 98:3677-3687.

Perreault EJ, Kirsch RF, Crago PE (2001) Effects of voluntary force generation on the elastic components of endpoint stiffness. Exp Brain Res 141:312-323.

Prentice SD, Drew T (2001) Contributions of the Reticulospinal System to the Postural Adjustments Occurring During Voluntary Gait Modifications. Journal of Neurophysiology 85:679-698.

Prochazka A (1986) Proprioception during voluntary movement. Can J Physiol Pharmacol 64:499-504.

Prochazka A (1999) Chapter 11 Quantifying Proprioception. In: Progress in Brain Research (Binder MD, ed), pp 133-142 Peripheral and Spinal Mechanisms in the Neural Control of Movement. Elsevier. Available at:

http://www.sciencedirect.com/science/article/pii/S0079612308628502 [Accessed January 2, 2020].

Prochazka A (2011) Proprioceptive Feedback and Movement Regulation. In: Comprehensive Physiology (Terjung R, ed), pp cp120103. Hoboken, NJ, USA: John Wiley \& Sons, Inc. Available at: http://doi.wiley.com/10.1002/cphy.cp120103 [Accessed December 5, 2019].

Prochazka A, Gorassini M (1998) Models of ensemble firing of muscle spindle afferents recorded during normal locomotion in cats. The Journal of Physiology 507:277-291.

Prochazka A, Hulliger M, Zangger P (1985) "Fusimotor set": new evidence for a-independent control of y-motoneurones during movement in the awake cat. Brain Research 339:136140 .

Prochazka A, Yakovenko S (2007a) The neuromechanical tuning hypothesis. Progress in brain research 165:255-265.

Prochazka A, Yakovenko S (2007b) The neuromechanical tuning hypothesis. In: Progress in Brain Research (Cisek P, Drew T, Kalaska JF, eds), pp 255-265 Computational Neuroscience: Theoretical Insights into Brain Function. Elsevier. Available at: http://www.sciencedirect.com/science/article/pii/S0079612306650164 [Accessed January 9, 2020].

Prochazka A, Yakovenko S (2007c) The neuromechanical tuning hypothesis. Progress in brain research 165:255-265.

Pruszynski JA, Kurtzer I, Lillicrap TP, Scott SH (2009) Temporal Evolution of "Automatic Gain-Scaling.” Journal of Neurophysiology 102:992-1003.

Pruszynski JA, Kurtzer I, Nashed JY, Omrani M, Brouwer B, Scott SH (2011) Primary motor cortex underlies multi-joint integration for fast feedback control. Nature 478:387-390. 
Quental C, Folgado J, Ambrósio J, Monteiro J (2012) A multibody biomechanical model of the upper limb including the shoulder girdle. Multibody Syst Dyn 28:83-108.

Rack PM, Westbury DR (1974) The short range stiffness of active mammalian muscle and its effect on mechanical properties. J Physiol (Lond) 240:331-350.

Ramos-Lima MJM, Brasileiro I de C, de Lima TL, Braga-Neto P (2018) Quality of life after stroke: impact of clinical and sociodemographic factors. Clinics (Sao Paulo) 73 Available at: https://www.ncbi.nlm.nih.gov/pmc/articles/PMC6152181/ [Accessed June 24, 2020].

Ramos-Murguialday A, García-Cossio E, Walter A, Cho W, Broetz D, Bogdan M, Cohen LG, Birbaumer N (2015) Decoding upper limb residual muscle activity in severe chronic stroke. Ann Clin Transl Neurol 2:1-11.

Rana M, Yani MS, Asavasopon S, Fisher BE, Kutch JJ (2015) Brain Connectivity Associated with Muscle Synergies in Humans. J Neurosci 35:14708-14716.

Rancourt D, Hogan N (2001) Stability in force-production tasks. Journal of Motor Behavior.

Richard A, Van Hamme A, Drevelle X, Golmard J-L, Meunier S, Welter M-L (2017)

Contribution of the supplementary motor area and the cerebellum to the anticipatory postural adjustments and execution phases of human gait initiation. Neuroscience 358:181-189.

Roberts LV, Stinear CM, Lewis GN, Byblow WD (2008) Task-Dependent Modulation of Propriospinal Inputs to Human Shoulder. Journal of Neurophysiology 100:2109-2114.

Robertson G, Caldwell G, Hamill J, Kamen G, Whittlesey S (2013a) Research Methods in Biomechanics, 2E. Human Kinetics.

Robertson GE, Caldwell GE, Hamill J, Kamen G, Whittlesey S (2013b) Research Methods in Biomechanics, Second edition. Champaign, Illinois: Human Kinetics, Inc.

Rothwell JC (1997) Techniques and mechanisms of action of transcranial stimulation of the human motor cortex. J Neurosci Methods 74:113-122.

Sartori M, Reggiani M, van den Bogert AJ, Lloyd DG (2012) Estimation of musculotendon kinematics in large musculoskeletal models using multidimensional B-splines. Journal of Biomechanics 45:595-601.

Saul KR, Hu X, Goehler CM, Vidt ME, Daly M, Velisar A, Murray WM (2015a) Benchmarking of dynamic simulation predictions in two software platforms using an upper limb musculoskeletal model. Computer Methods in Biomechanics and Biomedical Engineering 18:1445-1458.

Saul KR, Hu X, Goehler CM, Vidt ME, Daly M, Velisar A, Murray WM, Murry WM (2015b) Benchmarking of dynamic simulation predictions in two software platforms using an 
upper limb musculoskeletal model. Comut Methods Biomech Biomed Engin 18:14451458.

Schöner G, Kelso JA (1988) Dynamic pattern generation in behavioral and neural systems. Science (New York, NY) 239:1513-1520.

Seki K, Perlmutter SI, Fetz EE (2003) Sensory input to primate spinal cord is presynaptically inhibited during voluntary movement. Nat Neurosci 6:1309-1316.

Shadmehr R, Mussa Ivaldi F (1994) Adaptive representation of dynamics during learning of a motor task. Journal of Neuroscience 14:3208-3224.

Shadmehr R, Smith MA, Krakauer JW (2010) Error Correction, Sensory Prediction, and Adaptation in Motor Control. Annual Review of Neuroscience 33:89-108.

Shenoy KV, Sahani M, Churchland MM (2013) Cortical control of arm movements: a dynamical systems perspective. Annual Review of Neuroscience 36:337-359.

Sherrington CS (1909) Reciprocal innervation of antagonistic muscles. Fourteenth note. - On double reciprocal innervation. Proceedings of the Royal Society of London Series B, Containing Papers of a Biological Character 81:249-268.

Sherrington CS (1910) Flexion-reflex of the limb, crossed extension-reflex, and reflex stepping and standing. The Journal of Physiology 40:28-121.

Sobinov A, Boots M, Gritsenko V, Fisher LE, Gaunt RA, Yakovenko S (2019) Approximating complex musculoskeletal biomechanics using multidimensional autogenerating polynomials. bioRxiv:759878.

Stepniewska I, Fang P-CY, Kaas JH (2009) Organization of the posterior parietal cortex in galagos: I. Functional zones identified by microstimulation. J Comp Neurol 517:765782.

Taga G, Yamaguchi Y, Shimizu H (1991a) Self-organized control of bipedal locomotion by neural oscillators in unpredictable environment. Biol Cybern 65:147-159.

Taga G, Yamaguchi Y, Shimizu H (1991b) Self-organized control of bipedal locomotion by neural oscillators in unpredictable environment. Biol Cybern 65:147-159.

Tagliabue M, Ciancio AL, Brochier T, Eskiizmirliler S, Maier MA (2015) Differences between kinematic synergies and muscle synergies during two-digit grasping. Front Hum Neurosci 9 Available at:

http://www.frontiersin.org/Human_Neuroscience/10.3389/fnhum.2015.00165/abstract [Accessed December 5, 2019].

Takei T, Confais J, Tomatsu S, Oya T, Seki K (2017) Neural basis for hand muscle synergies in the primate spinal cord. Proc Natl Acad Sci USA 114:8643-8648. 
Talkington WJ, Pollard BS, Olesh E V, Gritsenko V (2015) Multifunctional Setup for Studying Human Motor Control Using Transcranial Magnetic Stimulation, Electromyography, Motion Capture, and Virtual Reality. Journal of visualized experiments : JoVE:e52906.

Taylor J (2006) Stimulation at the cervicomedullary junction in human subjects. Journal of Electromyography and Kinesiology 16:215-223.

Tazoe T, Sakamoto M, Nakajima T, Endoh T, Komiyama T (2007) Effects of remote muscle contraction on transcranial magnetic stimulation-induced motor evoked potentials and silent periods in humans. Clinical Neurophysiology 118:1204-1212.

Tee KP, Franklin DW, Kawato M, Milner TE, Burdet E (2010) Concurrent adaptation of force and impedance in the redundant muscle system. Biol Cybern 102:31-44.

Terao Y, Ugawa Y, Uesaka Y, Hanajima R, Gemba-Shimizu K, Ohki Y, Kanazawa I (1995) Input-output organization in the hand area of the human motor cortex. Electroencephalogr Clin Neurophysiol 97:375-381.

Thelen DG, Anderson FC (2006) Using computed muscle control to generate forward dynamic simulations of human walking from experimental data. J Biomech 39:1107-1115.

Thickbroom GW, Byrnes ML, Mastaglia FL (1999) A model of the effect of MEP amplitude variation on the accuracy of TMS mapping. Clinical Neurophysiology.

Thoroughman KA, Shadmehr R (1999) Electromyographic Correlates of Learning an Internal Model of Reaching Movements. J Neurosci 19:8573-8588.

Ting LH, Chiel HJ, Trumbower RD, Allen JL, McKay JL, Hackney ME, Kesar TM (2015a) Neuromechanical Principles Underlying Movement Modularity and Their Implications for Rehabilitation. Neuron 86:38-54.

Ting LH, Chiel HJ, Trumbower RD, Allen JL, McKay JL, Hackney ME, Kesar TM (2015b) Neuromechanical principles underlying movement modularity and their implications for rehabilitation. Neuron 86:38-54.

Torres-Oviedo G, Macpherson JM, Ting LH (2006) Muscle synergy organization is robust across a variety of postural perturbations. J Neurophysiol 96:1530-1546.

Traversa R, Cicinelli P, Bassi A, Rossini PM, Bernardi G (1997) Mapping of motor cortical reorganization after stroke. A brain stimulation study with focal magnetic pulses. Stroke 28:110-117.

Tresch MC, Cheung VCK, d'Avella A (2006) Matrix factorization algorithms for the identification of muscle synergies: evaluation on simulated and experimental data sets. $\mathrm{J}$ Neurophysiol 95:2199-2212.

Tresch MC, Jarc A (2009) The case for and against muscle synergies. Current Opinion in Neurobiology 19:601-607. 
Tresch MC, Saltiel P, Bizzi E (1999) The construction of movement by the spinal cord. Nat Neurosci 2:162-167.

Valero-Cuevas FJ, Cohn BA, Yngvason HF, Lawrence EL (2015) Exploring the highdimensional structure of muscle redundancy via subject-specific and generic musculoskeletal models. Journal of biomechanics 48:2887-2896.

Wichmann T, Bergman H, Delong MR (2017) Basal ganglia, movement disorders and deep brain stimulation: advances made through non-human primate research. Journal of Neural Transmission Available at:

http://www.ncbi.nlm.nih.gov/pubmed/28601961\%0Ahttp://link.springer.com/10.1007/s0 0702-017-1736-5.

Winter DA (2009a) Biomechanics and Motor Control of Human Movement, 4th ed. Wiley. Available at: https://www.wiley.com/enus/Biomechanics + and + Motor + Control + of + Human + Movement $\% 2 C+4$ th + Edition-p9780470398180 [Accessed January 2, 2020].

Winter DA (2009b) Biomechanics and Motor Control of Human Movement, 4th ed. Hoboken, New Jersey: John Wiley \& Sons.

Winters JM, Stark L (1987) Muscle models: What is gained and what is lost by varying model complexity. Biol Cybern 55:403-420.

Wit LD, Theuns P, Dejaeger E, Devos S, Gantenbein AR, Kerckhofs E, Schuback B, Schupp W, Putman K (2017) Long-term impact of stroke on patients' health-related quality of life. Disability and Rehabilitation 39:1435-1440.

Wolpert DM, Kawato M (1998) Multiple paired forward and inverse models for motor control. Neural Netw 11:1317-1329.

Wolpert DM, Miall RC, Kawato M (1998) Internal models in the cerebellum. Trends in Cognitive Sciences 2:338-347.

Wong J, Wilson ET, Malfait N, Gribble PL (2009) Limb Stiffness Is Modulated With Spatial Accuracy Requirements During Movement in the Absence of Destabilizing Forces. Journal of Neurophysiology 101:1542-1549.

Yakovenko S (2011) A hierarchical perspective on rhythm generation for locomotor control. Progress in brain research 188:151-166.

Yakovenko S, Drew T (2015) Similar Motor Cortical Control Mechanisms for Precise Limb Control during Reaching and Locomotion. Journal of Neuroscience 35:14476-14490.

Yakovenko S, Gritsenko V, Prochazka A (2004) Contribution of stretch reflexes to locomotor control: a modeling study. Biological Cybernetics 90:146-155. 
Yakovenko S, Krouchev N, Drew T (2011) Sequential activation of motor cortical neurons contributes to intralimb coordination during reaching in the cat by modulating muscle synergies. J Neurophysiol 105:388-409.

Yakovenko S, Mushahwar V, VanderHorst V, Holstege G, Prochazka A (2002) Spatiotemporal activation of lumbosacral motoneurons in the locomotor step cycle. J Neurophysiol $87: 1542-1553$.

Yakovenko S, Sobinov A, Gritsenko V (2018) Analytical CPG model driven by limb velocity input generates accurate temporal locomotor dynamics. PeerJ 6.

Zajac FE (1989) Muscle and tendon: properties, models, scaling, and application to biomechanics and motor control. Crit Rev Biomed Eng 17:359-411.

Zehr EP, Balter JE, Ferris DP, Hundza SR, Loadman PM, Stoloff RH (2007) Neural regulation of rhythmic arm and leg movement is conserved across human locomotor tasks. J Physiol (Lond) 582:209-227. 